\title{
Generation of Novel Photochromic GFPs: Fluorescent Probes for RESOLFT-type Microscopy at Low Light Intensities
}

\author{
Dissertation \\ for the award of the degree \\ "Doctor rerum naturalium" \\ Division of Mathematics and Natural Sciences \\ of the Georg-August-Universität Göttingen
}

submitted by

Tim Grotjohann

from Hannover

Göttingen, 2012 


\section{Thesis committee members:}

- Prof. Dr. Stefan Jakobs (1st Referee)

Max Planck Institute for Biophysical Chemistry, Göttingen

Department of NanoBiophotonics

Mitochondrial Structure and Dynamics Group

- Prof. Dr. Kai Tittmann (2nd Referee)

Georg August University Göttingen

Albrecht von Haller Institute

Department of Bioanalytics

- Dr. Dieter Klopfenstein

Georg August University Göttingen

Third Institute of Physics

Department of Biophysics

Date of oral examination: $18^{\text {th }}$ April, 2012 


\section{Affidavit}

I hereby ensure that the presented thesis "Generation of novel photochromic GFPs: fluorescent probes for live cell RESOLFT-type microscopy at low light intensities" has been written independently and with no other sources and aids than quoted.

(Tim Grotjohann)

$10^{\text {th }}$ February 2012, Göttingen 
I.

II. Introduction

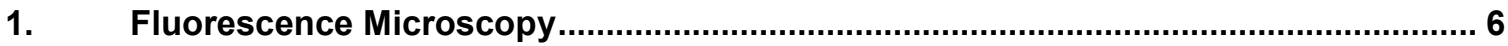

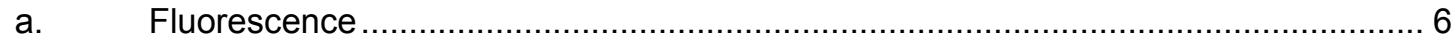

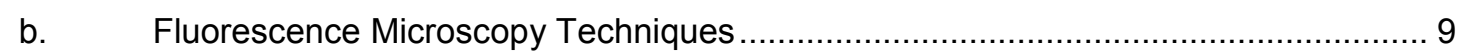

c. The Diffraction Barrier and Super-Resolution Microscopy .............................. 11

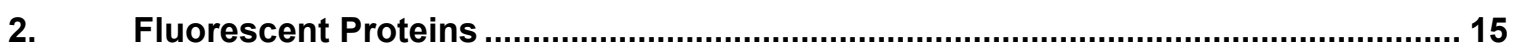

a. The Overall Structure of Fluorescent Proteins ............................................ 16

b. $\quad$ The Chromophore and Its Environment - Maturation, Conformation, Protonation ... 17

c. Tuning the Spectral Properties of Fluorescence Proteins................................ 22

d. Optimizing Fluorescent Protein Characteristics for Specific Needs ...................... 25

e. Photoactivatable and Photoconvertable Fluorescent Proteins .............................. 26

f. Reversibly Switchable Fluorescent Proteins ............................................ 29

III. Aims ........................................................................... 34

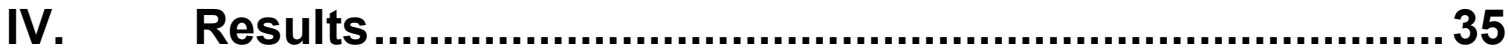

1. Generation and Establishment of a Screening System to Study RSFP Characteristics ................................................................................................... 35

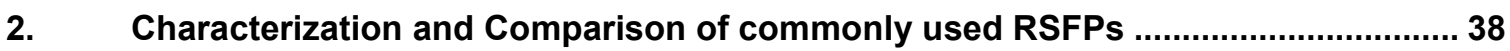

3. Reversibly Switchable Enhanced Green Fluorescent Protein (rsEGFP) ................ 40

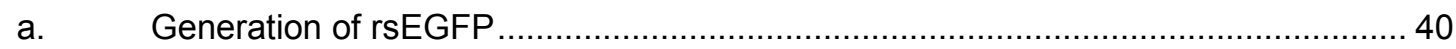

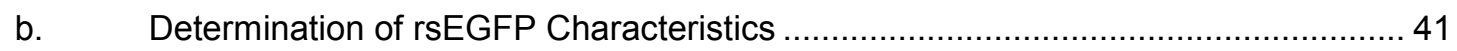

c. Using rsEGFP as a Rewritable Data Storage Medium ................................. 47

d. $\quad$ Establishing a RESOLFT-type Microscopy Approach Using rsEGFP ..................... 49

e. Establishing a Diffraction-Unlimited all Optical Data Storage Approach................. 53

4. Reversibly Switchable Enhanced Green Fluorescent Protein 2 (rsEGFP2) ............ 55

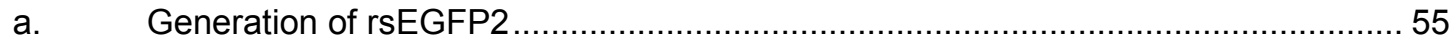

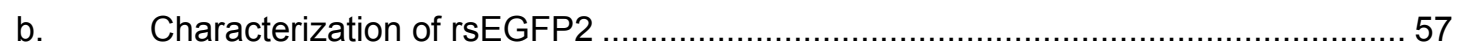

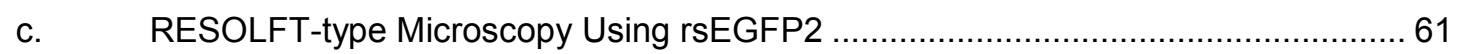


V. Discussion...............................................................6 66

1. A Comparison of Screening Approaches Utilized for RSFP Generation ..................67

2. Introduction of Fast, Persisting, and Reversible Switching into EGFP ...................69

a. The Non-Linear Dependence of Light Intensity on RSFP Characteristics ................69 69

b. The Structural Basis of Switching in rsEGFP and rsEGFP2 …............................. 70

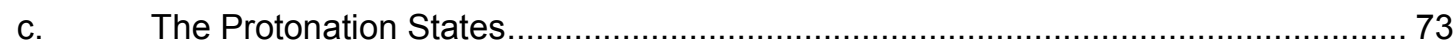

3. RESOLFT-type Microscopy Based on RSFPs ....................................................... 74

a. The Resolution of rsEGFP-RESOLFT Microscopy …......................................... 75

b. Super-Resolution Microscopy at Low Levels of Light Using rsEGFP-RESOLFT ..... 76

c. Increasing the Imaging Speed in RSFP-based RESOLFT Microscopy .................... 77

d. The Adaptability of the RESOLFT Approach to Different Experimental Needs ........ 79

4. All Optical Data Storage Approaches Using rsEGFP ............................................ 80

a. The Use of rsEGFP for Rewritable Data Storage Applications ............................... 80

b. $\quad$ rsEGFP Enables Diffraction-Unlimited All-Optical Writing ....................................... 82

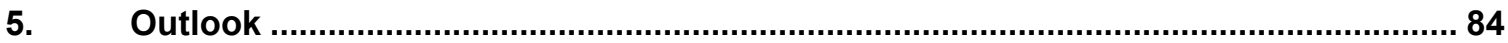

VI. Material \& Methods .................................................... 86

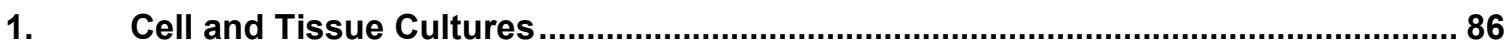

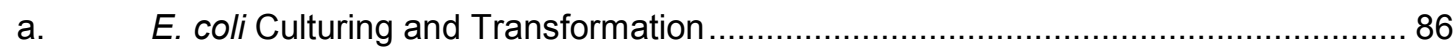

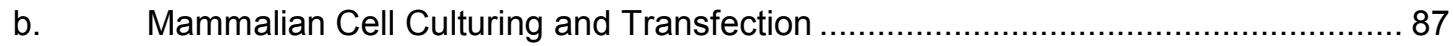

c. Preparation, Culturing, and Viral Transfection of Organtypic Hippocampal Slices... 87

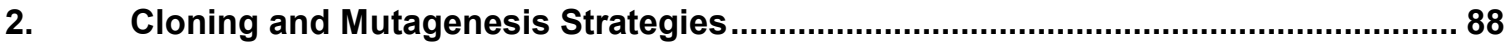

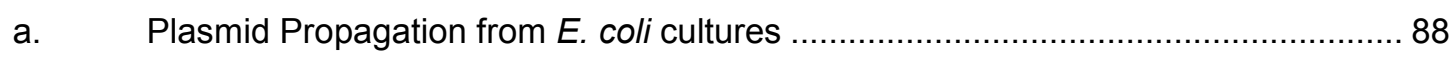

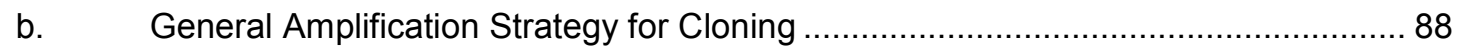

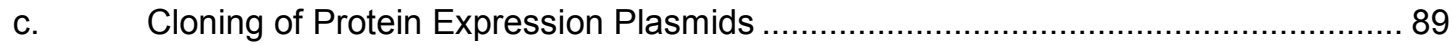

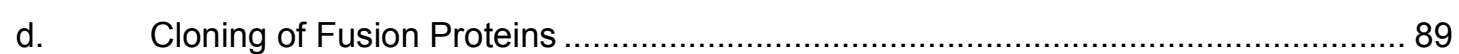

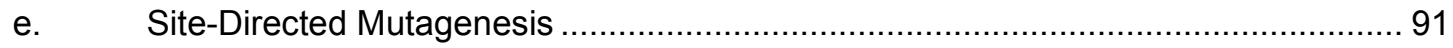

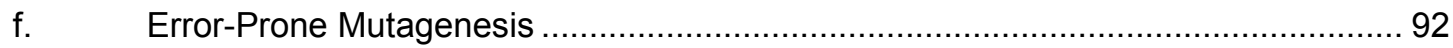

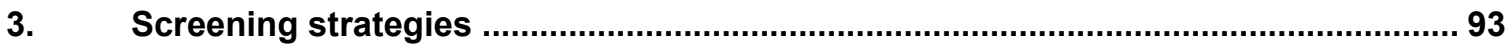

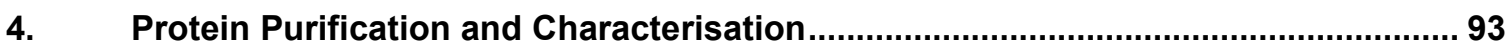

a. Protein Expression and Purification ..................................................................... 93

b. Determination of Absorption Spectra ……....................................................... 94

c. Determination of Fluorescence Excitation and Emission Spectra ............................ 94 
d. Calculation of Molecular Extinction Coefficients and Fluorescence Quantum Yield of

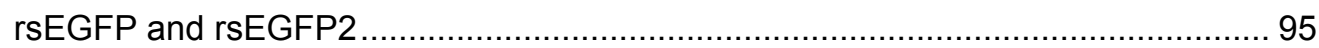

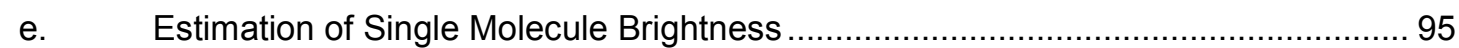

f. Determination of Switching and Bleaching Characteristics............................... 96

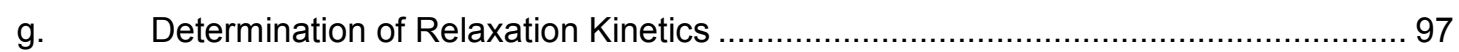

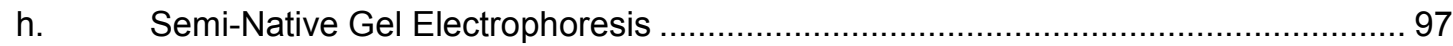

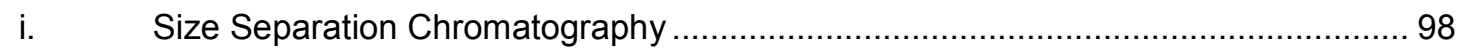

j. Determination of Chromophor Maturation Halftime .......................................... 98

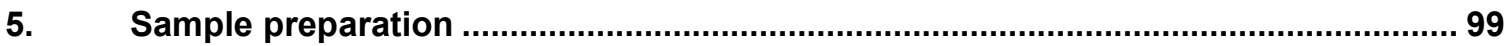

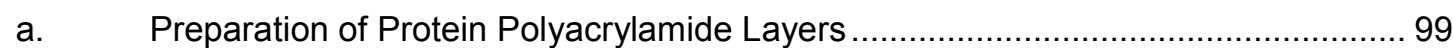

b. Preparing E. coli samples Expressing the rsEGFP-MreB Fusion Protein ............... 99

c. Preparing Mammalian Cell Samples for Microscopy ..................................... 100

d. Preparation of Organotypic Hippocampal Slice Cultures for Micorscopy .............. 100

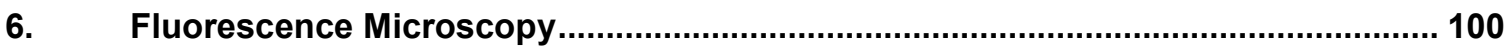

a. Confocal Laser Scanning Microscopy ......................................................... 100

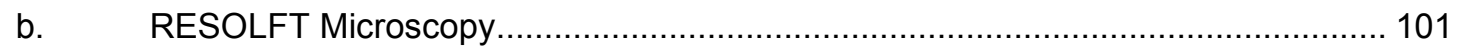

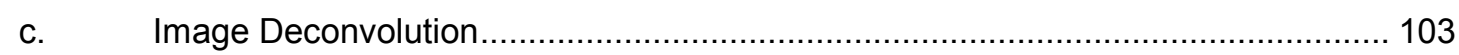

7. Data Storage Approaches .............................................................................. 104

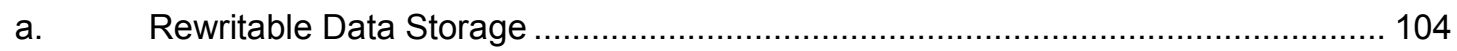

b. Diffraction Unlimited All-Optical Data Storage ................................................ 105

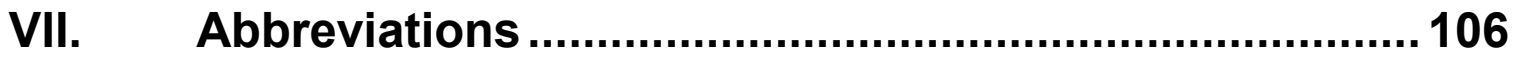

VIII. References ……......................................................... 108

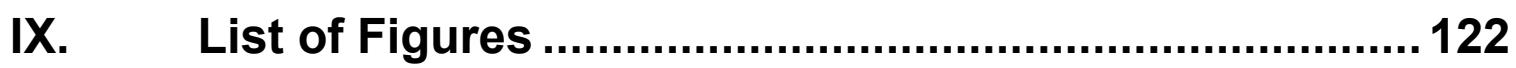

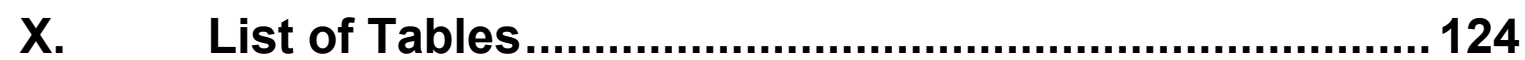

XI. Acknowledgement ....................................................125

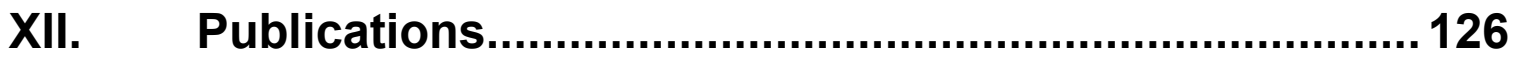

XIII. Curriculum vitae......................................................... 127 


\section{Summary}

The resolution of a conventional light microscope is limited by diffraction. Hence, light microscopy failed to discern objects that are closer together than half the wavelength of light. STED (STimulated Emission Depletion) microscopy overcame this diffraction resolution barrier for the first time by targeted switching of the fluorescence capability of fluorophores with intense light.

This work presents the generation of two novel reversibly switchable fluorescent proteins (RSFPs), namely rsEGFP and rsEGFP2, which enables RESOLFT (REversible Saturable OpticaL Fluorescence Transition) -type super-resolution microscopy of living cells at low levels of light. The unique properties of these proteins overcome the limiting factors which were common to all RSFPs known so far and had therefore impeded their utilization for the RESOLFT approach. The rsEGFP variants exhibit fast switching speeds, low residual fluorescence in the off-state, and most notably, low switching fatigue which allows these proteins to be switched more than a thousand times. The established RSFP-based RESOLFT microscopy approach enables repeated imaging of cytoskeleton structures of living bacteria and mammalian cells as well as the observation of dendrites within an organotypic hippocampal slice of mice at resolution of better than $40 \mathrm{~nm}$. Imaging rates at up to $2 \mathrm{~Hz}$ reveal fast movements of peroxisomal vesicles and the ER (Endoplasmic Reticulum) in PtK2 cells by applying about one million times lower light intensities as compared to STED microscopy.

Further, the enhanced switching characteristics of rsEGFP facilitate its applicability as a medium for rewritable data storage. By adapting the superresolution microscopy principle to an all-optical data storage application, a method is presented which allows to write and read data spots in closer proximity than possible with conventional focusing. 


\section{Introduction}

The understanding of life requires the observation of processes on the level of its smallest subunit, the cell. The invention of the light microscope at the end of the $16^{\text {th }}$ century opened up the door to this micro world and subsequently allowed insight into the cellular processes. Starting with the discovery of the cell (Hooke, R. 1665), the scientific field of cell biology was born, followed by the detection of cellular substructures like the nucleus described by Antoni van Leeuwenhoek and later by Robert Brown in 1831.

Continious optimization in lens production techniques finally led to microscopy setups whose resolution seemed to be only limited by the diffraction barrier of light (Abbe, E. 1873)(see chapter II.1.c) to provide lateral resolution of $\sim 200 \mathrm{~nm}$ and axial resolution of $\sim 500 \mathrm{~nm}$. To achieve higher resolutions microscope approaches that did not rely on visible light were established like for example Transmission Electron Microscopy (TEM)(Kirkland, A.I. and Hutchinsons, L. 2007), Scanning Tunneling Microscopy (STM)(Binning, G. and Rohrer, H. 1982), and Atomic Force Microscopy (AFM)(Binnig, G. et al. 1986). All these light independent techniques require specific and complex sample preparation procedures that exclude the possibility to image living samples. In contrast, light microscopy allows the imaging of living cells and tissue. Consequently light microscopic techniques play a vital role in the life sciences.

The use of classical bright field microscopy is limited by the contrast arising from the differences in light absorption, scattering and diffraction between two objects. Therefore a variety of other techniques were established to efficiently enhance the contrast. Typical examples for these are Dark Field Microscopy (Reynolds, F.W. and Hesbacher, E.N. 1950), Phase Contrast Microscopy (Zernike, F. 1935), or Differential Interference Contrast (DIC) Microscopy (Barer, R. 1952). They utilize the detection of scattered light or interference effects due to objects of different refractive indices to enhance the contrast.

An other way to enhance the contrast is to stain the samples by using dyes. These dyes accumulate in specific structures based on their physical and chemical properties. For example, in histology, a hematoxylin and eosin stain is a typical method to enhance the contrast between the different substructures of 
cells (Avwioro, G. 2011). Another example is the discovery of the Golgi apparatus by histological staining in 1898 using silver nitrate. However, such classical dyes need to be highly concentrated to provide a detectable contrast. Therefore, the visualization of structures or proteins on the molecular level is not possible with such dyes.

A major breakthrough was achieved by the invention of fluorescence microscopy accompanied with the development of new labeling techniques like antibody staining which allow the marking of specific molecules and structures. Instead of detecting the absorption of light, fluorescence microscopy detects fluorescent light emitted by the fluorophores when illuminated with light of shorter wavelengths. This increases the contrast enormously because it is only dependent on the number of the detectable photons emitted by the fluorophore and the background noise, which is normally close to 0 . The resulting contrast even allows single molecule detection (Moerner, W.E. 2002).

Today a lot of different fluorescent dyes are available which can be used to specifically label subcellular structures for fluorescence microscopy. They vary from small chemical molecules consisting of only a few atoms to relatively large quantum dots of several nanometers. Often used dye families are for example the xanthenes, cyanines, naphthalenes, coumarines, pyrenes, and acridine derivates. This work focuses on a protein family that is used as fluorescent fusion markers in biological in vivo imaging: Fluorescent proteins (FPs) (see Chapter II.2).

\section{Fluorescence Microscopy}

\section{a. Fluorescence}

Fluorescence microscopy is based on detection of the fluorescent light emitted by fluorophores. Fluorescence is a specific type of luminescence. A Fluorophore can be excited by light of defined wavelengths depending on its absorption and excitation spectrum (Figure II.1). Subsequently spontaneous emission of a photon can take place. This emitted fluorescent light is red shifted in comparison 
to the excitation light. The wavelengths of the emitted fluorescent light are described by the emission spectrum of the fluorophore (Figure II.1).

Alternatively, a deexcitation can also occur thermally instead of the emission of a photon. The probability of a fluorophore to emit a photon rather than a thermal deexcitation is described by the fluo-

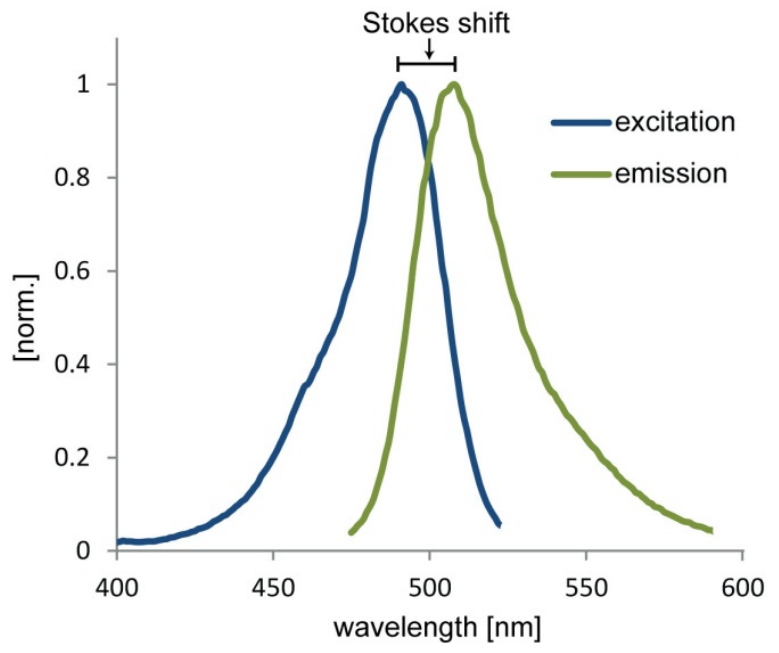

Figure II.1: Excitation (blue) and emission (green) spectrum of Alexa Fluor 488. rescence quantum yield $\Phi_{F l}$, which is the number of emitted photons per number of absorbed photons. The extinction coefficient $\varepsilon$ describes the probability of a fluorophore to absorb a photon.

The changes in the electronic excited states of a fluorophore during this process are described by the Jablonski diagram (Figure II.2). By the absorption of a photon an electron is excited from its ground state $\left(S_{0}\right)$ to one of the vibrational energy levels of the first exited state $\left(S_{1}\right)$. This is followed by a fast relaxation to the lowest vibrational state of $S_{1}$ by internal conversion. In the case that no thermal deexcitation takes place in a fluorophore in a specific time period (fluorescence lifetime), the electron relaxes back into one of the vibrational

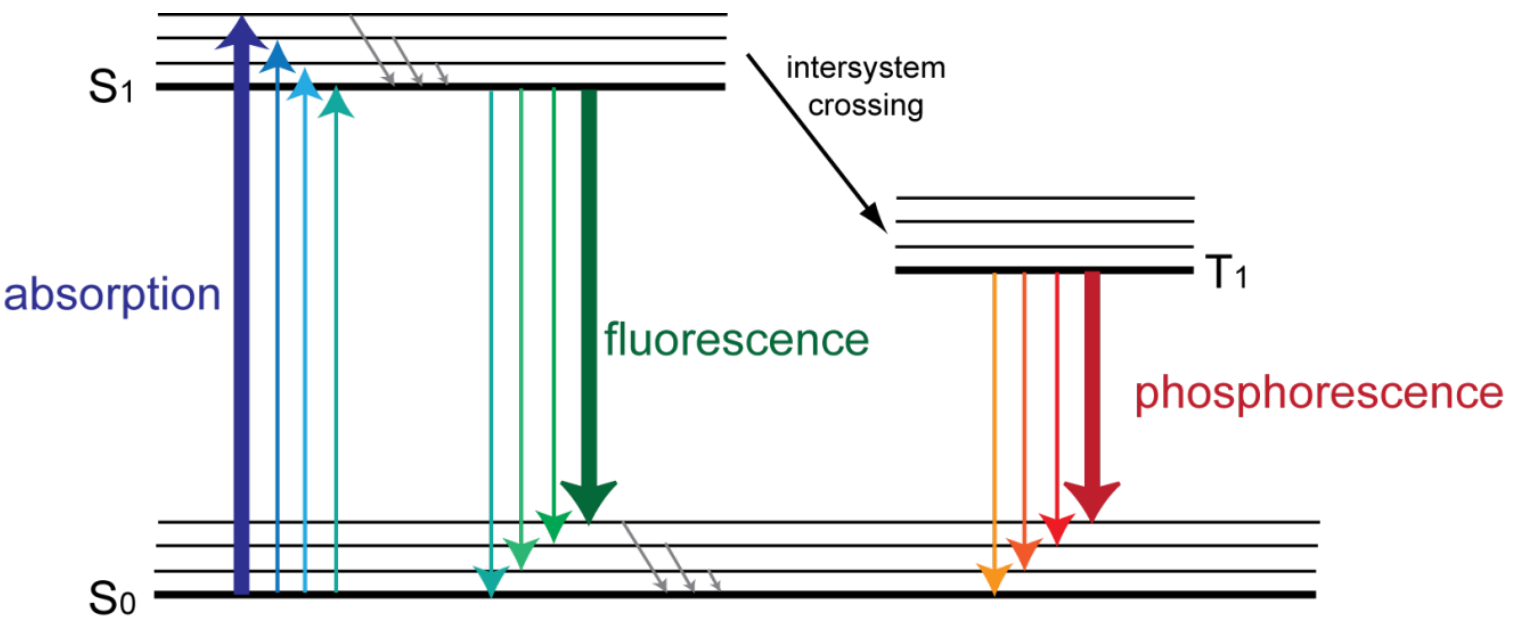

Figure II.2: The Jablonski diagram. 
energy levels of $S_{0}$ by emitting a photon (Jablonski, A. 1935; Jablonski, A. and Szymanowski, W. 1935).

The mentioned red shift of the fluorescence light in comparison to the excitation light is due to the loss of energy during relaxation to the lower vibrational states. This shift in wavelength is called "Stokes shift" (Figure II.1). The relaxation from and into different vibrational states is the main reason that a fluorophore can absorb and emit a wide spectrum of light instead of just absorbing light of a single wavelength and energy.

Besides relaxation from $S_{1}$ to $S_{0}$, an excited chromophore can convert to a metastable triplet state $T_{1}$ of lower energy by intersystem crossing, which is accompanied by a forbidden spin flip. The relaxation from $T_{1}$ to $S_{0}$ can take place either thermally or by phosphorescence (Figure II.2).

\section{Photobleaching}

Depending on their photostability, fluorophores can emit only a limited number of photons before they are photobleached. This photobleaching is a photo-induced, mostly irreversible chemical destruction of the fluorophore, resulting in a loss of the ability to fluoresce (Eggeling, C. et al. 1999; Pawley, J.B. 2006). It can occur from a variety of excited electronic states due to the fact that the higher energy level of an excited system is chemically more reactive than the ground state.

The different electronically excited states of a fluorophore exhibit different bleaching rates. Because the return from the $T_{1}$ or other dark states to the ground state is usually much slower than the $S_{1}-S_{0}$ decay, photobleaching from the $T_{1}$ and other dark states is much more effective and hence is a main parameter which determines the photostability of a fluorophore at low excitation intensities $\left(\leq \mathrm{W} / \mathrm{cm}^{2}\right)$.

At larger excitation intensities $\left(\geq \mathrm{kW} / \mathrm{cm}^{2}\right)$, the probability of the population of higher excited singlet and triplet (or dark) states $\left(S_{n}, T_{n}\right.$, and $\left.D_{n}\right)$ increases (Eggeling, C. et al. 1998; Dittrich, P.S. and Schwille, P. 2001). Especially in polar solvents such as water, these states couple quite efficiently with ionic states and result in very efficient photobleaching (Eggeling, C. et al. 1999). These photobleaching reactions require the absorption of an additional excitation photon 
in the $\mathrm{S1}, \mathrm{T} 1$ or D states, which very much depends on the fluorophore-specific excited state absorption cross sections. Due to its pronounced lifetime, the triplet (or dark) state is once again much more accessible to such non-linear, multi-step photobleaching at very large excitation intensities, often resulting in the fact that the fluorophore is immediately photobleached once it crosses to the long-living dark state system (Donnert, G. et al. 2007). All in all, there is a strong influence of the triplet state on the bleaching characteristics of a fluorophore.

Beside the excitation light intensities, the photobleaching characteristics of a fluorophore are strongly influenced by its environment such as $\mathrm{pH}$ and the presence of photo-reaction partners. Typical reaction partners are quenchers, proton donors, and dissolved atmospheric oxygen (Song, L. et al. 1995; Cordes, T. et al. 2011). The latter plays a very important role in photobleaching. On one hand it can reduce the triplet lifetime of a fluorophore, which may reduce the photobleaching reactivity but, on the other hand, very reactive oxygen may be formed upon this quenching process, leading to increased photobleaching activities. Therefore, the most efficient anti-fading solvents nowadays include triplet and dark state quenchers as well as radical scavengers (Vogelsang, J. et al. 2008).

The non-linear influence of the light intensity on photobleaching as well as the strong dependence on the environmental conditions complicates the determination of the photostability of a fluorophore. Therefore a comparison of the bleaching characteristics of two different fluorophores is only possible under the very same experimental conditions.

Hence, photostability is one of the most crucial properties of a fluorophore determining its usability for an application in fluorescence microscopy. It limits the maximal amount of detectable photons emitted by a single fluorophore and thereby heavily affects its brightness and subsequently the image quality.

\section{b. Fluorescence Microscopy Techniques}

The basic principle of fluorescence microscopy is to separate the fluorescent light of a fluorophore from the excitation light. Hence only the fluorescent light emitted from the labeled structures is detected. 
Classical epifluorescence microscopes use a white light source like mercury lamps for the excitation. A filter separates the excitation wavelength of light from all other wavelengths provided by the light source. The excitation light is reflected by a dichroic mirror into the objective lens, which focuses the light onto the sample to excite the fluorophores. The emitted fluorescence is partially collected by the same objective lens. The red shifted fluorescence light is separated from the excitation light by passing the dichroic mirror and the emission filter. Finally the fluorescence is detected either by using an ocular or a camera (Figure II.3.A).

In the wide field detection approach the whole area of interest is illuminated at once. A drawback of this approach is that the excitation light can also be absorbed by fluorophores above and below the focal plane. The fluorescent stray light leads to strong background signals, which can mask the signal of interest in the focal plane. This problem was strongly reduced by excluding the out-of-focus light with the invention of confocal laser scanning microscopy (CLSM).

A

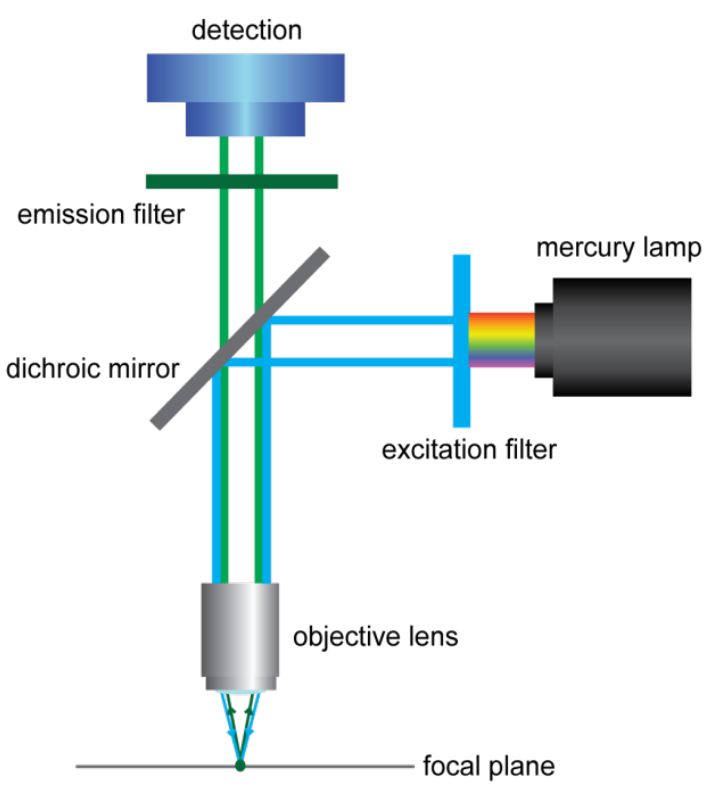

B

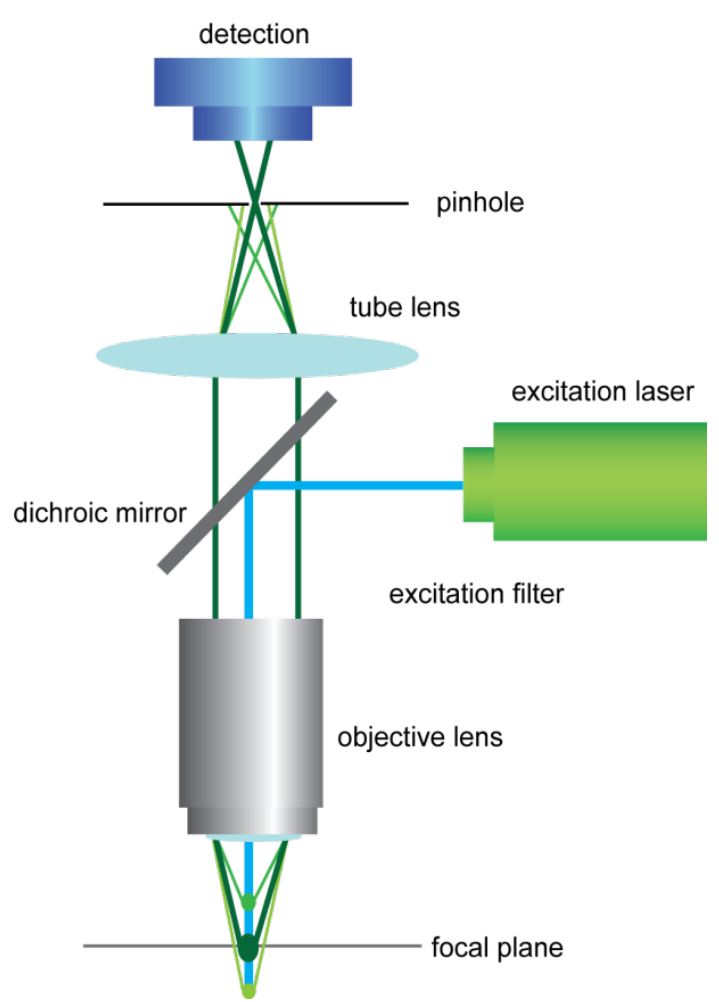

Figure II.3: Scheme of a classical epifluorescence microscope setup (A) and a CLSM setup (B). The pinhole prevents detection of fluorescence signals above and below the focal plane. 
The CLSM technique uses a laser as the excitation light source (Figure II.3.B). The coherent light is focused by the objective lens to a small diffraction limited spot. The fluorescence signal emitted from this spot is detected by a photomultiplier tube (PMT) or an avalanche photodiode (APD). At the image plane a confocal pinhole is inserted into the detection path. This pinhole blocks light from above and below the focal plane, resulting in an enhancement of resolution along the z-plane. By scanning the beam over the sample, the image can be constructed based on the fluorescence intensity value of each scanning position.

\section{c. The Diffraction Barrier and Super-Resolution Microscopy}

Being a non-invasive imaging technique, light microscopy is indispensable for the life sciences. However, a major drawback of using light is its focusing limitation dependent on the wave nature of light, which has restricted the achievable resolution in light microscopy for decades.

The resolution of an imaging system is defined by the smallest distance at which two objects can still be discerned. Ernst Abbe described for the first time the limitation in optical resolution based on the restriction of focusing light to a spot below a defined size (Abbe, E. 1873; Abbe, E. 1880). The lateral resolution limit $(d)$ is given by the wavelength of light $(\lambda)$, the refractive index $(n)$, and the semi aperture angle $(\alpha)$ of the objective lens. The product of the refractive index and the sine of the semi aperture angle is defined as the numerical aperture (NA) of an objective lens:

$$
d=\frac{\lambda}{2 n \sin \alpha}=\frac{\lambda}{2 N A}
$$

The axial resolution is given by:

$$
d=\frac{\lambda}{2 n \sin ^{2} \alpha}
$$

This means that objects at a separation distance below $\sim 200 \mathrm{~nm}$ in lateral and $500 \mathrm{~nm}$ in axial direction can not be discerned from each other by classical light 
microscopy. This changed with the invention of super-resolution microscopy techniques.

The invention of the STED (Stimulated Emission Depletion) microscopy by Stefan W. Hell allowed for the first time to overcome the diffraction barrier of light without any theoretical limitation (Hell, S.W. and Wichmann, J. 1994; Hell, S.W. 2007). In the most common way, STED microscopy is realized in a CLSM setup by adding a second beam of light with longer wavelength (Figure II.4.A). This doughnut shaped beam is overlaid with the excitation beam to prevent the excited molecules from fluorescing in the periphery of the excitation spot. STED describes a photo physical process in which an excited fluorophor can be forced back to the ground state when interacting with a photon of nearly the same energy. This is accompanied with the emission of a copy photon with exactly the same wavelength as the STED light. By preventing the fluorophores in the periphery of the excitation spot to fluoresce, only those fluorophores in the very center of the spot, where the STED

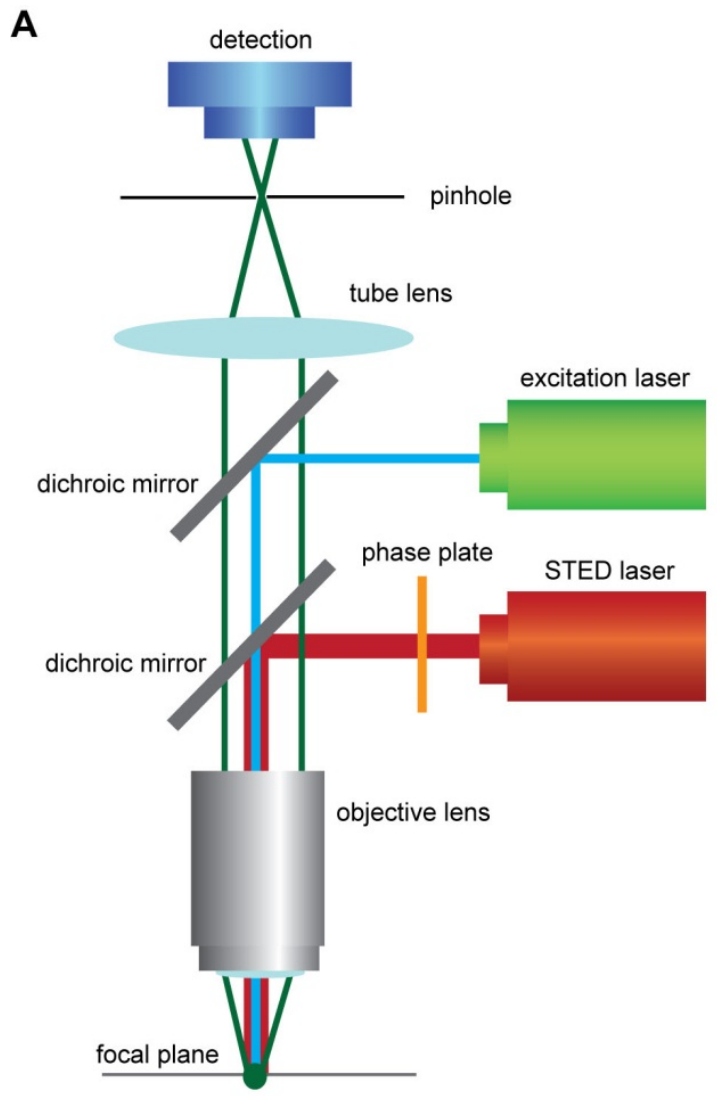

B

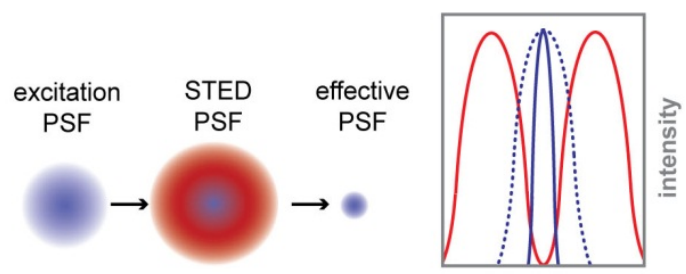

Figure II.4: The STED microscope. (A) Scheme of a STED microscope setup. A phase plate transforms the beam into a doughnut shape. The beam is overlaid with the excitation beam. (B) Overlaying the beams leads to an effectively smaller detection PSF. The graph shows intensity profiles of the excitation (blue dotted), STED (red), and effective PSF (blue solid line).

intensity is zero, can still emit fluorescence light. This leads to an effectively smaller point spread function (PSF) describing the detection profile (Figure II.4.B). Pushing the STED process more and more into saturation by increasing the intensity of the STED beam, the effective excitation PSF becomes smaller and smaller. Its size is no longer limited. The resolution is now determined by the 
following equation, with $I$ being the intensity of the STED beam and $I_{s}$ being the characteristic STED intensity required for saturating the transition:

$$
d \approx \frac{\lambda}{2 N A \sqrt{1+\frac{I}{I_{S}}}}
$$

An intrinsic drawback of STED microscopy is the requirement of high light intensities in the $\mathrm{MW} / \mathrm{cm}^{2}$ to $\mathrm{GW} / \mathrm{cm}^{2}$ range to effectively saturate the stimulated emission processes. This may induce phototoxic effects in living samples.

However, the basic idea to increase the resolution by switching of fluorophores between fluorescent and non-fluorescent states is not only limited to STED. In principle, each process of switching between two different and discernable fluorescent states can be used to increase resolution. This is why all targeted switching based super-resolution techniques including STED microscopy are summed up into one class: Reversible Saturable Optical Fluorescence Transition (RESOLFT) microscopy (Hell, S.W. 2003; Hell, S.W. et al. 2003; Hofmann, M. et al. 2005).

In 2006 a second subclass of super-resolution microscopy approaches was established (Betzig, E. et al. 2006; Rust, M.J. et al. 2006). Instead of targeted switching, the fluorophores are switched randomly in time while detecting the whole area of interest using a wide field approach and a fast CCD camera for detection. The number of molecules in the detectable on state is kept so low that just single molecule events appear. Based on the knowledge that each fluorescence signal is emitted from a single molecule, the molecule can be localized by calculating the center of mass (Heisenberg, W. 1930). Afterwards, the detected molecules are switched off and a new subset of fluorophores is detected. The final image is a localization map consisting of all sequentially detected single molecule events (Figure II.5). As in RESOLFT microscopy, different types of switching can be used for these single molecule switching (SMS) approaches. For example, photoactivation localization microscopy (PALM) uses photoactivatable fluorescent proteins (Betzig, E. et al. 2006), stochastic optical reconstruction microscopy (STORM) is based on photoswitchable 
chemical dyes (Rust, M.J. et al. 2006), ground state depletion microscopy (GSDIM) pumps fluorophores in the triplet state and detects their recovery (Fölling, J. et al. 2008), and PALM with independently running acquisition (PALMIRA) utilizes reversibly switchable fluorescent proteins (Egner, A. et al. 2007).

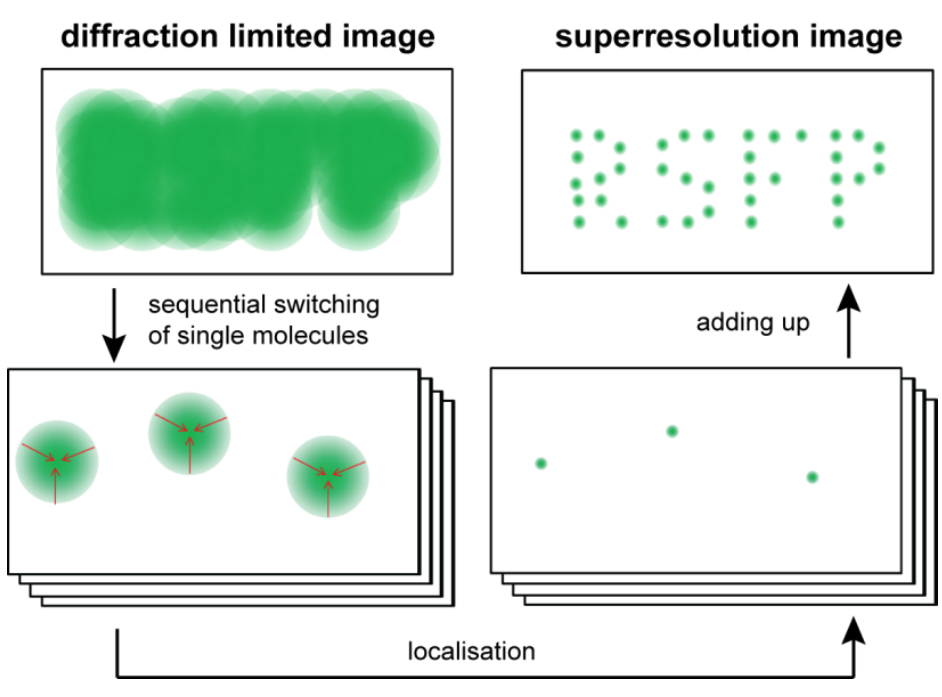

Figure II.5: The principle of SMS microscopy. By sequentially switching and localizing single molecules a super-resolution image can be reconstructed.

RESOLFT as well as SMS microscopy has the theoretical potential to reach molecular resolution. However, in practice, the methods are mostly limited by the properties of the available dyes. The requirements concerning the dyes are different for both super-resolution methods.

In case of SMS microscopy the fluorophores just need to undergo only one single switch. The localization precision depends on the number of counted photons per single molecule event. Hence the most important requirement for dyes used in SMS microscopy is high brightness.

RESOLFT microscopy allows the detection of several fluorophores at once. Hence brightness is not such a strong limitation as it is in SMS microscopy. However, to achieve an $m$-fold improvement in resolution along one dimension requires $\sim m$ switching cycles, whereas a resolution improvement in all three dimensions requires $\sim m^{3}$ cycles. For example, an improvement of a factor of ten in 3D requires $\sim 1000$ cycles. Consequently, the most limiting factor for RESOLFT microscopy is the number of switching cycles a fluorophore can undergo before it is bleached.

The strong influence of the dyes on the imaging performance makes the generation and improvement of fluorophores a major challenge in enhancing super-resolution microscopy approaches. This work therefore focuses on the generation and improvement of fluorescent proteins to enable and overcome the diffraction barrier of light by a RESOLFT technique. 


\section{Fluorescent Proteins}

The discovery of fluorescent proteins (FPs) led to a renaissance in fluorescence microscopy. The possibility to use them as genetically encoded fluorescence markers revolutionized live cell analyses. For the first time, proteins of interest could be visualized without treating cells by fixation and staining with chemical dyes. The Nobel Prize for chemistry 2008 awarded to Osamu Shimomura, Martin Chalfie, and Roger Y. Tsien for discovery and development of the green fluorescent protein illustrates the importance of FPs for the life sciences.

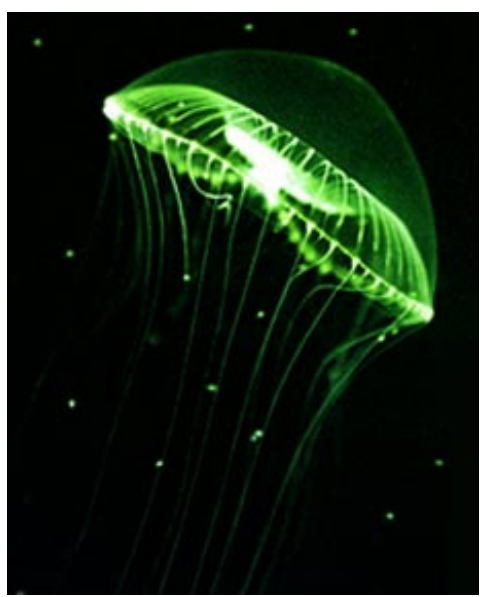

Figure II.6: The jellyfish Aequoria Victoria (source: http://www.univie.ac.at).

Osamu Shimomura isolated the first FP known from the bioluminescent jellyfish Aequoria victoria (Shimomura, O. et al. 1961)(Figure II.6). It absorbs blue light and fluoresces green, consequently it was called "Green Fluorescent Protein" (Aequoria victoria GFP = avGFP). Its natural function in the jellyfish is to work as a FRET (Förster Resonance Energy Transfer) acceptor. It expands the luminescence spectrum of aequorin to longer wavelengths (Morise, $\mathrm{H}$. et al. 1974). Finally it took 31 years from isolating avGFP to the determination of its cDNA Sequence (Prasher, D.C. et al. 1992). avGFP took off on its triumphal course in 1994 when it was shown that this protein can be expressed in eukaryotic as well as in prokaryotic organisms while keeping its fluorescent functionality. Furthermore, it can be fused $\mathrm{N}$ - or C-terminally to other proteins by cloning (Chalfie, M. et al. 1994; Inouye, S. and Tsuji, F.I. 1994; Wang, S. and Hazelrigg, T. 1994). Expressing the fusion protein results in a functional and fluorescently marked protein for fluorescence microscopic live cell studies. This is facilitated by the fact that the avGFP chromophore is formed completely autocatalytically out of three defined amino acids. This maturation reaction needs no co-factors except molecular oxygen (Heim, R. et al. 1994).

avGFP was the basis of the first enhanced and optimized FP variants finally leading to the well known "Enhanced Green Fluorescent Protein" (EGFP) (Clontech, CA, USA / GenBank Accession \#U55762) (Thastrup, O. et al. 1995). 
EGFP is based on GFPmut1 (Cormack, B.P. et al. 1996), which contains the mutations F64L and S65T (Heim, R. et al. 1995). Further, the DNA sequence coding for EGFP was adapted to human codon-usage preferences (Haas, J. et al. 1996). In contrast to avGFP, which exhibits a major absorption peak at $\sim 395$ $\mathrm{nm}$ (protonated form of the chromophore) and a minor one at $\sim 475 \mathrm{~nm}$ (deprotonated form of the chromophore), the chromophore of EGFP is completely deprotonated, which results in a single absorption peak at $488 \mathrm{~nm}$ (see also Chapter II.b). In comparison to avGFP, the brightness of EGFP is increased 35-fold when they are excited with light of $488 \mathrm{~nm}$ (Cormack, B.P. et al. 1996). These optimized fluorescence characteristics make EGFP one of the most favorable FPs up to now.

In 1999 it turned out that a multiplicity of avGFP homologous FPs can be found in non-luminescent Anthozoa (Matz, M.V. et al. 1999). This extended the fluorescence color spectrum of available FPs to the red range. Up to now further FPs could be found in several classes of Cnidaria (Labas, Y.A. et al. 2002; Matz, M.V. et al. 2006; Alieva, N.O. et al. 2008; Aglyamova, G.V. et al. 2011), but also in Arthropoda (Shagin, D.A. et al. 2004), in the Chordata Amphioxus (Deheyn, D.D. et al. 2007) and in Copepoda (Hunt, M.E. et al. 2010). DsRed played a central role in the newly discovered FPs (Fradkov, A.F. et al. 2000; Yarbrough, D. et al. 2001), the first identified red fluorescent protein. It was purified from the coral Discosoma sp.. Most of the wild type FPs have the tendency to oligomerize. This is a major drawback since the tendency of the FPs to interact can influence the localization pattern of the fusion protein. The tetrameric characteristic of DsRed made further mutagenesis necessary, leading to the generation of the optimized monomeric mRFP1 (monomeric Red Fluorescent Protein)(Campbell, R.E. et al. 2002). Further developments resulted in new FP variants covering the whole yellow and red parts of the light spectrum (Shaner, N.C. et al. 2004).

\section{a. The Overall Structure of Fluorescent Proteins}

In 1996 the structure of avGFP was solved by X-ray crystallography (Ormo, M. et al. 1996), followed by the structure determination of a multiplicity of other FPs. They consist of a single polypeptide of $\sim 220$ amino acids resulting in a molecular 
weight of $\sim 26 \mathrm{kDa}$. The overall structure of FPs is strongly conserved, but the amino acid sequence similarity can be less then $25 \%$ (Evdokimov, A.G. et al. 2006). They all form an 11 stranded $\beta$-barrel with a length of $\sim 42 \AA$ and a diameter of $\sim 24 \AA$ (Figure II.7). Through the center of the barrel runs an $\alpha$-helix of $\sim 14$ amino acids. In the very center of this helix the chromophore is built autocatalytically out of three amino acids. It is stabilized inside the barrel by a complex hydrogen bonding network. The barrel shields the chromophore from external influences and plays an important role in catalyzing the chromophore maturation. Hence the barrel as well as the chromophore itself, influences the fluorescence properties of an FP.
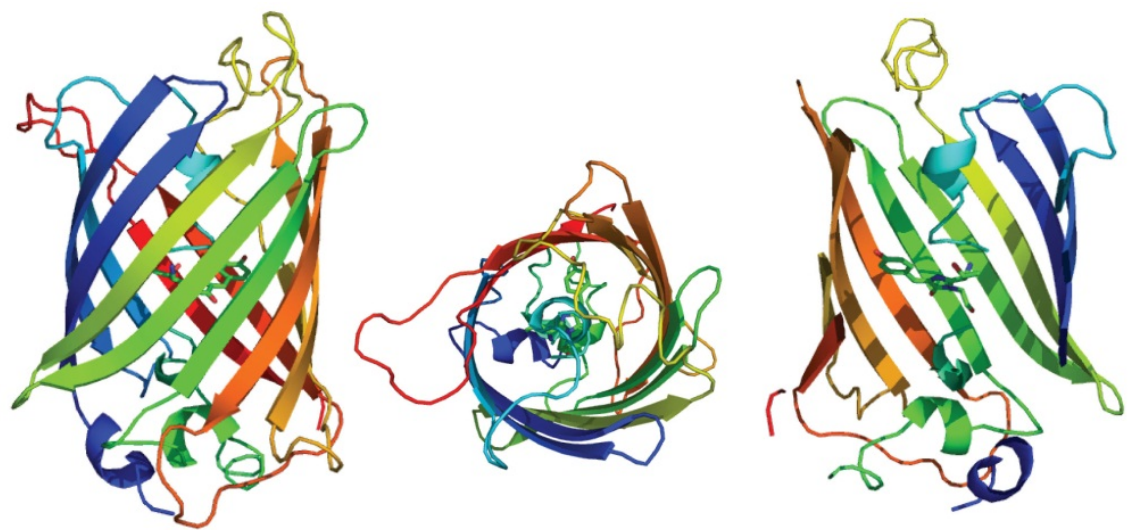

Figure II.7: The protein structure of fluorescent proteins. X-ray structure of avGFP (Ormo, M et al. 1996)(PDB-ID: EMA1) from side (left), top (middle) and inside view (right), illustrated with the "cartoon" function and colored with the "Chainbow" option of PyMol. The color orientation is from blue ( $\mathrm{N}$-terminus) to red (C-terminus). The chromophore is illustrated in "lines" mode (C atoms green, $\mathrm{O}$ atoms red and $\mathrm{N}$ atoms blue).

\section{b. The Chromophore and Its Environment - Maturation,}

\section{Conformation, Protonation}

The chromophore maturation is catalyzed by the FPs itself. No co-factors except molecular oxygen are needed. This allows a functional FP formation in a variety of different cell types and organelles, making FPs widely applicable in life sciences. The $\pi$-electron-system of GFP like chromophores consists of a $p$ hydroxyphenyl and an imidazole ring connected by a methine bridge. It is formed out of three amino acids which, in case of avGFP, are Ser65-Tyr66-Gly67 (Figure II.8.A). While Gly67 is mandatory for chromophore maturation (Barondeau, D.P. et al. 2003), the first and second amino acids are variable. However, the $\operatorname{Tyr}_{66}$ is 
conserved in all known wild type FPs. The maturation time of the chromophore is determined by the chromophore surrounding amino acids (Bevis, B.J. and Glick, B.S. 2002) and usually lies in a time range of a few hours.

\section{Maturation}

The maturation reactions to form the chromophore are still a matter of discussion in their details (Craggs, T.D. 2009; Strack, R.L. et al. 2010; Wachter, R.M. et al. 2010). In the following the most prominent reaction model is presented.

The chromophore maturation reaction requires a complete folding of the protein (Reid, B.G. and Flynn, G.C. 1997). An untypical tight-turn conformation of the ahelix may bring the Gly67 amide nitrogen (N67) and the carbonyl carbon of Ser65 (C65) into van der Waals contact providing an optimal orbital orientation for a nucleophilic attack of N67 (Barondeau, D.P. et al. 2005; Craggs, T.D. 2009). This results in the formation of a 5-membered ring (Niwa, H. et al. 1996)(Figure II.8.B). The side-chain residue of Gly67 is small enough to sterically allow this cyclization reaction, which explains its essential function in chromophore formation. It is discussed that the cyclization reaction is facilitated by Arg96 acting as an electrostatic catalyst (Figure II.8.C). The second step in maturation is an enolization reaction. The Ca of Tyr66 is deprotonated which may be promoted by Glu222 (Barondeau, D.P. et al. 2003; Sniegowski, J.A. et al. 2005; Wachter, R.M. et al. 2010). It is suggested that the 5-ring of the enolat form can be in a hydrated or a dehydrated state. The next step is the formation of an imine intermediate by a 2 electron oxidation with molecular oxygen. In line with this reaction, hydrogen peroxide is produced (Zhang, L. et al. 2006). The final reaction in chromophore maturation is a dehydration reaction by deprotonating the $C \beta$ of Tyr66, which results in the formation of the methine bridge (Rosenow, M.A. et al. 2005; Pouwels, L.J. et al. 2008) It is suggested that this dehydration reaction includes a radical intermediate (Barondeau, D.P. et al. 2007). Finally, both ring systems are connected to one delocalized m-electron system which absorbs blue light.

The red shifted absorption and fluorescence of the DsRed chromophore is based on an extension of the rm-electron system into the first of the three chromophore amino acids Gln66, Tyr67, and Gly68 (Figure II.8.A). For a long time it was suggested that a GFP like chromophore structure is an intermediate 
in the red chromophore type formation (Gross, L.A. et al. 2000; Jakobs, S. et al. 2000). Today it is widely accepted that there is a branching point between green and red chromophore formation after the formation of the imine intermediate (Verkhusha, V.V. et al. 2004; Strack, R.L. et al. 2010). An air oxidation, which is suggested to be triggered by a proton abstraction at the $\mathrm{C} \alpha$ of $\mathrm{Gln} 66$, produces an acylimine and sets the course for red chromophore formation (Figure II.8.B). This seems to be mediated by Glu215 (Figure II.8.C). A hydroxylation followed by

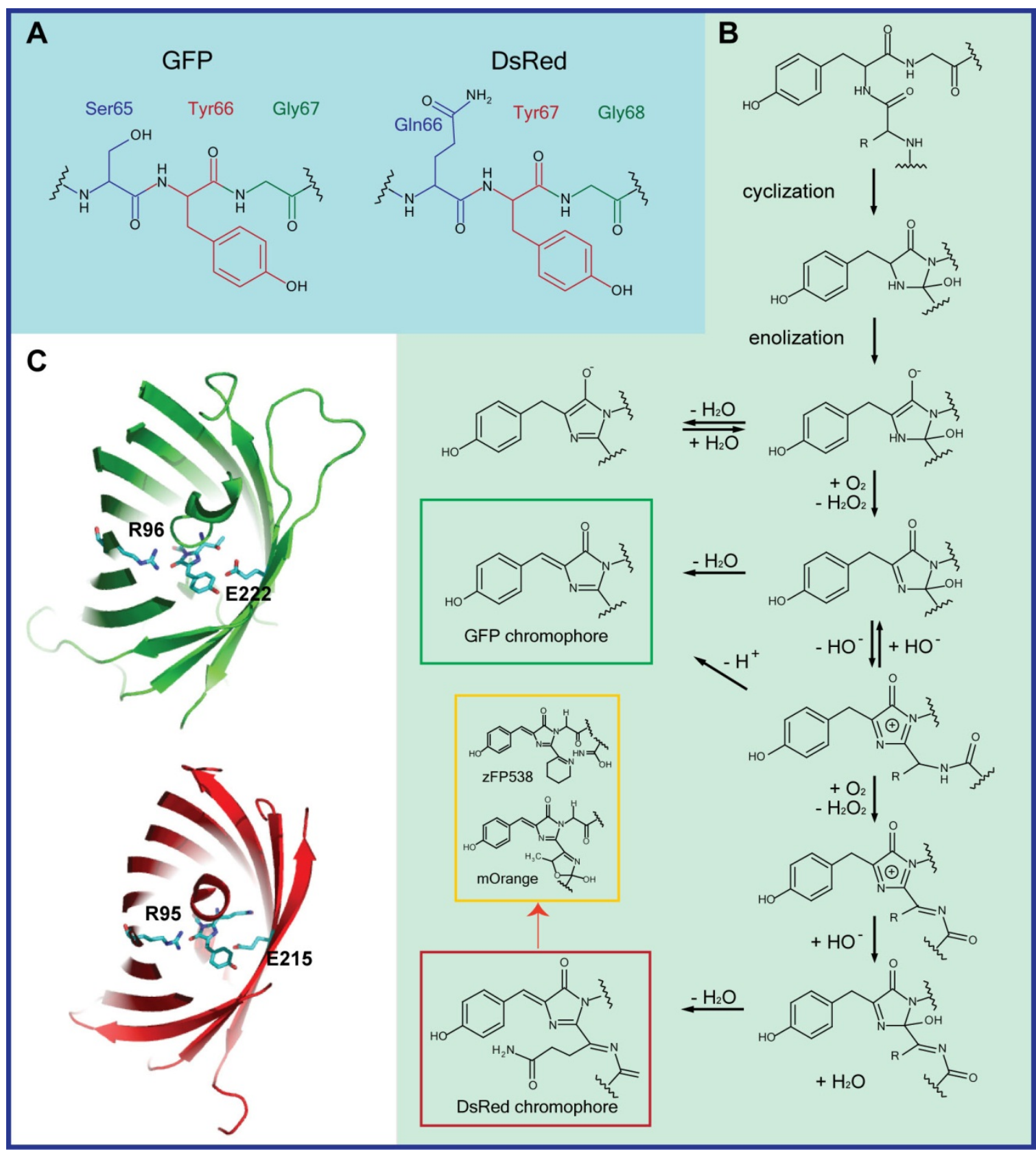

Figure II.8: Maturation model of GFP and DsRed type chromophores. (A) Primary structure of the three amino acids building the chromophore in GFP (left) and DsRed (right). (B) Reaction cascade of GFP- and DsRed-like chromophores maturation. $R$ is a variable representing the residue of the first GFP- or DsRed-type chromophoric amino acid. (C) Structures of GFP (top) and DsRed (bottom) highlighting amino acids influencing the chromophore formation. 
a water subtraction leads to the final DsRed chromophore, which absorbs in the green and emits in the red light range (Tubbs, J.L. et al. 2005; Strongin, D.E. et al. 2007).

It is known from other FPs with a DsRed chromophore that additional maturation reactions can take place. The reason for this is the reactivity of the acylimine group acting as an electron sink (Pakhomov, A.A. and Martynov, V.I. 2009; Wachter, R.M. et al. 2010). Examples for this modification are the chromophores of mOrange and zFP538. In mOrange a cyclization takes place between the hydroxyl group of Thr66 and the carbonyl carbon of the acylimine (Shu, X. et al. 2006). In zFP538 it is suggested that the amino acid residue of Lys66 cyclizes with its own Ca atom accompanied with a chain break of the polypeptide. In both cases the modification results in a shortened m-electron system. These proteins fluoresce in the yellow part of the visible light spectrum.

\section{Conformation}

The conformation a chromophore adopts is determined by its surrounding amino acids. Nearly all known FPs exhibit a coplanar chromophore in the cis conformation. Known exceptions are eqFP611 isolated from the sea anemone Entacmaea quadricolor (Petersen, J. et al. 2003) and TagRFP, a monomeric and optimized variant of eqFP578 from the same species (Merzlyak, E.M. et al. 2007; Malashkevich, V.N. et al. 2010). Both brightly fluorescent proteins show a coplanar chromophore in the trans conformation. Other known proteins with a non-coplanar trans chromophore like Rtms 5 derived from Montipora efflorescens do not fluoresce (Prescott, M. et al. 2003). These examples illustrate the strong influence of the chromophore conformation and planarity on the probability to fluoresce. Especially a loss in coplanarity seems to decrease the quantum yield (Schenk, A. et al. 2004; Yan, W. et al. 2009; Brakemann, T. et al. 2010).

A further important factor influencing quantum efficiency is the stabilization of the chromophore conformation. An avGFP chromophore in solution exhibits nearly no fluorescence (Niwa, H. et al. 1996). The stabilization of the chromophore reduces the probability of non radiative dissipation of energy by movements. This stabilization in FPs between the chromophore and surrounding amino acids is facilitated by a hydrogen bonding network. 


\section{Protonation}

The mature chromophore can adopt a neutral and an anionic form due to the protonation state of the apical oxygen of the chromophore, which is influenced by the water network with surrounding amino acids. The change in charge by protonation or deprotonation of a chromophore affects its photo-physical properties by influencing its $\pi$-electron system. Thus, the absorption spectrum of an anionic chromophore is red-shifted by $\sim 80 \mathrm{~nm}$ compared to the one of the neutral form.

avGFP is an example for a chromophore adapting both forms in an equilibrium. This is illustrated by the absorption spectrum of avGFP showing two peaks, a major one at $\sim 395 \mathrm{~nm}$ and a smaller one at $\sim 475 \mathrm{~nm}$, representing the protonated and deprotonated chromophor states, respectively (Heim, R. et al. 1994). The two different forms are stabilized by two alternative water networks (Figure II.9), which appear to be dependent on the conformation of the amino acid residue of Thr203. Surprisingly, an excitation into the protonated as well as
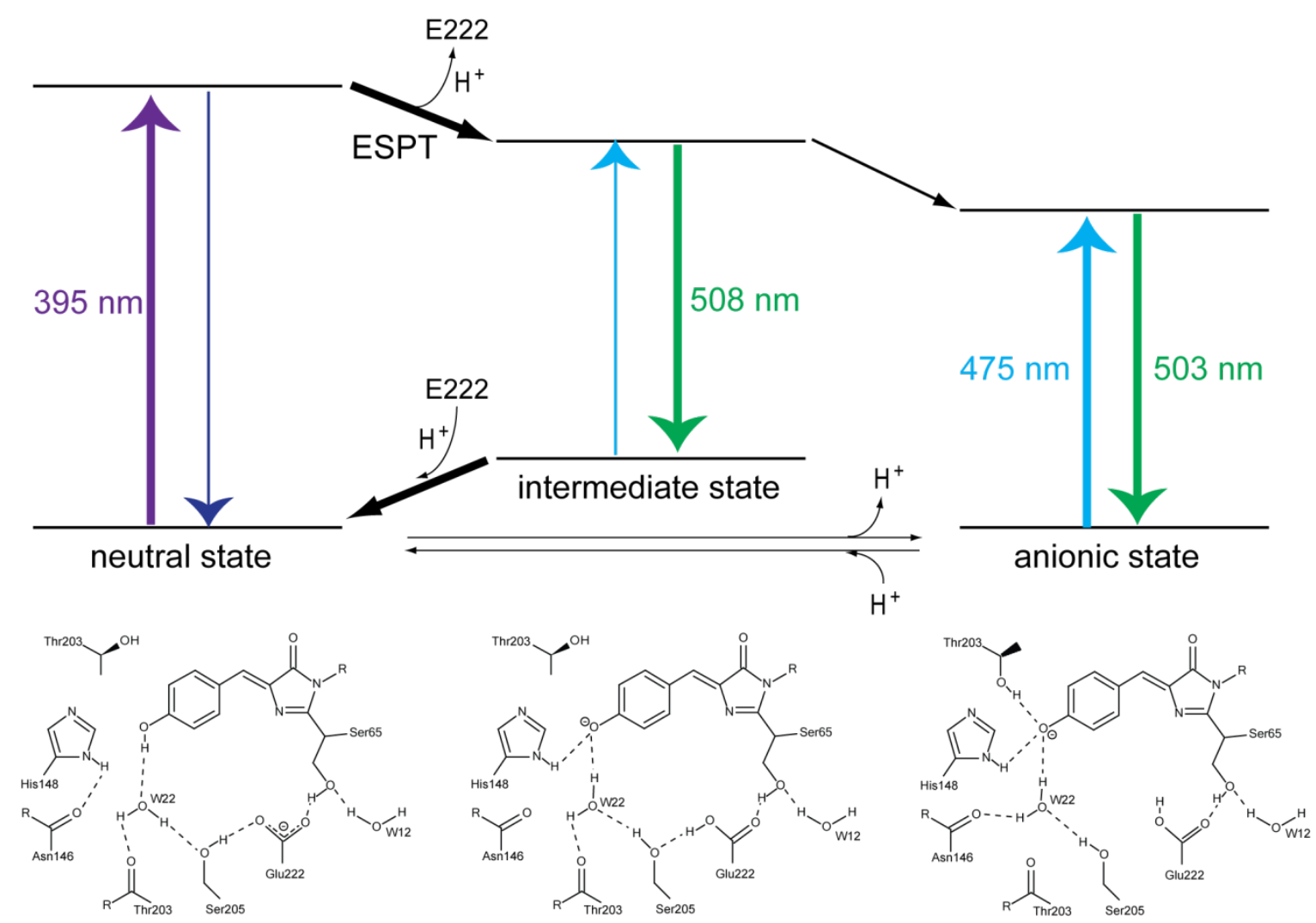

Figure II.9: Protonation states and ESPT in avGFP. Excitation of the neutral state at $395 \mathrm{~nm}$ leads to a conversion to the intermediate state by ESPT transferring a proton from the apical hydroxyl group of the chromophore to Glu222 by a rearrangement of the hydrogen network. 
into the deprotonated absorption bands yields green fluorescence at $\sim 508 \mathrm{~nm}$ (395 nm excitation) and $503 \mathrm{~nm}$ (475 nm excitation). The reason for this is a process called Excited State Proton Transfer (ESPT).

When the neutral chromophore is excited, a proton transfer from the chromophoric tyrosyl to Glu222 may occur (Figure II.8). The resulting excited intermediate form of the chromophore fluoresces at $\sim 508 \mathrm{~nm}$ (Brejc, K. et al. 1997; Palm, G.J. et al. 1997). ESPT occurs in the picosecond time range, which is much faster than the fluorescence lifetime of the neutral chromophore form. This makes the ESPT process very efficient and it is the reason why nearly no fluorescence at $\sim 450 \mathrm{~nm}$ appears from the excited neutral state (Lill, M.A. and Helms, V. 2002; van Thor, J.J. et al. 2005; van Thor, J.J. et al. 2005).

The intermediate chromophore form is not stabilized by Thr203. Subsequently the intermediate ground state converts back into the neutral form (Wiehler, J. et al. 2003). A non-radiative transition from the excited intermediate to the excited anionic state is possible but rare, due to the fact that the process is slow and implements the conformational change of Thr203 (Figure II.9). This can be detected by an increase in the absorption band at $475 \mathrm{~nm}$ after excitation with UV light (Chattoraj, M. et al. 1996). This photoconversion is mainly reversible by a ground state relaxation from the deprotonated to the protonated state (Craggs, T.D. 2009). The irreversible fraction is due to a further transition from the neutral to the anionic state accompanied by a decarboxylation reaction of Glu222 (Craggs, T.D. 2009).

The hydrogen network of avGFP illustrates the strong influence of the chromophoric environment onto the chromophore and the fluorescence properties exemplarily. The understanding and tuning of the chromophore as well as its environment opened up the way to design new FP variants.

\section{c. Tuning the Spectral Properties of Fluorescence Proteins}

Beside the discovery of new FPs in a variety of species, the generation and optimization of new variants finally led to a color table of proteins spanning the whole visible light spectrum (Figure II.10). This multiplicity of different FPs has enabled multicolor fluorescence microscopy and allowed the detection of several 
differently marked proteins simultaneously. Since the fluorescence properties of FPs are influenced by the chromophoric system itself and by its surrounding amino acids, both factors can be changed to alter the fluorescence characteristics.

As already mentioned, the second chromophore amino acid in all wild type FPs is a tyrosine. However, tyrosine is not essential for chromophore

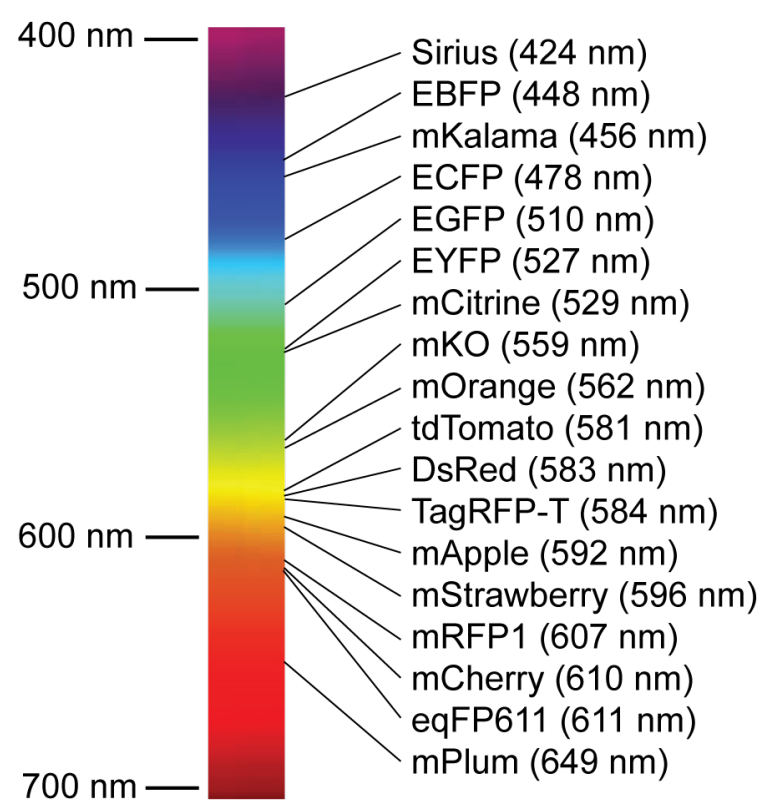
maturation. Exchanging it against other aromatic amino acids changes the $\pi-$

Figure II.10: The emission wavelengths of different fluorescent proteins.

electron system of the resulting chromophore and leads to different variants with a blue shifted fluorescence spectrum (Heim, R. et al. 1994; Wachter, R.M. et al. 1997). In the Blue Fluorescent Protein (BFP), the tyrosine is replaced by a histidine (Figure II.11.A), which emits at 448nm (Heim, R. et al. 1994). An exchange against tryptophane in the Cyan Fluorescent Protein (CFP) leads to an emission at 478nm (Figure II.11.B). The FP Sirius with a phenylalanine as the second chromophore amino acid exhibits the so far shortest emission wavelength at $424 \mathrm{~nm}$ (Tomosugi, W. et al. 2009). Other blue FP variants have a GFP type chromophore. In these cases

A

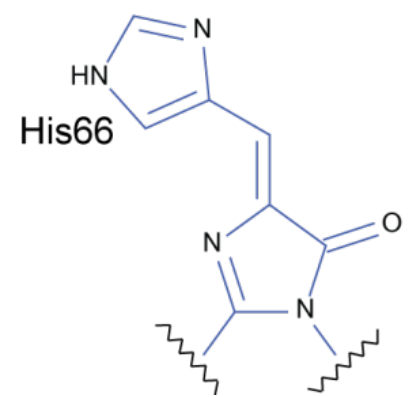

B

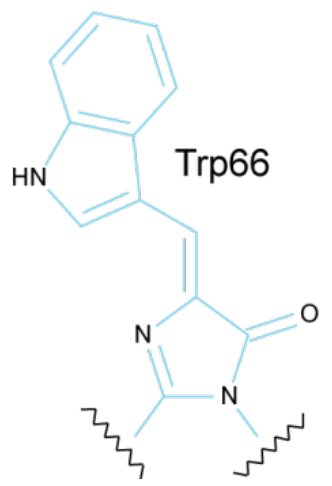

C

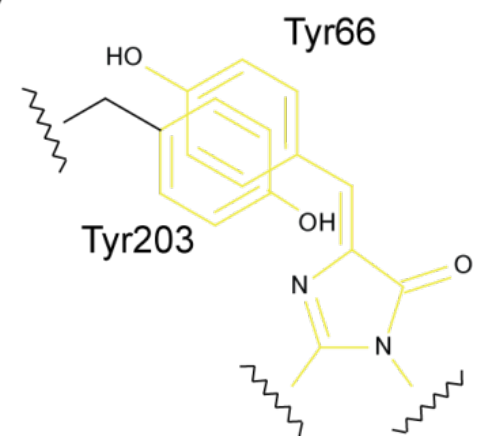

Figure II.11: The chromophore structure of BFP (A), CFP (B), and YFP (C). In comparison to a Tyr66 chromophore, His66 in BFP and Phe66 in CFP exhibiting shortened m-electron systems. A $\pi$ stacking interaction between the YFP chromophore and Tyr203 leads to an effective extension of the m-electron systems. 
changes in the chromophore environment cause the blue shift. In mKalama the GFP-type chromophore is blocked in the protonated state and ESPT is interrupted (see Chapter II.2.b)(Ai, H.W. et al. 2007), which results in blue fluorescence emission at $456 \mathrm{~nm}$. Another example of changes in the fluorescence spectrum induced by the chromophore environmental is amFP486, which was isolated from Anemonia majano. Here, the blue shift is due to a $\pi-$ stacking interaction between the hydroxyphenyl ring of the chromophore and the positive charged His199 (Henderson, J.N. and Remington, S.J. 2005).

A red shift in the emission spectra of a GFP type chromophore could be achieved in the Yellow Fluorescent Protein (YFP). A m-stacking interaction between the chromophore and Tyr203 results in a fluorescence emission at $528 \mathrm{~nm}$ (Figure II.11.C).

Orange and red variants of FPs are based on DsRed-type chromophores implying the elongation of the $\pi$-electron system by oxidation of the first chromophoric amino acid (see Chapter II.2.b). Mutagenesis of chromophore surrounding amino acids in mRFP1 (Campbell, R.E. et al. 2002) led to a collection of new FPs, called "mFruits", covering the whole spectral range from orange to red (Shaner, N.C. et al. 2004; Wang, L. et al. 2004). The most reddish FPs known so far are mPlum (Wang, L. et al. 2004), mNeptune (Lin, M.Z. et al. 2009), and TagRFP657 (Morozova, K.S. et al. 2010), which emit fluorescence at $649 \mathrm{~nm}, 650 \mathrm{~nm}$, and $657 \mathrm{~nm}$. Interestingly, mPlum still absorbs light at $~ 588 \mathrm{~nm}$. This unusual large Stokes shift is due to an increased flexibility of the chromophore environment (Abbyad, P. et al. 2007).

The fluorescene wavelengths of FPs are just one of a variety of fluorescence properties which can be influenced by changing the chromophore surrounding amino acids. The enhancement of a variety of crucial FP characteristics is described in the following. 


\section{d. Optimizing Fluorescent Protein Characteristics for Specific}

\section{Needs}

Depending on the designated application, an FP has to fulfill different requirements. Therefore a variety of different FPs was optimized focusing on different fluorescence characteristics.

\section{Brightness}

The brightness of a fluorophore, determined by its molecular extinction coefficient and its quantum yield, is a crucial characteristic directly influencing the contrast of a fluorescence microscope image. Hence a major focus in FP generation is enhancing or keeping the proteins brightness. The fluorescence brightness can be easily quantified. Consequently, brightness enhancement can be achieved by directed and random mutagenesis (Leung, D.W. et al. 1989; Sawano, A. and Miyawaki, A. 2000) in combination with high throughput screening systems like a Fluorescence Activated Cell Sorter (FACS). Examples for the optimization of an FP brightness are the avGFP mutant GFPmut1 (Cormack, B.P. et al. 1996), mCherry (Shaner, N.C. et al. 2004), which is generated on basis of mRFP1, and Citrine, a bright variant of YFP (Heikal, A.A. et al. 2000; Griesbeck, O. et al. 2001).

\section{pH Stability}

Changes in $\mathrm{pH}$ can influence the protonation state of the chromophore and thus result in changes of the fluorescence properties. Depending on its $\mathrm{pH}$ stability, an initially deprotonated fluorescent chromophore gets protonated under more acidic conditions. This is determined by its $\mathrm{pKa}$ value. This value reflects the $\mathrm{pH}$ at which the fluorescence equals $50 \%$ of the maximal brightness (Chudakov, D.M. et al. 2010). Hence low pH stability limits the usability of the FP because different cell types and organelles exhibit different $\mathrm{pH}$. An example for an FP with increased $\mathrm{pH}$ stability is Citrine, which was generated on basis of YFP (Heikal, A.A. et al. 2000). 


\section{Chromophore Maturation Kinetics}

A crucial characteristic, which determines whether an FP works as a sufficient fusion partner with another protein, is the time a chromophore needs to maturate at physiological temperature. A maturation half time of 1 to 2 hours is sufficient to label most structures and proteins. However, detecting proteins with short lifetimes and early promoter activity by FP expression assumes the usage of FPs exhibiting faster maturation (Chudakov, D.M. et al. 2010). An example for a mutation, which decreases the maturation half time (beside other mentioned effects on FP characteristics - see Chapter II.2), is S65T in avGFP.

\section{Photostability}

Increasing the photostability of FPs is a major issue in protein generation due to the fact that it limits the amount of detectable photons emitted by an FP. However, the non-linear dependence of photostability and excitation light intensities (see Chapter II.1.a) makes screening for more photostable FPs challenging. TagRFP-T and mOrange2 are two examples for improved photostable variants in comparison to their progenitor proteins TagRFP and mOrange (Shaner, N.C. et al. 2008).

Depending on the application, the relevance of the different characteristics is different. Further, the optimization of one property is often at the expense of another characteristic. Hence the generation process of a new optimized FP mostly consists of balancing the advantages and disadvantages of mutations and is guided by the requirements of the specific application.

\section{e. Photoactivatable and Photoconvertable Fluorescent Proteins}

Besides the classical FPs, in the last few years, a new subgroup of FPs has been investigated which can be irreversible switched between two different fluorescent states. This switching is driven by light different from the excitation wavelength. These FPs can be separated into two groups: photoactivatable (Patterson, G.H. 
and Lippincott-Schwartz, J. 2002; Verkhusha, V.V. and Sorkin, A. 2005) and photoconvertable FPs (Mizuno, H. et al. 2003; Gurskaya, N.G. et al. 2006).

\section{Photoactivatable FPs}

Photoactivatable FPs are non fluorescent in their initial state. Upon irradiation with light of short wavelengths, they are converted to a state which can fluoresce by excitation with light of longer wavelength. One member of this protein group is PA-GFP (Patterson, G.H. and Lippincott-Schwartz, J. 2002). It can be switched with light of $\sim 400 \mathrm{~nm}$ into the fluorescent state, in which it can be excited at $\sim 488$ $\mathrm{nm}$ and emits light at $512 \mathrm{~nm}$. The structural basis of this photoactivation process is similar to the one appearing in avGFP when it is irreversibly converted from the neutral to the anionic state by the decarboxylation of Glu222 (see chapter II.2.b). The neutral form representing the non fluorescent state can be excited by $\sim 400$ $\mathrm{nm}$ leading to the decarboxylation of Glu222 (Figure II.12.A). This results in a

A

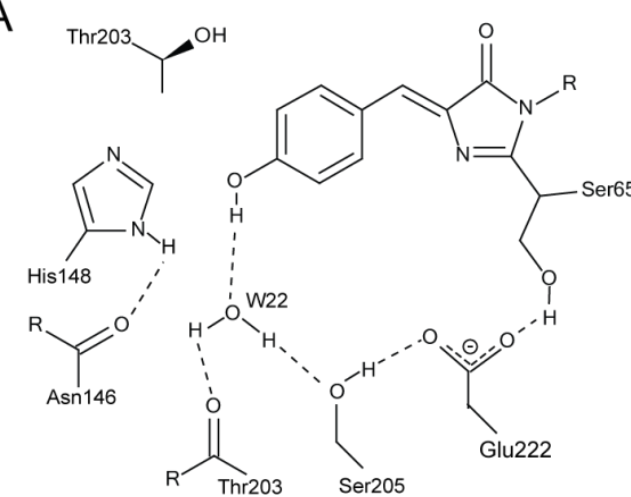

B

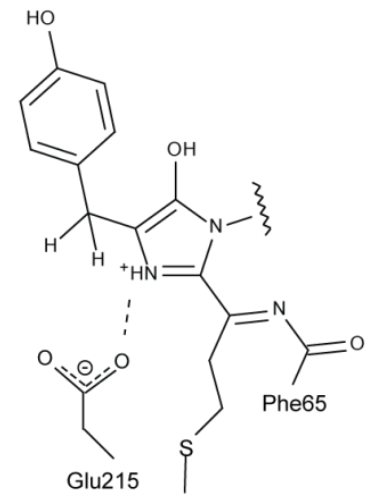

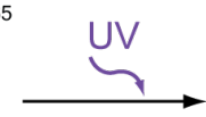
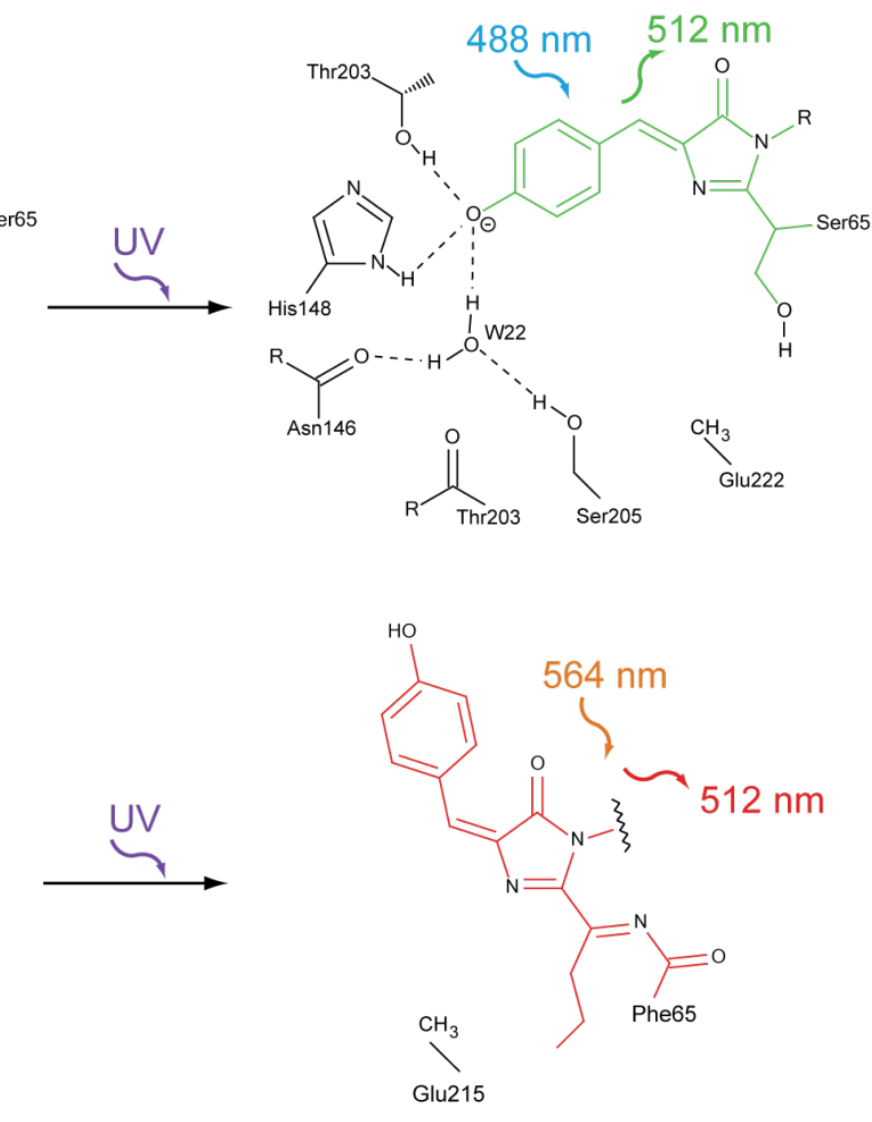

Figure II.12: Structural basis of photoactivation. (A) Excitation of PA-GFP leads to a decarboxylation of Glu222 arresting the chromophore in the anionic state. (B) Excitation of PAmCherry leads to an oxidation of the Tyr67 Ca-C $\beta$ bond elongating the m-electron system and resulting in the functional red chromophore. 
rearrangement of the hydrogen network stabilizing the anionic form, which exhibits fluorescence at $512 \mathrm{~nm}$.

Examples for red fluorescent photoactivatable FPs are PA-mRFP1 (Verkhusha, V.V. and Sorkin, A. 2005), PATagRFP (Subach, F.V. et al. 2010) and KFP1 (Chudakov, D.M. et al. 2003). The structural basis for the activation reaction in red variants was investigated for PA-mCherry1 (Subach, F.V. et al. 2009). It is suggested that in the non fluorescent state the $C \alpha$ and $C \beta$ of the chromophore amino acid Tyr67 are connected by a single bond, which interrupts the m-electron system between the $p$-hydroxyphenyl ring and the imidazole ring of the DsRedtype chromophore. Irradiation with UV light leads to a decarboxylation of Glu215 followed by the oxidation of this $\mathrm{C} \alpha-\mathrm{C} \beta$ bond, which results in the final red fluorescent chromophore (Figure II.12.B).

\section{Photoconvertable FPs}

The group of photoconvertable FPs describes proteins which can be irreversibly switched from one fluorescence color to another. This color conversion is induced by light. The protein Kaede can be exited by $508 \mathrm{~nm}$ and fluoresces at $518 \mathrm{~nm}$. Irradiation with light of $\sim 365 \mathrm{~nm}$ induces a photoconversion leading to a red shifted state that fluoresces at $582 \mathrm{~nm}$ upon excitation with $572 \mathrm{~nm}$ light (Ando, R. et al. 2002). The UV induced conversion is based on a $\beta$-elimination reaction leading to a break of the protein backbone. This elongates the $\pi-$ electron system to the histidine residue of the first chromophore amino acid (Figure II.13). Further, green to red converting FPs are EosFP (Wiedenmann, J.
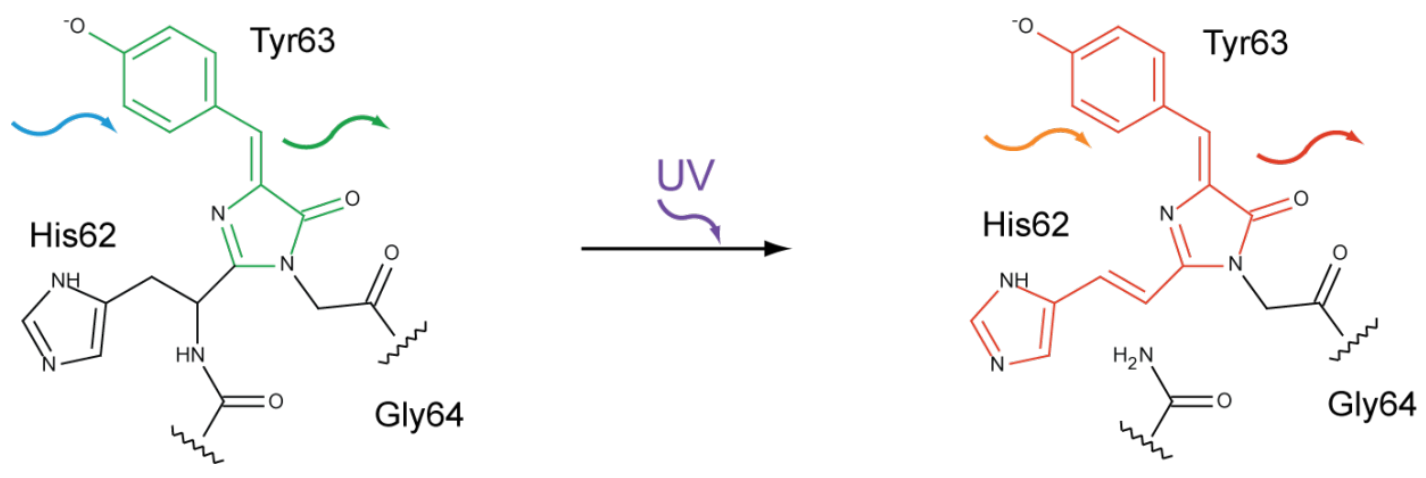

Figure II.13: Structural basis of green to red photoconversion. Exciting a photoconvertable FP with UV light leads to a photoconversion from a green to a red chromophore type by a $\beta$ elimination reaction causing a chain break of the protein backbone. 
et al. 2004) and Dendra (Gurskaya, N.G. et al. 2006). For EosFP it was shown that the basic photoconversion reaction is the same as in Kaede (Nienhaus, K. et al. 2005; Nienhaus, G.U. et al. 2006). Interestingly, it was shown that also avGFP variants can undergo a green to red conversion under low oxygen conditions (Elowitz, M.B. et al. 1997; Sawin, K.E. and Nurse, P. 1997; Jakobs, S. et al. 2003).

\section{Applications of Photoactivatable and Photoconvertable FPs}

The possibility to control the switching between two fluorescent states by light makes photoactivatable and photoconvertable FPs useful markers for a variety of applications such as protein tracking over time (Patterson, G.H. and LippincottSchwartz, J. 2002; Chudakov, D.M. et al. 2004) and especially single molecule switching methods in super-resolution microscopy (Betzig, E. et al. 2006). By applying a short UV light pulse to the probe, just a small number of the photoactivatable/photoconvertable FPs are switched, allowing single molecule detection. Afterwards, the switched molecules are bleached. The repetition of this procedure enables the reconstruction of a super-resolution microscopy image (see also chapter II.1.c). In contrast to this, photoactivatable and photoconvertable FPs can not be utilized for RESOLFT-type super-resolution microscopy based on the fact that this method requires reversible switching of the fluorophore. Here, another class of FPs comes into play: the Reversibly Switchable Fluorescent Proteins (RSFPs).

\section{f. Reversibly Switchable Fluorescent Proteins}

Reversibly Switchable Fluorescent Proteins (RSFPs) are a subgroup of FPs which can be switched between a fluorescent and a non-fluorescent state. This is driven by light of two different wavelengths. The switching of these proteins can be repeated several times, the switching is thus reversible. The first discovered RSFP was asFP595 isolated from the sea anemone Anemonia sulcata (Lukyanov, K.A. et al. 2000). Upon irradiation with green light of $\sim 570 \mathrm{~nm}$ asFP595 converts to the fluorescent state. The same wavelength also excites the protein to red fluorescence emission. Blue light of $\sim 450 \mathrm{~nm}$ switches the protein back into the non-fluorescent state (Figure II.14.A). This type of switching is 
classified as so called a positive switching, describing switching modes in which the excitation and the on-switching wavelengths are identical. In contrast, a negative switching mode is defined as switching in which excitation and offswitching wavelengths are

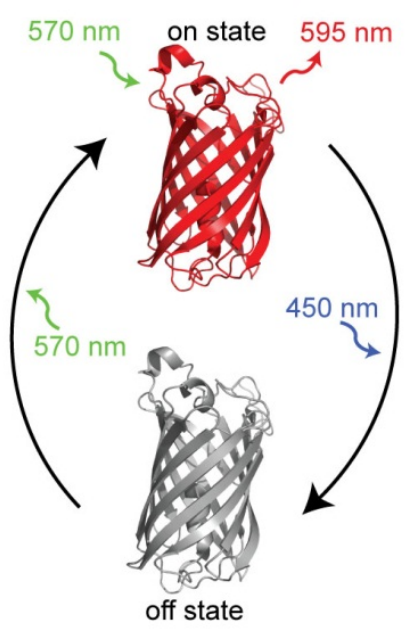

b

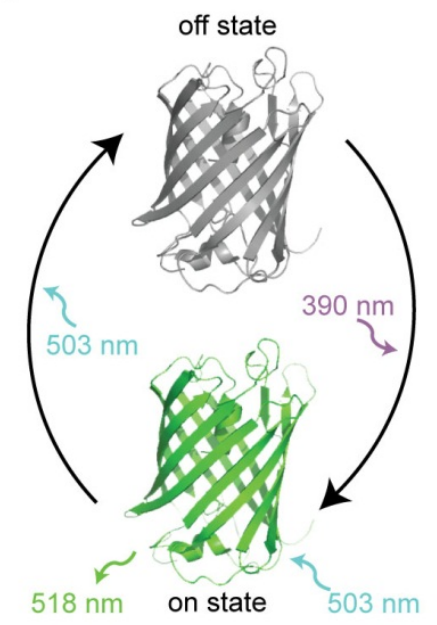
the same. An example for a negative switching RSFP is Dronpa (Ando, R. et al. 2004). It was discovered
Figure II.14: Switching modes in RSFPs. The excitation wavelength of the positive switching asFP595 switches the protein in the on state (a), while excitation wavelength of the negative switching Dronpa switches the protein into the off state (b).

during monomerisation experiments of FPs from Pectiniidae sp.. Dronpa can be excited with $488 \mathrm{~nm}$ light leading to green fluorescence. The same light switches the protein into the non-fluorescent state. Light of $405 \mathrm{~nm}$ switches Dronpa back into the fluorescent state (Figure II.14.B).

The X-ray structures of both proteins in the fluorescent on- and the nonfluorescent off-states revealed the structural key event in RSFP switching. The on-state chromophores of the RSFPs exhibit a cis conformation. Upon switching to the non-fluorescent state, the chromophores isomerize into the trans conformation (Figure II.15)(Andresen, M. et al. 2005; Andresen, M. et al. 2007; Stiel, A.C. et al. 2007). The switching is accompanied by a change in the chromophoric protonation state (Schuettrigkeit, T. et al. 2006; Schäfer, L.V. et al. 2007). In Dronpa the on-state cis chromophore is completely deprotonated resulting in a single absorption

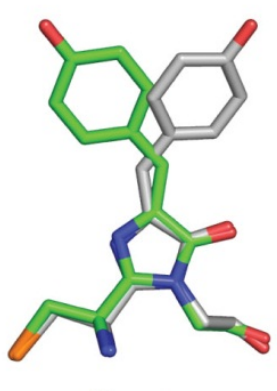

Dronpa

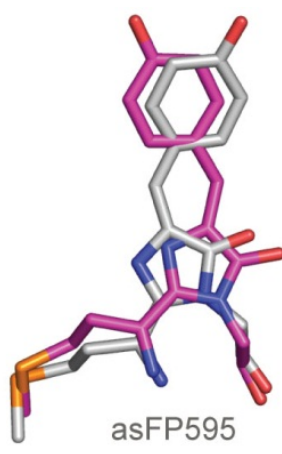

Figure II.15: Chromophore structures of Dronpa and asFP595. In the fluorescent on state, the chromophores adopts a cis conformation (green and red). Switching into the non fluorescent state leads to an isomerization of the chromophores into a trans conformation (gray). 
peak at $\sim 500 \mathrm{~nm}$. In contrast, the off-state chromophore in the trans conformation is completely protonated. The resulting single absorption peak at $\sim 390 \mathrm{~nm}$ enables the excitation with UV light to switch the RSFP back into the fluorescent state.

The trans conformation itself appearing in the off-state of an RSFP may not be the only reason why the protein is non-fluorescent. This is based on the fact that a cis conformation is indeed favored in FPs but never the less some FPs are known to fluoresce in the trans state (see Chapter II.2.b)(Petersen, J. et al. 2003; Merzlyak, E.M. et al. 2007; Malashkevich, V.N. et al. 2010). Based on NMR studies, it was suggested that a high flexibility of the protonated chromophore may prefer a thermal deexcitation (Mizuno, H. et al. 2008). However, resent studies revealed that a non-coplanar conformation of the chromophore in the trans state is the most likely reason for not being fluorescent (Brakemann, T. et al. 2010).

Based on the knowledge of the structural basis of switching, a variety of optimized and new RSFPs was generated. Mutations of Met159 in Dronpa led to a faster switching variant. One example is rsFastLime (Dronpa M159T), a bright RSFP with a 50 fold decreased halftime in off switching (Stiel, A.C. et al. 2007). Further mutations in rsFastLime yielded Padron, which exhibits a reversed positive switching mode. In the off-state Padron is completely deprotonated. In the fluorescent state it is partially protonated.

With the generation of positive switching rsCherry and negative switching rsCherryRev, the first monomeric red fluorescent RSFPs were established (Stiel, A.C. et al. 2008). Both proteins were designed on the basis of mCherry. This shows that classical FPs can be converted into RSFPs by mutagenesis. In 2010 a further red emitting RSFP called rsTagRFP, a variant derived from TagRFP, was developed (Subach, F.V. et al. 2010).

The protein IrisFP represents a unique type of RSFP, which was derived from EosFP (Adam, V. et al. 2008). On one hand it can be converted from a green to a red fluorescent state. It can also be reversibly switched between an on-state and an off-state. Hence this protein demonstrates that photoconversion and reversible photoswitching can be combined in one protein. 


\section{Applications of Reversibly Switchable Fluorescent Proteins}

The unique properties of RSFPs make them preferable for a variety of applications. For example RSFPs exhibit a major advantage for the tracking of protein movements. A subset of proteins at a certain position can be switched on. Subsequently, these proteins can be tracked over time, so that differences in motility can be detected (Ando, R. et al. 2004; Chudakov, D.M. et al. 2010). In comparison to photoactivatable and photoconvertable FPs, the use of reversibly switchable proteins allows repeated measurements of the same movement. Hence, statistical noise can be reduced (Chudakov, D.M. et al. 2006).

Further applications using RSFPs as data storage media are often discussed (Ando, R. et al. 2004). Here, the two different fluorescent states can represent the " $0 \mathrm{~s}$ " and " $1 \mathrm{~s}$ " of the binary code. The reversibility in switching would make RSFPs rewritable data storage media. In proof-of-principle experiments storing data in 2D using a protein layer and in 3D using protein crystals was demonstrated (Adam, V. et al. 2010). However, the strong switching fatigue and the relatively low switching speeds of known RSFPs strongly limit their usability for data storage applications.

A major field of application utilizing RSFP is super-resolution microscopy. The basic requirements for these fluorescence microscopy approaches is the possibility to discern between two different fluorescent states (see also chapter II.1.c), which is given by the intrinsic reversibly switching of RSFPs. In contrast to photoactivatable and photoconvertable FPs, which have to be bleached after detecting them (see also chapter II.2.e), RSFPs can be detected several times in SMS microscopy approaches (Flors, C. et al. 2007; Andresen, M. et al. 2008; Stiel, A.C. et al. 2008). This fact extends the usability of SMS microscopy for time lapse experiments.

RSFPs are the only subgroup of FPs providing an intrinsic reversible switching. This makes them interesting candidates for use in RESOLFT microscopy. Furthermore, in contrast to STED microscopy, RSFPs can be effectively switched with much lower intensities. Hence, RSFP-based RESOLFT should be favorable for live cell applications.

However, up to now, using RSFPs in RESOLFT approaches was strongly limited to proof-of-principle experiments, like for example imaging of small structures 
stained with highly concentrated, purified protein (Hofmann, M. et al. 2005; Dedecker, P. et al. 2007). Biological imaging remained unviable. The reason for this is the lack of RSFPs providing all the properties needed for such a microscopic approach. The switching fatigue of known RSFPs is too strong, meaning that they can not undergo the required number of switching cycles before being bleached (see also chapter II.1.c). A further factor making RSFPRESOLFT challenging is the relatively slow switching of known RSFPs. During imaging a whole switching cycle on each scanning position has to be performed. The use of slow switching RSFPs would lead to very long imaging times which is incompatible with live cell approaches. A further important requirement for RESOLFT approaches is a low residual fluorescence in the off-state. A low contrast would lead to destruction of the RESOLFT switching pattern. In conclusion, new RSFPs providing low switching fatigue, high switching contrast and fast switching speeds are needed to make RSFP-RESOLFT a working super-resolution microscopy method to study live cell processes. 


\section{Aims}

This work focuses on the generation of novel RSFPs enabling a RESOLFT microscopy approach to extend the spectrum of super-resolution microscopy techniques with a viable technique for live cell analysis on the nanoscale and minimizing the risk of phototoxic effects by applying just low levels of light. For use in RESOLFT these RSFPs must possess the following characteristics:

High brightness, a truly monomeric nature, appropriate folding characteristics, and most notably, low switching fatigue and fast switching kinetics. To realize these aims, this work was executed in the following sequence:

1. Establishment of a screening setup that characterizes all crucial protein properties using high light intensities, thereby identifying improved RSFP variants.

2. Generation of new enhanced RSFP variants by directed and random mutagenesis supported by the established screening system.

3. Establishment of a RESOLFT-type microscopy approach for live cell analysis by utilizing the new RSFPs.

4. Investigation of the new RSFPs for their applicability to other technologies, mainly focusing on data storage. 


\section{Results}

\section{Generation and Establishment of a Screening System to Study RSFP Characteristics}

To generate new RSFPs a screening system is needed, which allows an automated search for enhanced variants in large protein collections. To minimize the appearance of false positives, it is necessary that the system characterizes all protein properties of interest as precisely as possible. Furthermore, to maximize throughput, fast screening speeds are desirable. A fast way to analyze and sort for specific FP variants is to express them in E. coli and screen these cells by FACS (Fluorescene Activated Cell Sorting) machines. However, the interaction time of a cell analyzed by FACS lies in the $\mu$ s time range. This is too short to detect the switching kinetics of RSFPs, a crucial characteristic which determines the suitability of an RSFP for RESOLFT applications. Hence a custom built screening setup was designed. This setup determines all relevant protein characteristics, including brightness, residual fluorescence in the off-state, switching kinetics, bleaching and switching fatigue.

The screening system was designed by customizing an automated microscope setup. E. coli colonies growing on agar plates and expressing different FP variants were identified by their fluorescence emission while scanning over a plate (Figure IV.1.A). To perform and detect switching, a variable light illumination protocol was performed on each colony, while reading out fluorescence.

Bleaching and switching fatigue, the two most crucial characteristics for RESOLFT microscopy, strongly depend on the applied light intensities (see chapter II.1.a). Therefore, it was important to screen under the same light intensities as required for RESOLFT microscopy. These intensities in the $\mathrm{kW} / \mathrm{cm}^{2}$ range were achieved by the utilization of lasers as light sources, in combination with a $20 \times$ objective lens. Two lasers provided light of wavelengths $405 \mathrm{~nm}$ (20 $\mathrm{mW})$; and $491 \mathrm{~nm}(50 \mathrm{~mW})$, and $532 \mathrm{~nm}(50 \mathrm{~mW})$, respectively (Figure IV.1.B). The intensities of the different wavelengths were controlled by an acousto-optic transmission filter (AOTF) and an acousto-optic modulator (AOM). The beams were combined and coupled into an optical fiber. This turned out to be favorable, 
A
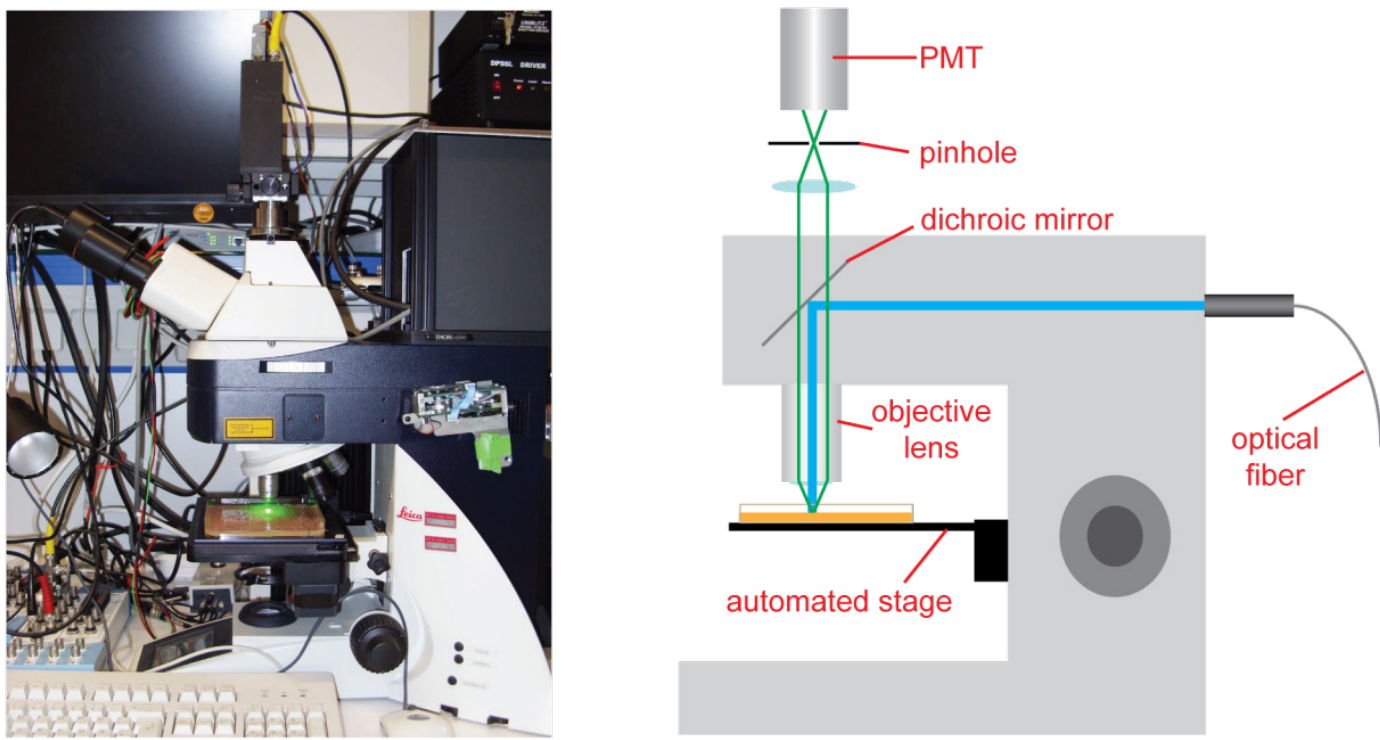

B

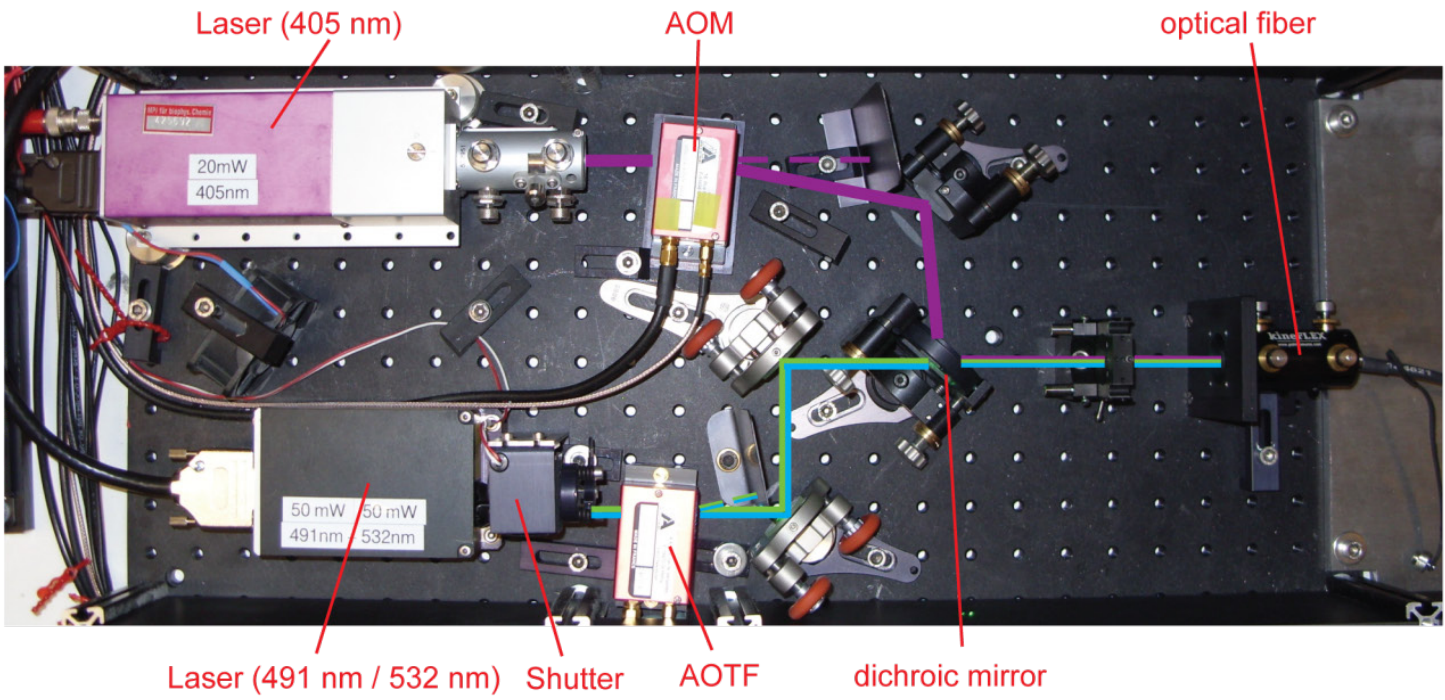

Figure IV.1: A laser-based screening setup. (A) An automated microscope is used to search and characterize E. coli colonies expressing different RSFP variants. (B) To reach focus intensities in the $\mathrm{kW} / \mathrm{cm}^{2}$ range a $20 \mathrm{~mW} 405 \mathrm{~nm}$ laser and a dual color $50 \mathrm{~mW} 491 \mathrm{~nm} / 532 \mathrm{~nm}$ laser were used as light sources.

because it ensures a stable overlay of all three beams, thereby eliminating the potential falsification of data caused by independent drift of the different beams during long time screenings. The light was coupled into the microscope stand, reflected by a dichroic mirror, and focused onto the $E$. coli sample by the objective lens (Figure IV.1.A). This assembly results in foci with a diameter of 1-2 $\mu \mathrm{m}$ (Table IV.1). These sizes turned out to be optimal for this kind of screening approach, because they were small enough to reach proper intensities, while being large enough to probe several cells at a time. Foci, much smaller than a single $E$. coli cell, resulted in data artifacts due to diffusion effects inside 
the cell. The emission light was collected by the objective lens and passed through the dichroic mirror. Finally, the emitted light was detected by a PMT.

E. coli colonies are relatively thick samples. Stray light of FPs above and below the focal plane can falsify bleaching and switching data. This problem was minimized by adding a confocal pinhole of diameter $0.1 \mathrm{~mm}$ in the detection path.

Table IV.1: Foci diameter and maximum achievable light intensities of the custom built screening setup.

\begin{tabular}{cccc} 
Laser & $\begin{array}{c}\text { focus diameter } \\
{[\mu \mathrm{m}]}\end{array}$ & $\begin{array}{c}\text { maximum Intensity } \\
\text { using AHF 495DCXR /488LP } \\
\text { filter cube } \\
{\left[\mathrm{kW} / \mathrm{cm}^{2}\right]}\end{array}$ & $\begin{array}{c}\text { maximum Intensity } \\
\text { using AHF 570DCXR /572LP } \\
\text { filter cube } \\
{\left[\mathrm{kW} / \mathrm{cm}^{2}\right]}\end{array}$ \\
\hline $405 \mathrm{~nm}$ & 1.6 & $\sim 110$ & $\sim 110$ \\
$491 \mathrm{~nm}$ & 2 & $\sim 270$ & $\sim 400$ \\
$532 \mathrm{~nm}$ & 2.1 & $\sim$ &
\end{tabular}

The whole system, including light intensities, stage, and PMT voltage, was controlled by a LabView-based software (programmed by Dr. Christian Eggeling). The screening software protocol was divided into three major steps. In the first step the agar plate was scanned for colonies. These were detected by fluorescence exceeding a defined threshold value. This fluorescence detection step was performed with low light intensities to exclude an induction of switching or bleaching processes. Once a colony was detected, step 2, an autofocus function to determine the optimal focal plane, was performed. It turned out, that this step was essential because of the small focal volumes. The fluorescence intensity at several z-positions were determined using the same light intensity as utilized in step 1. The position of highest fluorescence intensity defined the optimal focal plane. Alternative autofocus schemes, which detected scattered light instead of fluorescence, were found to be less precise. In the final step, the protein characteristics were determined by applying adaptable illumination sequences to the sample. The light intensities used in this third step were in the $\mathrm{kW} / \mathrm{cm}^{2}$ range to ensure illumination conditions comparable to those typical of RESOLFT microscopy. The use of higher intensities demanded the possibility to 
adapt the PMT voltage in step 3 because of the resulting higher fluorescence signals. It was established, that switching RSFPs four times was sufficient to determine bleaching and switching fatigue characteristics. These three program steps were repeated until the whole plate was screened. Approximately 2000 colonies were analyzed per plate. This typically took several hours strongly depending on the illumination sequence. For analysis and identification of enhanced protein variants the collected data were analyzed with a second program. The location of all analyzed colonies was recorded. Thus, all E. coli cells expressing proteins of interest could be retraced and picked.

The developed screening systems provided precise and reproducible data of all RESOLFT microscopy relevant RSFP properties. It represented a viable system to characterize RSFPs and screen for enhanced variants.

\section{Characterization and Comparison of commonly used RSFPs}

In the next step, the screening system was utilized to characterize the current most frequently used RSFPs to identify their respective limiting factors for RESOLFT (see chapter II.2.f). E. coli expressing the respective proteins were excited for fluorescence emission and switching by light of either $491 \mathrm{~nm}$ (Dronpa, rsFastLime, Padron) or $532 \mathrm{~nm}$ (rsCherryRev), both at an intensity of 20 $\mathrm{kW} / \mathrm{cm}^{2}$. By adding light of $405 \mathrm{~nm}\left(30 \mathrm{~kW} / \mathrm{cm}^{2}\right)$ proteins were switched into the other direction. Each protein was cycled 100 times.

The negative switching RSFP, Dronpa, showed strong switching fatigue. It underwent just $\sim 4$ cycles before it was bleached to half of its initial fluorescence (Figure IV.2). Also, the enhanced Dronpa variant, rsFastLime, allowed just $~ 35$ cycles before being bleached to $50 \%$ fluorescence. Strong switching fatigue was also determined for the positive switching variant Padron. It facilitated just 15 switching cycles. Furthermore, the residual fluorescence of Padron in the offstate of $15 \%$ is too high to exhibit a sufficient switching contrast for RESOLFT applications.

In contrast, the red fluorescent RSFP, rsCherryRev, a variant with a negative switching mode, seemed to exhibit no switching fatigue. Instead, it became 
brighter, due to a photoinduced structural change, which was already described before (Stiel, A.C. et al. 2008). However, also rsCherryRevs switching contrast of $5: 1$ of was not satisfactory (Figure IV.2).

Thus, none of the RSFPs analyzed met criterion required for their utilization in RESOLFT, either the switching fatigue was too strong and/or the switching contrast was too low.

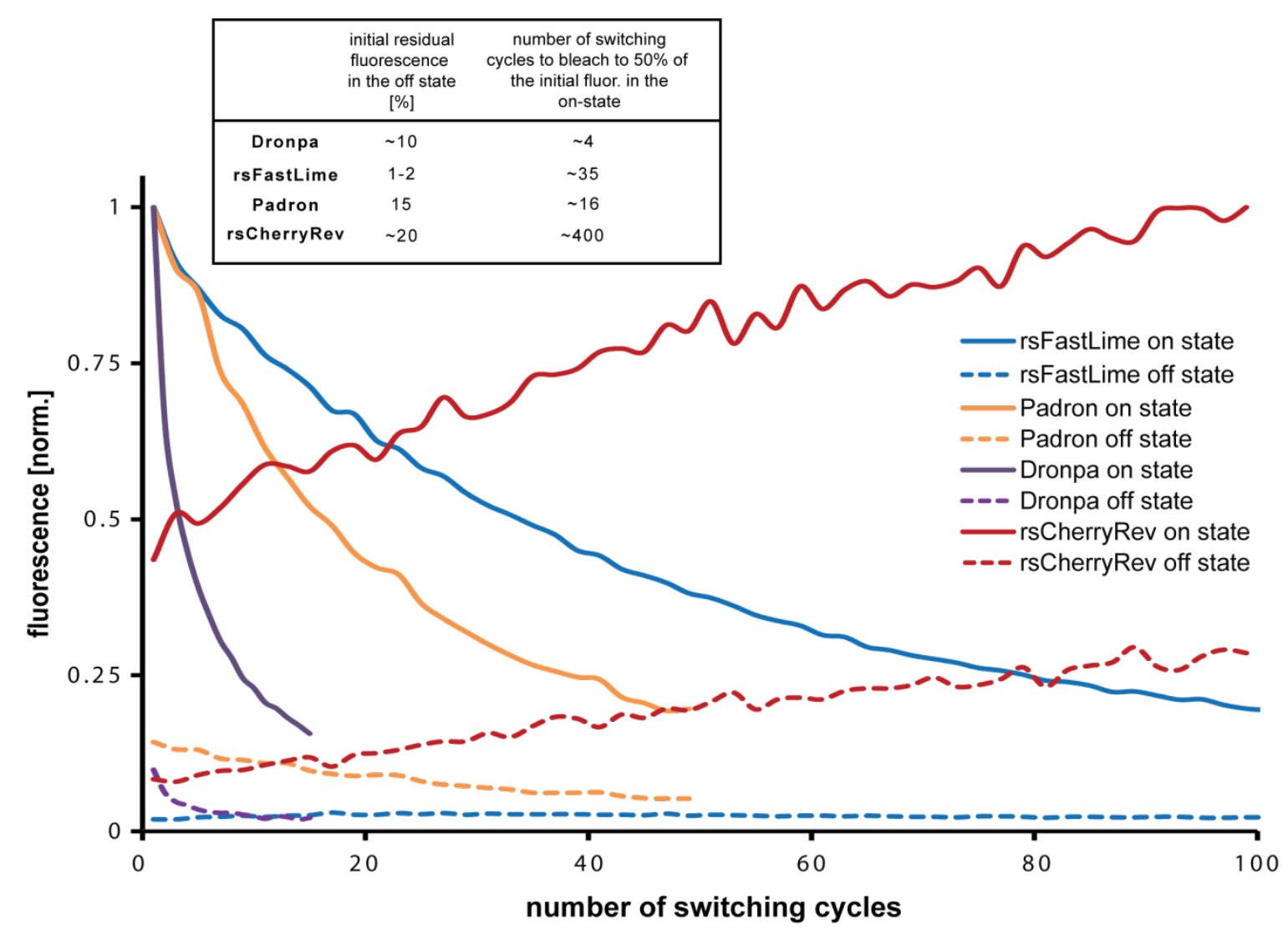

Figure IV.2: Switching characteristics of RSFPs. Plotted are the respective normalized fluorescence intensities in the on-state (solid lines) and the off-state (dashed lines) against the cycle number. Measurements were performed on living $E$. coli colonies expressing the indicated RSFP. The duration of the switching cycles were adjusted to the spectroscopic properties of the RSFPs. Care was taken to ensure that all proteins were completely switched, and the irradiation times kept as short as possible. For switching and fluorescence readout, colonies were constantly irradiated with light of wavelength $491 \mathrm{~nm}\left(\sim 20 \mathrm{~kW} / \mathrm{cm}^{2}\right)$ or, in the case of rsCherryRev, 532 $\mathrm{nm}\left(\sim 20 \mathrm{~kW} / \mathrm{cm}^{2}\right)$. For switching in the other direction, light of wavelength $405 \mathrm{~nm}\left(30 \mathrm{~kW} / \mathrm{cm}^{2}\right)$ was applied. (Inset) Residual fluorescence intensity in the off-state and the number of switching cycles until the fluorescence is bleached to $50 \%$. 


\section{Reversibly Switchable Enhanced Green}

\section{Fluorescent Protein (rsEGFP)}

To generate RSFPs exhibiting a low switching fatigue, a high photostability, and fast switching kinetics, a protein is needed which can be used as a starting point. One of the best characterized and widely used FP is EGFP. It shows fast folding, can be fused easily to a wide variety of different structures, exhibits high brightness, and most notably, it features high photostability. Therefore, this protein was modified to generate new RSFPs exhibiting all relevant characteristics needed for RESOLFT applications.

\section{a. Generation of rsEGFP}

To obtain a switchable EGFP, numerous variants of this protein were generated by site-directed and random mutagenesis followed by screening of these protein collections. Light driven switching of RSFPs is facilitated by an isomerization of the chromophore accompanied by a change of its protonation state (see chapter II.2.f). Hence, a main focus lay on the mutagenesis of amino acids in close proximity of the chromophore which may directly influence the possibility for isomerization. These amino acids were determined by using the available X-ray structure of avGFP (Ormo, M. et al. 1996). Each of these amino acids was exchanged against all other possible amino acids by site-directed mutagenesis. Furthermore, all relevant amino acids identified by random mutagenesis were exchanged by site-directed mutagenesis to determine, which amino acid residue exhibited the optimal effect at this position. Care was taken to maintain the key mutations, S65T and F64L of EGFP. To ensure that all generated variants act as true monomers, the mutation A206K was introduced (Zacharias, D.A. et al. 2002). In addition, an exchange to charged amino acids was avoided, if they were oriented to the outside of the protein.

During the whole generation process $>50,000$ different variants were screened. It turned out that a single mutation, Q69L, makes mEGFP (= EGFP-A206K) switchable. However, the resulting contrast between the on- and the off-state, of 2:1, was insufficient. Adding the mutations V150A, V163S, and S205N created an RSFP providing high brightness, fast switching, low switching fatigue, and a 
small value of residual fluorescence in the off-state. This variant was named rsEGFP (reversibly switchable EGFP)(Figure IV.3).

EGFP MVSKGEELFTGVVPILVELDGDVNGHKFSVSGEGEGDATYGKLTLKFICTTGKLPVPW rsEGFP MVSKGEELFTGVVPILVELDGDVNGHKFSVSGEGEGDATYGKLTLKFICTTGKLPVPW

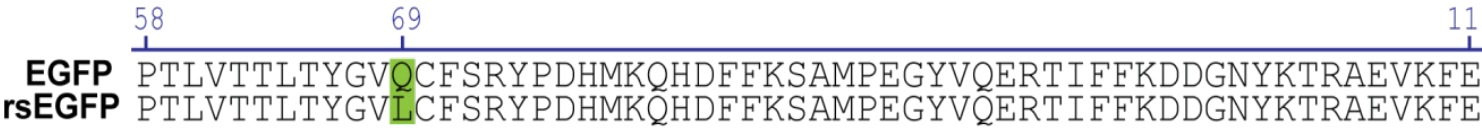

EGFP \begin{tabular}{lccc}
116 & 150 & 163 & 173 \\
\cline { 3 - 4 } & 1 & 1 & 1
\end{tabular} rsEGFP GDTLVNRIELKGIDFKEDGNI LGHKLEYNYNSHNAY IMADKQKNGIKSNFKIRHNIED

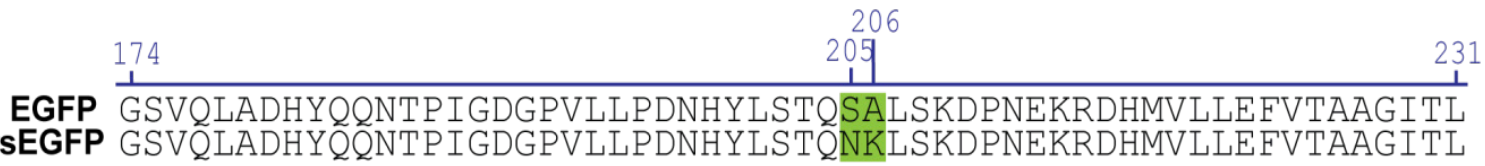
$\underset{\text { EGFP }}{\stackrel{232}{\text { GMDELYK }}} \stackrel{238}{\text { rsEGFP GMDELYK }}$

Figure IV.3: Amino acid sequence of EGFP and rsEGFP. Differences are highlighted in green.

\section{b. Determination of rsEGFP Characteristics}

rsEGFP adopts a bright fluorescent state at equilibrium, with a quantum yield $\Phi_{F L}$ of 0.36 and an extinction coefficient $\varepsilon$ of $47,000 \mathrm{M}^{-1} \mathrm{~cm}^{-1}$. Based on these data a relative brightness of $\sim 60 \%$ compared to EGFP was calculated (Table IV.2). The on-state absorption spectrum showed a single peak at $491 \mathrm{~nm}$, evidencing a chromophore in the deprotonated form. Excitation into this band yielded in a fluorescence peaking at $510 \mathrm{~nm}$ (Figure IV.4.A), but simultaneously switched the protein into a non-fluorescent state with a single absorption peak at $396 \mathrm{~nm}$ (Figure IV.4.B). This $396 \mathrm{~nm}$ peak indicated the presence of a protonated

Table IV.2: Properties of EGFP, rsEGFP and Dronpa. ( ${ }^{*}($ Patterson, G.H. et al. 1997) ${ }^{* *}($ Andresen, M. et al. 2008))

\begin{tabular}{|c|c|c|c|c|c|c|c|c|c|}
\hline & $\begin{array}{c}\text { extinction } \\
\text { coefficient } \\
(\varepsilon) \\
{\left[\mathrm{M}^{-1} \mathrm{~cm}^{-1}\right]}\end{array}$ & $\begin{array}{l}\text { fluorescence } \\
\text { quantum } \\
\text { yield } \\
\left(\Phi_{\mathrm{FL}}\right)\end{array}$ & $\begin{array}{c}\text { excitation } \\
\text { maximum } \\
\text { on-state } \\
{[\mathrm{nm}]}\end{array}$ & $\begin{array}{l}\text { single } \\
\text { molecule } \\
\text { brightness } \\
\text { [norm.] }\end{array}$ & $\begin{array}{c}\text { emission } \\
\text { maximum } \\
{[\mathrm{nm}]}\end{array}$ & $\begin{array}{c}\text { absorption } \\
\text { maximum } \\
\text { off-state } \\
{[\mathrm{nm}]}\end{array}$ & $\begin{array}{c}t_{1 / 2} \\
\text { on switching } \\
\left(2 \mathrm{~kW} / \mathrm{cm}^{2} \text { at } 405 \mathrm{~nm}\right) \\
{[\mu \mathrm{s}]}\end{array}$ & $\begin{array}{c}\mathrm{t}_{1 / 2} \\
\text { off switching } \\
\left(0.6 \mathrm{~kW} / \mathrm{cm}^{2} \text { at } 491 \mathrm{~nm}\right) \\
{[\mathrm{ms}]}\end{array}$ & $\begin{array}{c}\text { number of switching } \\
\text { cycles to bleach to } 50 \% \text { of } \\
\text { the initial fluor. in the on-state } \\
\left(2 \mathrm{~kW} / \mathrm{cm}^{2} \text { at } 405 \mathrm{~nm}\right. \\
\left.0.6 \mathrm{~kW} / \mathrm{cm}^{2} \text { at } 491 \mathrm{~nm}\right)^{3}\end{array}$ \\
\hline EGFP & $53.000^{*}$ & $0.6^{*}$ & 489 & 1 & 509 & n.a. & n.a. & n.a. & n.a. \\
\hline rsEGFP & 47.000 & 0.36 & 493 & 0.6 & 510 & 396 & $<20$ & $\sim 1$ & $\sim 1.200$ \\
\hline Dronpa & $125.000^{* *}$ & $0.68^{\star *}$ & 503 & 1.5 & 522 & 392 & $\sim 50$ & $\sim 16$ & $\sim 7$ \\
\hline
\end{tabular}

n.a.: not applicable 

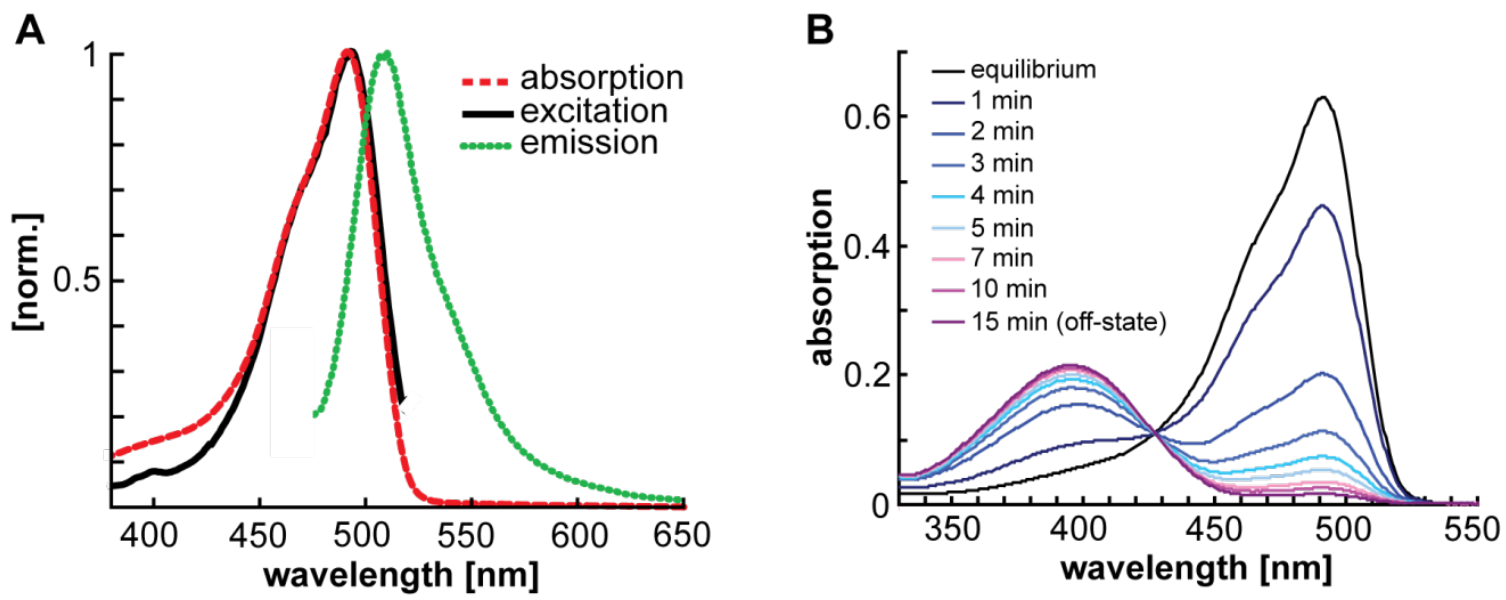

Figure IV.4:Spectral properties of rsEGFP. (A) Absorption, excitation, and emission spectra of rsEGFP in the fluorscent equilibrium state. Spectra were determined using purified protein at $\mathrm{pH}$ 7. (B) Changes in the absorption spectrum of rsEGFP during switching from the equilibrium to the off-state. The spectra were taken after different times of illuminating a cuvette of rsEGFP protein solution with $488 \mathrm{~nm}$ light.

chromophore in the non-fluorescent state of rsEGFP. The residual fluorescence in the off-state compared to the on-state fluorescence was $1-2 \%$. Excitation of the off-state protein with light of $\sim 400 \mathrm{~nm}$ wavelength switched the protein back into the on-state. This switching cycle could be repeated. Hence the switching is reversible.

rsEGFP switched into the off-state exhibits a thermal relaxation back into the equilibrium state. This occurs with a halftime of $\sim 23 \mathrm{~min}$ at room temperature (Figure IV.5).

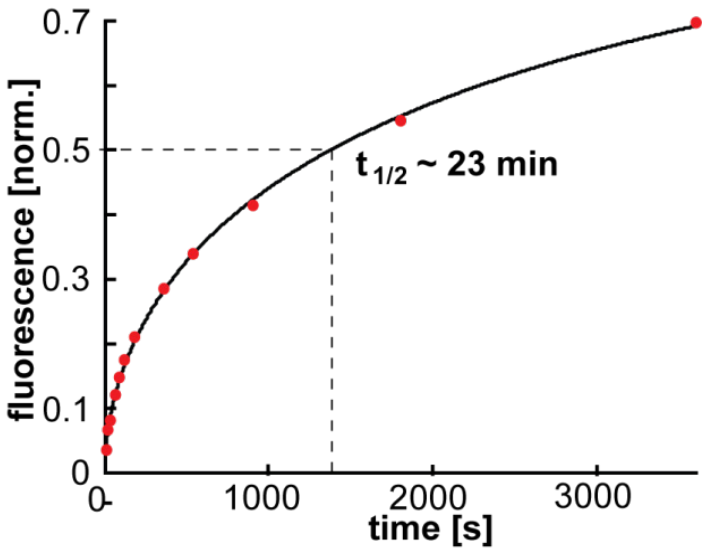

Figure IV.5: Relaxation kinetic of rsEGFP. Protein was immobilized in a PAA layer. A defined region was switched into off-state. Recovery was determined by detecting the increase of fluorescence over time. Readout excitation light intensity was kept as low as possible to reduce switching artifacts. The data were fitted with a stretched exponential function (stretching factor: $\sim 0.6$ ).

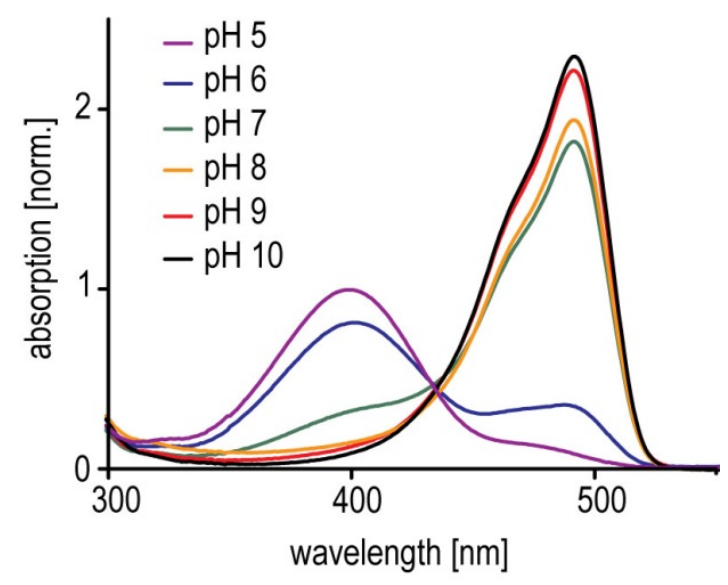

Figure IV.6: Absorption spectra of equilibrium-state rsEGFP at different $\mathrm{pH}$ values. Purified rsEGFP protein was diluted in various buffers $(\mathrm{pH} 5$ : $0.1 \mathrm{M}$ citrate buffer, $\mathrm{pH}$ 6, 7: 0.1M Tris- $\mathrm{HCl}$ buffer, $\mathrm{pH}$ 9, 10: $0.1 \mathrm{M}$ glycine buffer). 

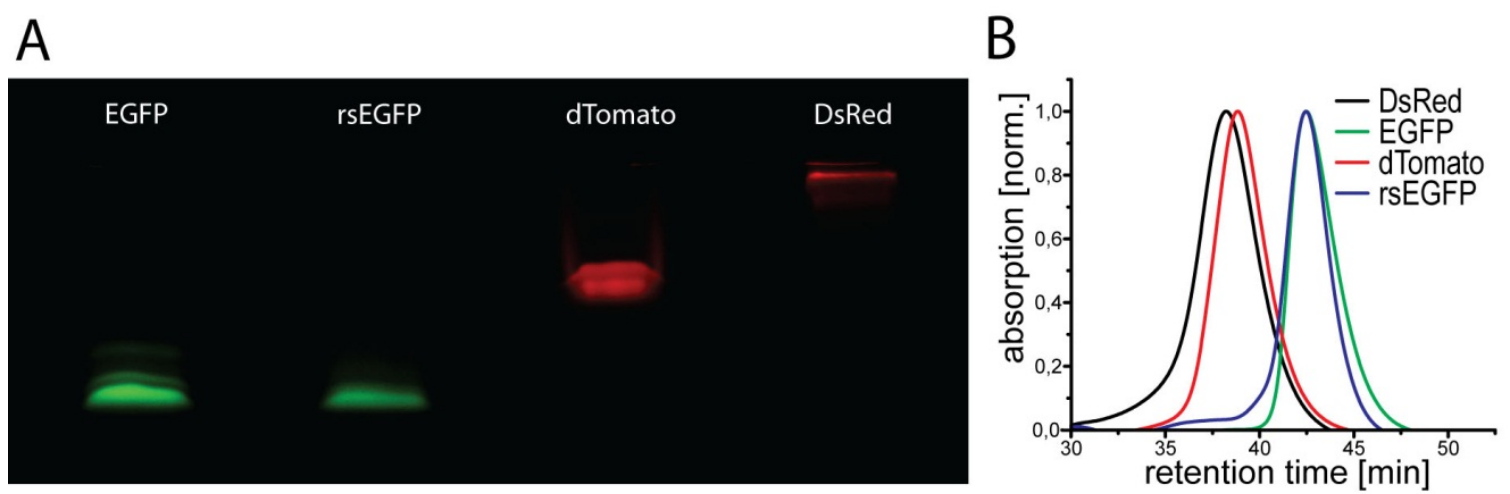

Figure IV.7: rsEGFP is a monomer. (A) Semi-native polyacrylamide gel electrophoresis of rsEGFP. Purified monomeric EGFP, dimeric dTomato, tetrameric DsRed, and rsEGFP were separated on a semi-native gel. Images were taken with a custom built gel documentation system. To detect green fluorescence (EGFP and rsEGFP) the gel was irradiated with light of 470 $\pm 5 \mathrm{~nm}$ and fluorescence was detected at $525 \pm 30 \mathrm{~nm}$. To detect red fluorescence (dTomato and DsRed) the gel was irradiated with light of $545 \pm 10 \mathrm{~nm}$ and fluorescence was detected at $617 \pm$ $37 \mathrm{~nm}$. Both images were overlaid and are represented in false colours. (B) Size separation chromatography at $4{ }^{\circ} \mathrm{C}$. Shown are chromatography runs of rsEGFP, monomeric EGFP, dimeric dTomato, and tetrameric DsRed.

The determination of rsEGFP's absorption spectra at different $\mathrm{pH}$ values revealed a peak transition from the $493 \mathrm{~nm}$ peak under alkaline and neutral conditions to the $396 \mathrm{~nm}$ peak under acidic conditions (Figure IV.6). This confirms a neutral chromophore in the off-state and an anionic chromophore in the on-state. A pKa of 6.5 was calculated.

To observe, whether rsEGFP is viable as an effective marker for live cell experiments, its oligomerization state and folding characteristics were determined. Semi-native polyacrylamide gel electrophoresis of rsEGFP and the marker proteins EGFP (monomer), dTomato (dimer) and dsRed (tetramer) at room temperatures demonstrated, that rsEGFP acts as a true monomer in vitro (Figure IV.7.A). Size separation chromatography at $4^{\circ} \mathrm{C}$ confirmed these results (Figure IV.7.B). The

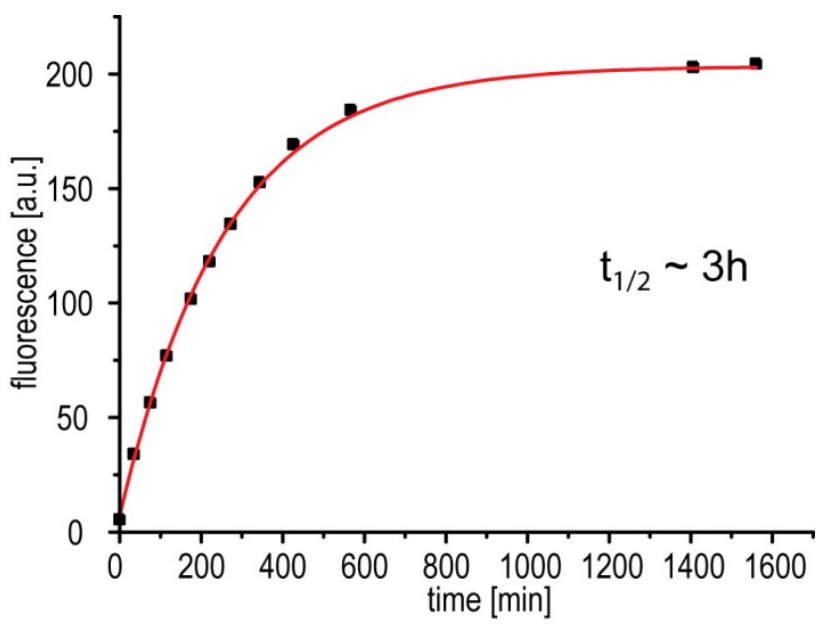
maturation halftime of rsEGFP at $37^{\circ} \mathrm{C}$ is $\sim 3$ hours (Figure IV.8).

Figure IV.8: Maturation kinetic of rsEGFP at $37^{\circ} \mathrm{C}$. 
A

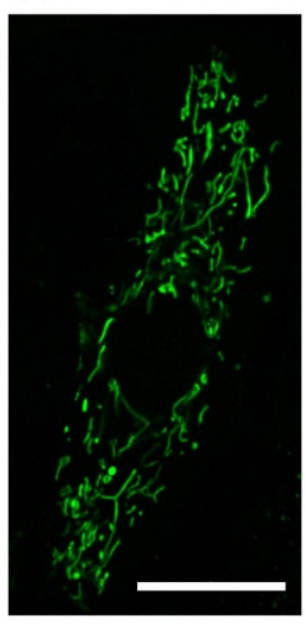

E

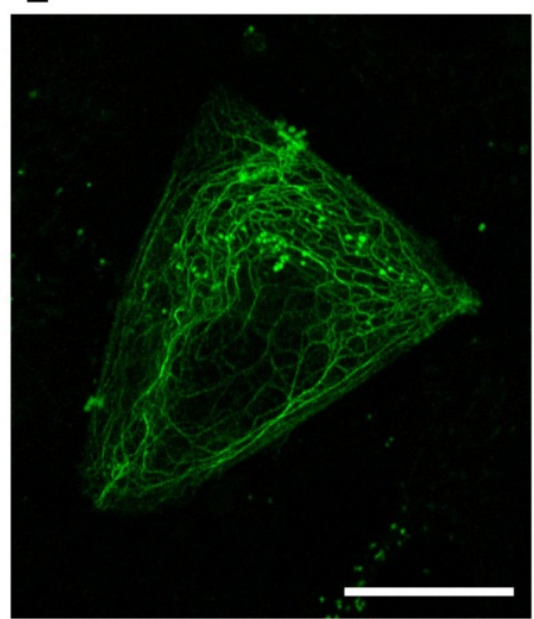

B

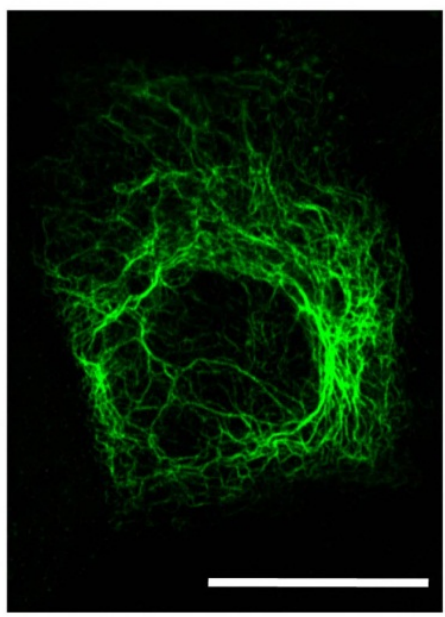

$\mathrm{F}$

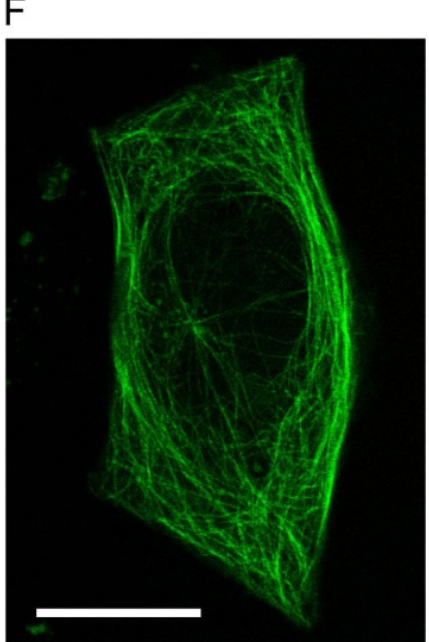

C

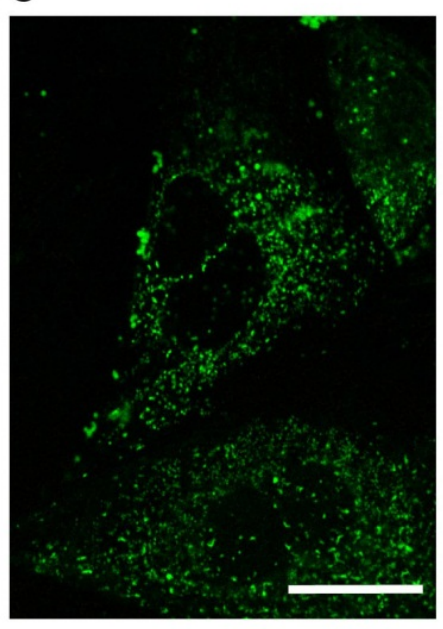

G

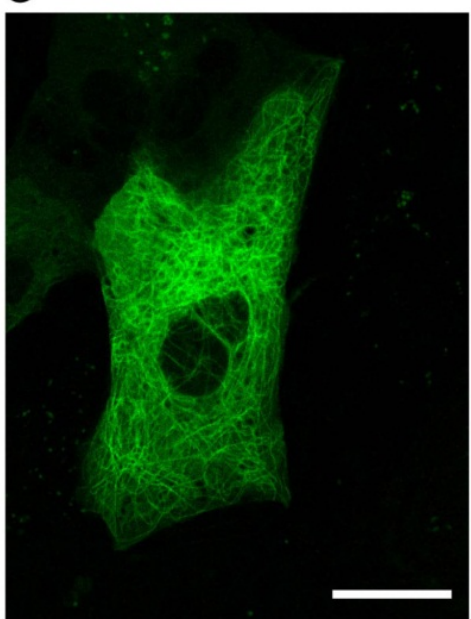

Figure IV.9: Expression of various functional rsEGFP fusion proteins in mammalian PtK2 cells. (A) Mito-rsEGFP, (B) Vimentin-rsEGFP, (C) Pex16-rsEGFP, (D) Histone H2B-rsEGFP, (E) Keratin19-rsEGFP, (F) rsEGFP-MAP2, and (G) rsEGFP- $\alpha-T u b u l i n$. Shown are single confocal sections of living cells. Fluorescence was excited by simultaneous irradiation with light of $488 \mathrm{~nm}$ and $405 \mathrm{~nm}$. Scale bars: $20 \mu \mathrm{m}$.

The monomeric nature and the adequate maturation time should enable the usage of rsEGFP as a fusion marker in living cells. To verify this assumption, various fusion constructs - a mitochondrial targeting sequence, Vimentin, Pex16, Histone H2B, Map2, Keratin19, and a-Tubulin - were cloned and expressed in PtK2 cells. After 48 hours of expression, cells were imaged. The imaged cells revealed typical morphologies of the respective constructs (Figure IV.9). Since histone $\mathrm{H} 2 \mathrm{~B}$ and $\alpha$-Tubulin are sensitive fusion partners requiring a monomeric and well maturing FP, these results prove, that rsEGFP can be used for functional fusion constructs. Expressed in PtK2 cells, rsEGFP exhibited the same fundamental spectral and switching characteristics as the purified protein. Hence, 

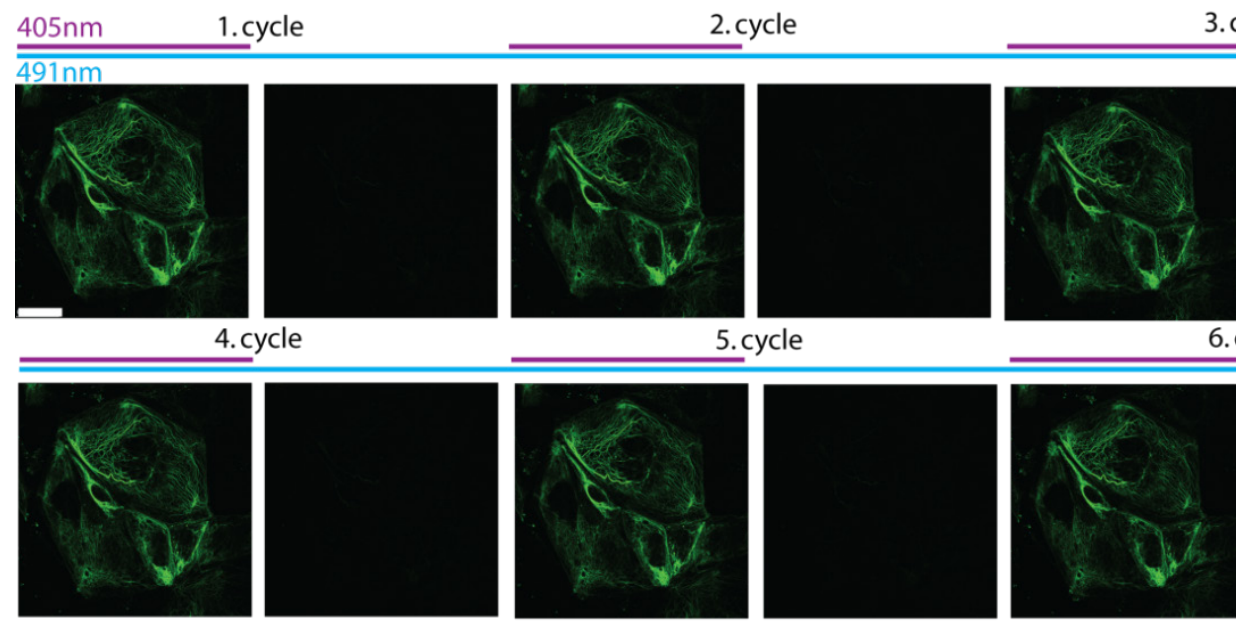

3. cycle
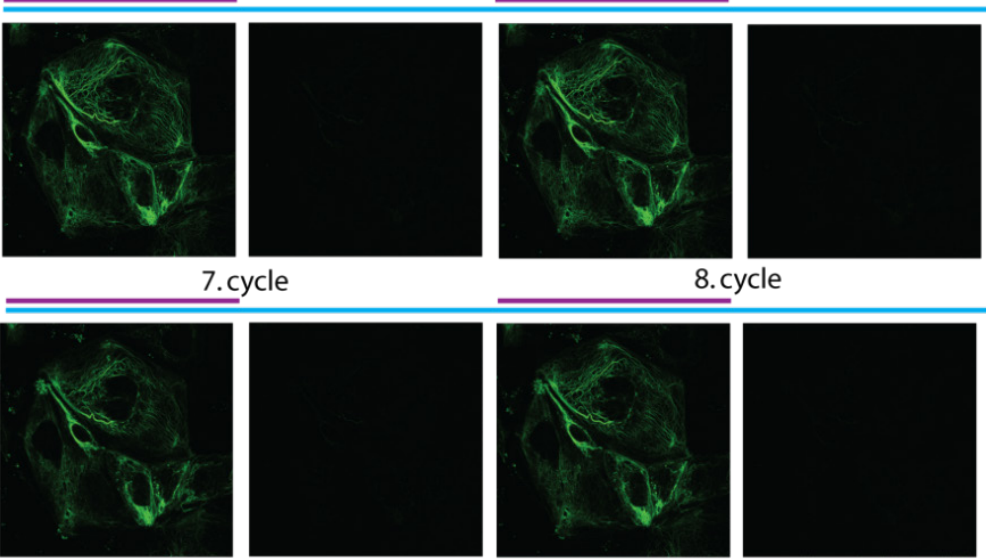

6. cycle
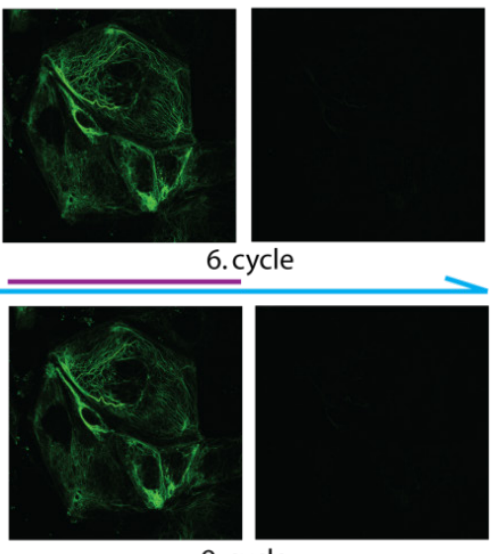

10. cycle
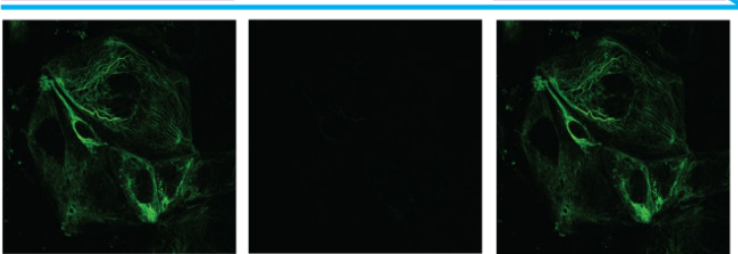

Figure IV.10: Switching of Keratin19-rsEGFP fusion proteins expressed in living PtK2 cells. The proteins were reversibly switched 10 times between the on-state and the off-state. Images of the on-state were recorded by simultaneous irradiation with light of $488 \mathrm{~nm}$ and 405 $\mathrm{nm}$. Off-state images were recorded with light of $488 \mathrm{~nm}$ only. Switching into the on-state was performed by scanning with $488 \mathrm{~nm}$ and $405 \mathrm{~nm}$. Switching into the off-state was performed by scanning with light of $488 \mathrm{~nm}$. Shown are single confocal sections. Scale bar: $25 \mu \mathrm{m}$.

it could be switched several times when expressed in a living cell and fused to a cellular structure (Figure IV.10).

To demonstrate the fast switching speed, low switching fatigue, and high photostability of rsEGFP, it was compared to the well-known and widely used RSFP Dronpa. Both proteins were immobilized in a PAA layer and switched several thousand times by sequential illumination with $491 \mathrm{~nm}$ light of $0.6 \mathrm{~kW} / \mathrm{cm}^{2}$ intensity and $405 \mathrm{~nm}$ light of $2 \mathrm{~kW} / \mathrm{cm}^{2}$ intensity. rsEGFP switched into the onstate with a halftime of $<20 \mu \mathrm{s}$, and, with a halftime of $\sim 1 \mathrm{~ms}$, back into the fluorescent state (Table IV.2). A whole switching cycle took 20 ms (Figure IV.11). In comparison, switching of Dronpa took $250 \mathrm{~ms}$. Hence, in the same time it took Dronpa to perform one single cycle, rsEGFP was switched 12-13 times. 


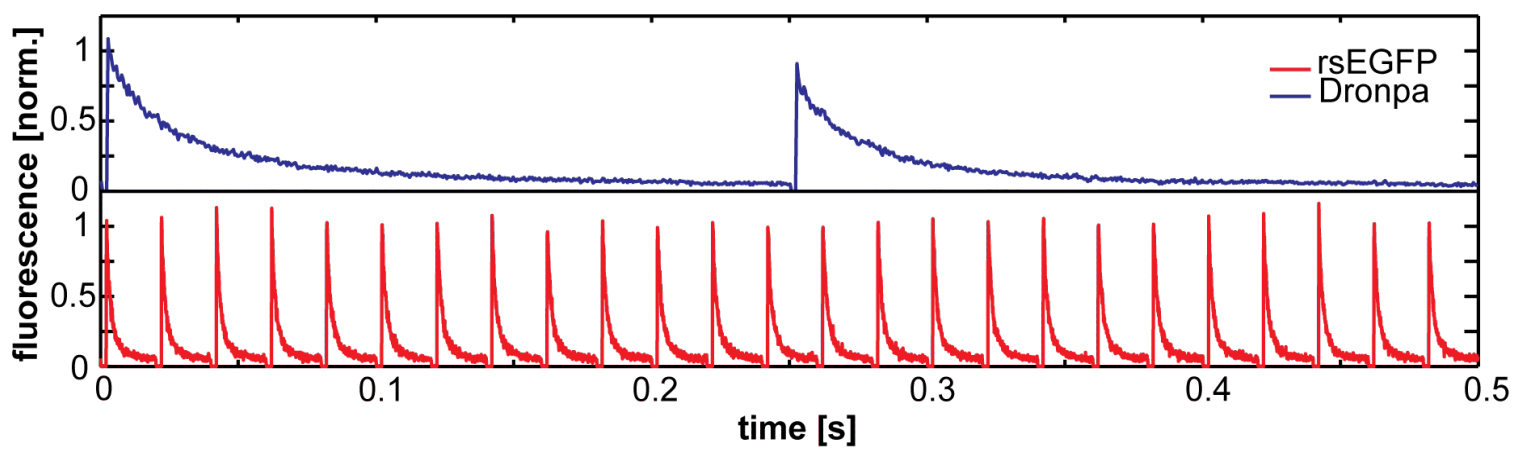

Figure IV.11: Switching curves of Dronpa and rsEGFP. Dronpa (blue) and rsEGFP (red) were immobilized in PAA layer. Switching was performed by alternating irradiation at $405 \mathrm{~nm}$ $\left(2 \mathrm{~kW} / \mathrm{cm}^{2}\right)$ and at $491 \mathrm{~nm}\left(0.6 \mathrm{~kW} / \mathrm{cm}^{2}\right)$. The duration of off-switching at $491 \mathrm{~nm}$ was chosen such that the fluorescence reached a minimum; irradiation with $405 \mathrm{~nm}$ was chosen so that the proteins were fully switched.

Furthermore, Dronpa just allowed $<10$ cycles before it was bleached to half of its initial fluorescence. In comparison the switching fatigue of rsEGFP is strongly reduced. It performed $\sim 1,200$ cycles before being bleached to the same value (Figure IV.12.A)

In addition the conventional bleaching characteristics appearing in the fluorescent state were determined by keeping both proteins in the on-state. This was achieved by continuous irradiation with both $491 \mathrm{~nm}\left(3 \mathrm{~kW} / \mathrm{cm}^{2}\right)$ and $405 \mathrm{~nm}(1$ $\mathrm{kW} / \mathrm{cm}^{2}$ ) light simultaneously. Whereas Dronpa's fluorescence was reduced to $50 \%$ within $\sim 30 \mathrm{~s}$, rsEGFP could be irradiated for $800 \mathrm{~s}$ before it was bleached to the same value (Figure.IV.12.B).
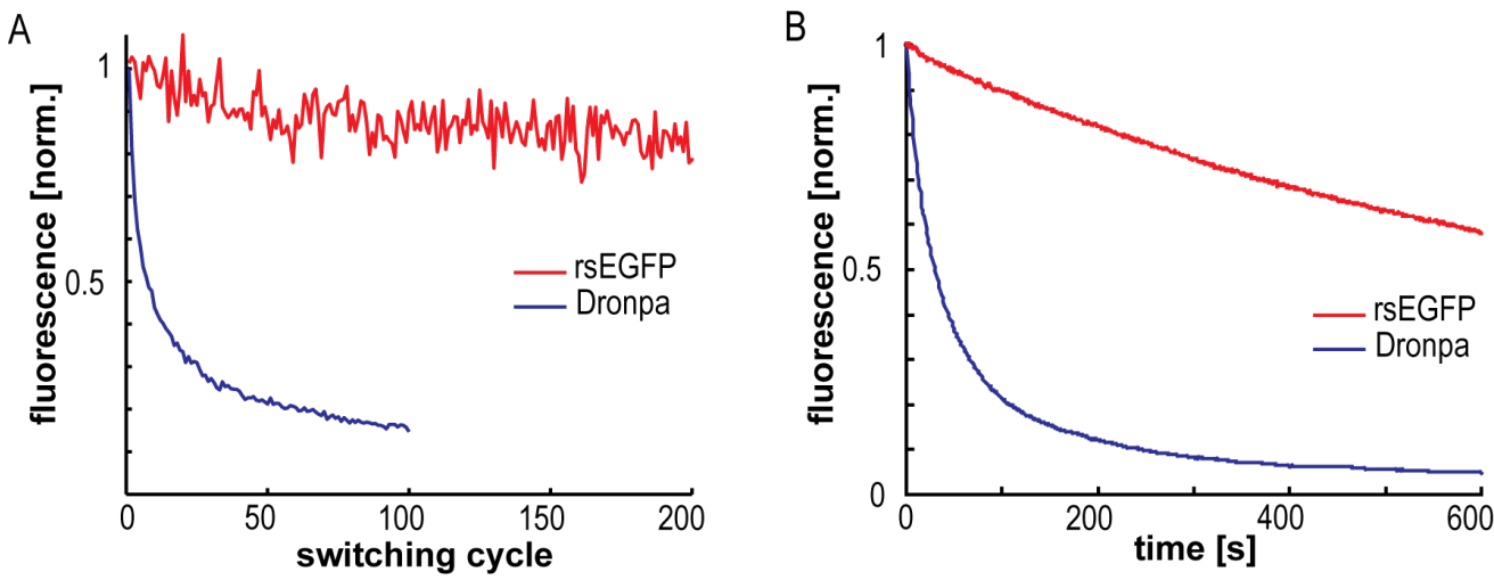

Figure IV.12: Switching fatigue and bleaching characteristics of Dronpa (blue) and rsEGFP (red). (A) The graph shows the number of emitted photons per switching cycle normalized to the initial fluorescence. The same light intensities and switching durations as in Figure IV.13 were used. (B) Shown are the photobleaching characteristics of rsEGFP and Dronpa embedded in a PAA layer. Both proteins were kept in their on-states by continuous irradiation at $405 \mathrm{~nm}\left(1 \mathrm{~kW} / \mathrm{cm}^{2}\right)$, while fluorescence was probed by irradiation at $491 \mathrm{~nm}$ $\left(3 \mathrm{~kW} / \mathrm{cm}^{2}\right)$. 
Hence, compared to Dronpa rsEGFP showed enhancements in all characteristics which are relevant for RESOLFT microscopy. In summary, rsEGFP is a bright, fast switching and photostable RSFP with reduced switching fatigue and a high switching contrast. Beside the advantages for super-resolution imaging these properties are promising for data storage applications.

\section{c. Using rsEGFP as a Rewritable Data Storage Medium}

The potential of RSFPs for high-density all-optical data storage devices has been repeatedly discussed, but the RSFPs known so far were, comparatively, prone to photobleaching and exhibited slow switching speeds. This limited the number of writing and reading cycles and led to slow writing and erasing processes. Hence, data storage with RSFPs was restricted to proof-of-principle demonstrations (Ando, R. et al. 2004; Adam, V. et al. 2010). In contrast, rsEGFP should allow fast writing/erasing, as well as many writing and reading cycles due to its fast switching and reduced switching fatigue, respectively. This was demonstrated with a data storage experiment.

The text of the book "Grimm`s fairy stories" (http://www. gutenberg.org/files/11027/1102 7.txt) including 25 fairy tales was translated into a 7-bit binary ASCII (American Standard Code for Information Interchange) code. These data were sequentially stored on a medium consisting of $a<1 \mu \mathrm{m}$ thin layer of rsEGFP, immobilized in PAA and coated on a
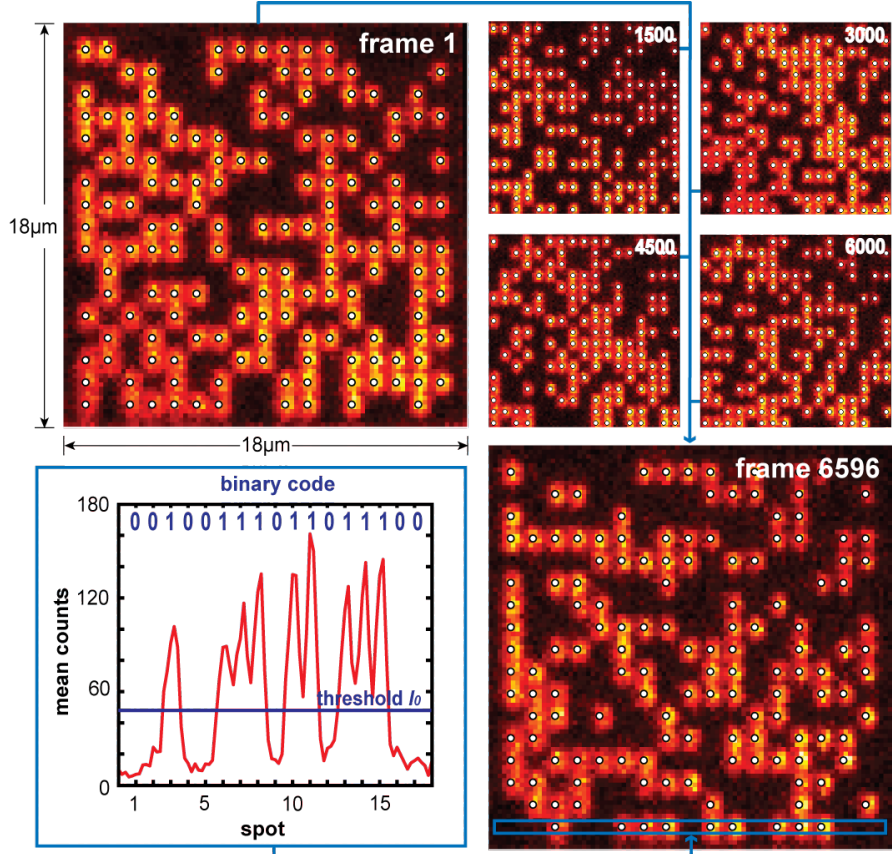

Figure IV.13: Rewritable data storage using rsEGFP. The text of 25 Grimm's fairy stories (ASCll code; $1.9 \mathrm{Mbits}$ ) consecutively written and read on a $17 \times 17 \mu \mathrm{m}$ area of a PAA layer containing rsEGFP, with bits written as spots (representative frames shown). The white dots mark spots that were recognized as ' 1 's. The graph shows an intensity profile along the indicated area, averaged over three pixels along the y-axis. The blue line indicates the threshold $I_{0}$ used to assign read spots to ' 0 's or ' 1 's. 
microscopy slide. A scanning confocal setup (built by Marcel Leutenegger) was used to switch single data spots into an area of 17 $\mu \mathrm{m} \times 17 \mu \mathrm{m}$ of this layer. The spots had a diameter of $\sim 0.5 \mu \mathrm{m}$ and a center-to-center spacing of $1 \mu \mathrm{m}$, corresponding to a storage density similar to the DVD standard $(0.74$ $\mu \mathrm{m}$ pits). Off-switched spots represented ' 0 's and on-switched spots '1's of the binary code. In a first step, all proteins were switched into the off-state by scanning the storage area with $491 \mathrm{~nm}$ light $(0.6$

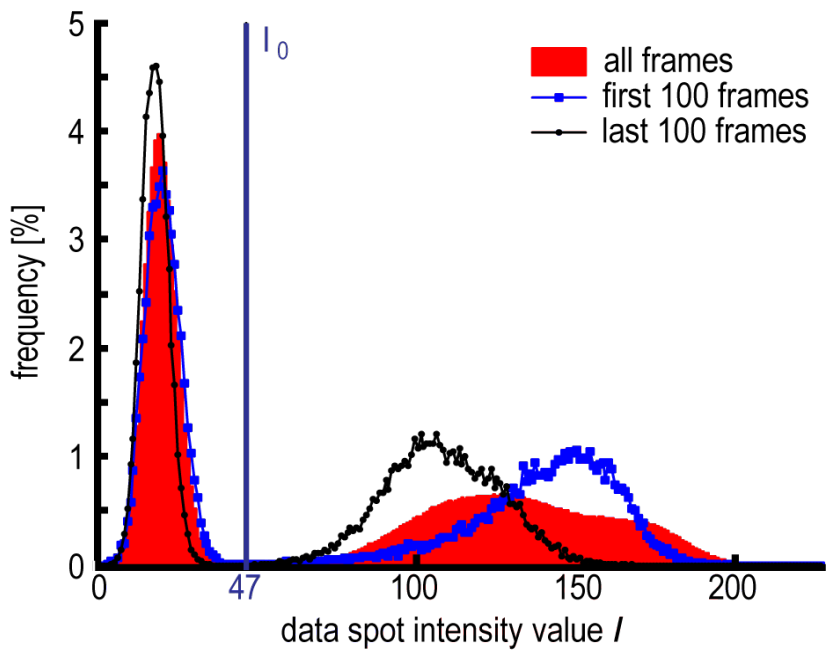

Figure IV.14: Histogram of the data spot fluorescence intensities. Plotted are the detected fluorescence intensities during the optical data storage experiment as shown in Figure IV.13. Shown are the fluorescence intensities of all spots of the first 100 frames (blue squares), the last 100 frames (black points) and the whole experiment (red bars). The shift in the mean counts of the on-state spots represents the photo-bleaching during the recording of the $\sim 6500$ frames.

$\mathrm{kW} / \mathrm{cm}^{2}$; $1.2 \mathrm{~ms}$ per pixel; step size

$500 \mathrm{~nm})$. In a second step, the first 41 characters of the text consisting of 287 bits were stored in this area by switching ' 1 ' spots into the layer using $405 \mathrm{~nm}$ light $\left(\sim 40 \mathrm{~W} / \mathrm{cm}^{2}\right.$ for $10 \mathrm{~ms}$ per bit). Finally, the spot pattern was read out by imaging the storage region with $491 \mathrm{~nm}$ light $\left(0.2 \mathrm{~kW} / \mathrm{cm}^{2} ; 1 \mathrm{~ms}\right.$ dwell time; step size 200 $\mathrm{nm})$. This writing and reading process was repeated 6.596 times on the same storage area until the whole text was sequentially stored and read out again (Figure IV.13). This took $\sim 44$ hours. After storing the whole data set, the fluorescence intensity of on-state spots was reduced by 235 \% (Figure IV.14).

To retrieve the data from the images, the fluorescence intensity was averaged over an area of $0.6 \mu \mathrm{m} \times 0.4 \mu \mathrm{m}(3 \times 2$ pixels $)$ centered on the respective data spot resulting in the intensity value I for each spot. A histogram of all determined I values showed that on- and off-state spots were represented in two different peaks and could be easily separated by determining an intensity threshold $I_{0}$ of 47 (Figure IV.14). With this simple threshold requiring no additional computational effort, the whole text could be read out. The bit error rate was $3.7 \cdot 10^{-6}$ (i.e. 7 bit errors within the whole data set). 
Hence, the properties displayed by rsEGFP constitutes a leap forward in switching speed and endurance which should not just open the door to use fluorescent proteins in data storage applications, but should also make rsEGFP a promising candidate to enable live cell RESOLFT microscopy.

\section{d. Establishing a RESOLFT-type Microscopy Approach Using rsEGFP}

To determine whether rsEGFP enables RESOLFT-type microscopy, a scanning confocal setup was built (constructed by Marcel Leutenegger, Ilaria Testa, and Hannes Bock). It was equipped with three beams: a $405 \mathrm{~nm}$ beam for switching rsEGFP into the fluorescent state, a $491 \mathrm{~nm}$ Gaussian-shaped beam for fluorescence excitation, and a $491 \mathrm{~nm}$ doughnut-shaped beam to switch rsEGFP molecules at the periphery of the excitation area into the off-state. The first imaged samples were living $E$. coli cells, which expressed a fusion protein of rsEGFP (N-terminal) and the prokaryotic actin homologue MreB (C-terminal). The bacteria were immobilized on an agar-coated slide. RESOLFT imaging was performed by repeating an illumination sequence at each scanning position. First, proteins were switched into the on-state by a $100 \mu$ s lasting irradiation step with $405 \mathrm{~nm}$ light of $\sim 1 \mathrm{~kW} / \mathrm{cm}^{2}$ intensity. Afterwards, rsEGFP molecules in the periphery were switched into the off-state by a $10-20$ ms lasting irradiation step with the doughnut-shaped beam of $\sim 1 \mathrm{~kW} / \mathrm{cm}^{2}$ intensity. Finally, the residual onstate proteins in the very center were read out for $1-2$ ms using the $491 \mathrm{~nm}$ Gaussian beam, with an intensity of $\sim 1 \mathrm{~kW} / \mathrm{cm}^{2}$. The light intensities of $\sim 1$ $\mathrm{kW} / \mathrm{cm}^{2}$ were chosen, because non-linear bleaching effects appeared when using substantially higher intensities. To further reduce bleaching, the sample was scanned in a so called "hopping mode". After switching a single spot, the beams were targeted to the next spot, which was further away than the size of the focal spot. Thus, the sample was scanned in several passes by recording only every $N$-th pixel per pass. $N$ was chosen such that rsEGFP could relax from possible long lived excited states, back to the ground state. 

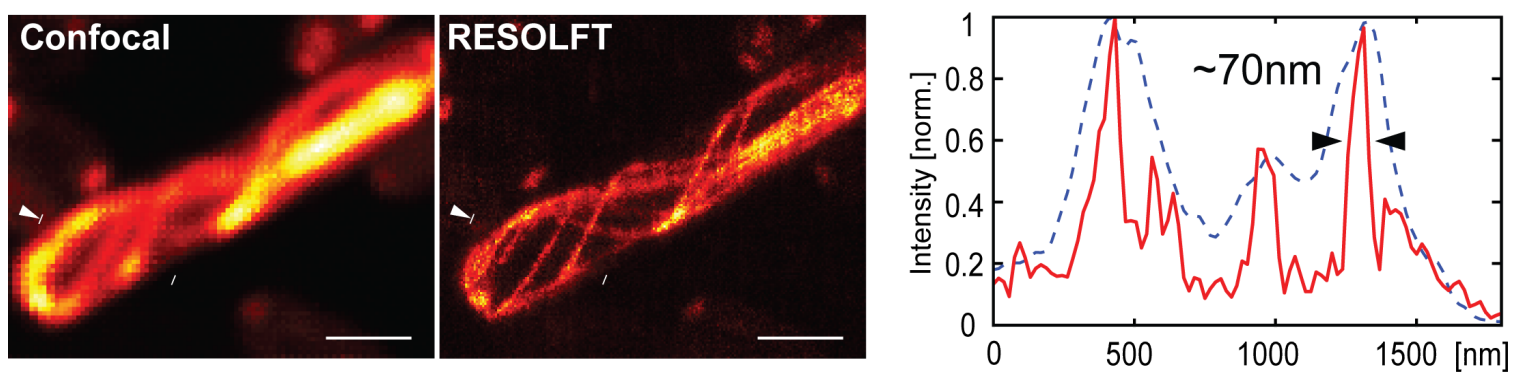

Figure IV.15: RESOLFT microscopy of living E. coli cells expressing rsEGFP-MreB. Shown are the confocal (left) and the corresponding RESOLFT (middle) image. The graph shows the normalized fluorescence profiles between the two white markers with the white arrowhead indicating the direction (solid red, RESOLFT; dashed blue, confocal). Scale bar, $1 \mu \mathrm{m}$

RESOLFT microscopy of an MreB structure in E. coli cells resulted in images of filaments with a full width half maximum (FWHM) of $\sim 70 \mathrm{~nm}$, which was three to four times smaller than the achievable FWHM using conventional diffraction limited confocal laser scanning microscopy (Figure IV.15). This is why the bacterial cytoskeleton is more clearly revealed by RESOLFT microscopy than by its confocal counterpart. Furthermore, filaments, which lay in close proximity, could be separated by RESOLFT, whereas such filaments appeared as a single
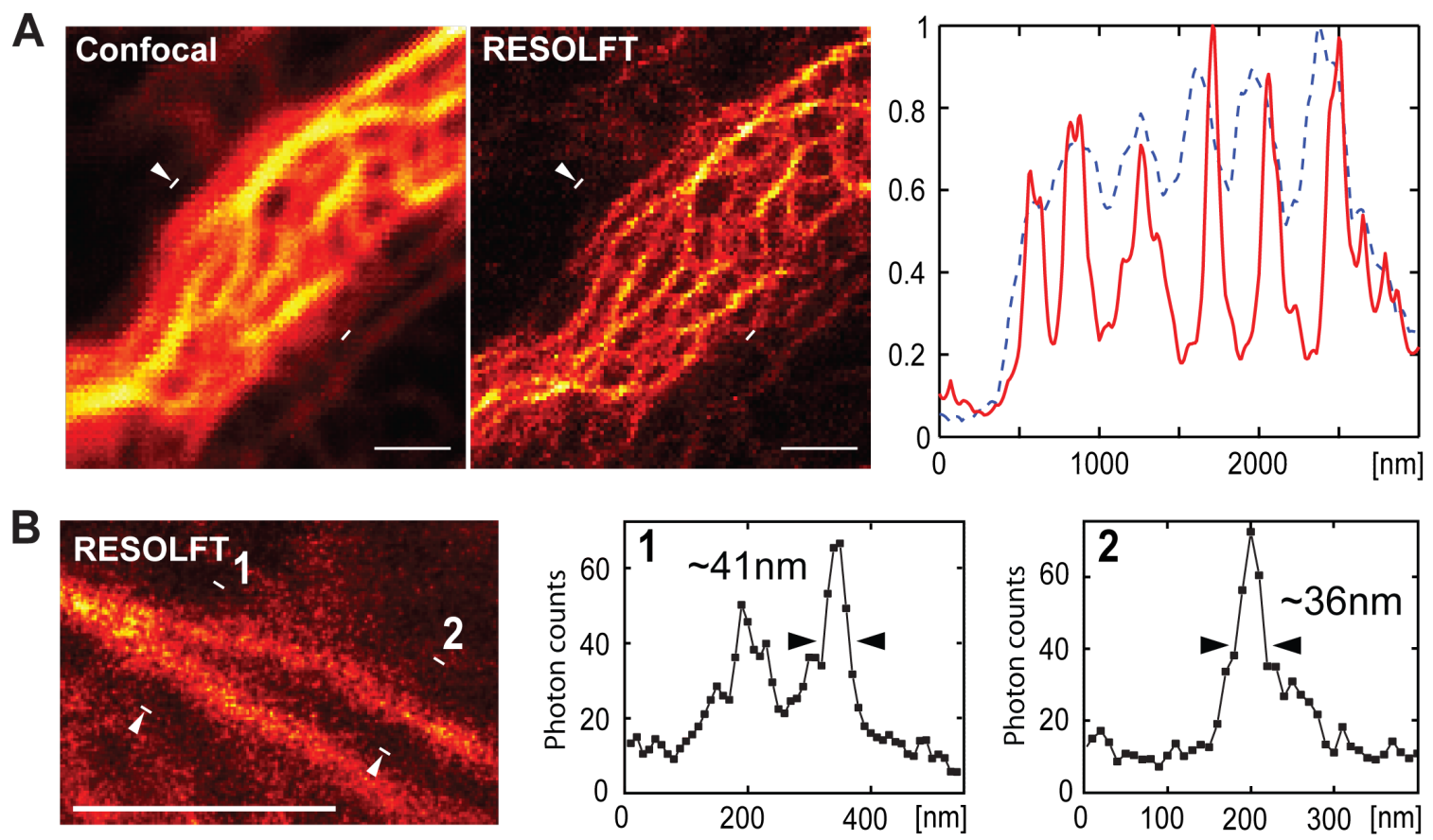

Figure IV.16: RESOLFT microscopy of living PtK2 cells expressing Keratin19-rsEGFP. (A) Mammalian (PtK2) cell expressing Keratin-19-rsEGFP imaged in the confocal (left) and the RESOLFT (middle) mode. The graph shows the normalized fluorescence profiles between the two white markers with the white arrowhead indicating the direction (solid red, RESOLFT; dashed blue, confocal). (B) RESOLFT image (left) of keratin19-rsEGFP filaments in a PtK2 cell recorded with a pixel size of $10 \mathrm{~nm} \times 10 \mathrm{~nm}$; smoothed with a low-pass Gaussian filter of 1.2 pixel width. Graphs 1 and 2 extracted from the image as indicated reveal a resolution $<40 \mathrm{~nm}$. Scale bar, 1 $\mu \mathrm{m}$. 
blur in the diffraction limited confocal image. Up to ten images of the same structure could be recorded before the sample was bleached.

In a next step, RESOLFT microscopy was used to image structures of living mammalian cells. To this end, rsEGFP was fused to Keratin19 and expressed in PtK2 cells. Again the keratin filament was more precisely revealed by RESOLFT imaging compared to CLSM (Figure IV.16.A). The keratin filament could be imaged several times before getting bleached. To determine the achievable resolution of rsEGFP-based RESOLFT microscopy, thin keratin filaments were imaged with a pixel step size of $10 \mathrm{~nm}$. Line profiles of these filaments indicated a resolution $<40 \mathrm{~nm}$, corresponding to a 5-6 fold improvement compared to confocal microscopy (Figure IV.16.B).
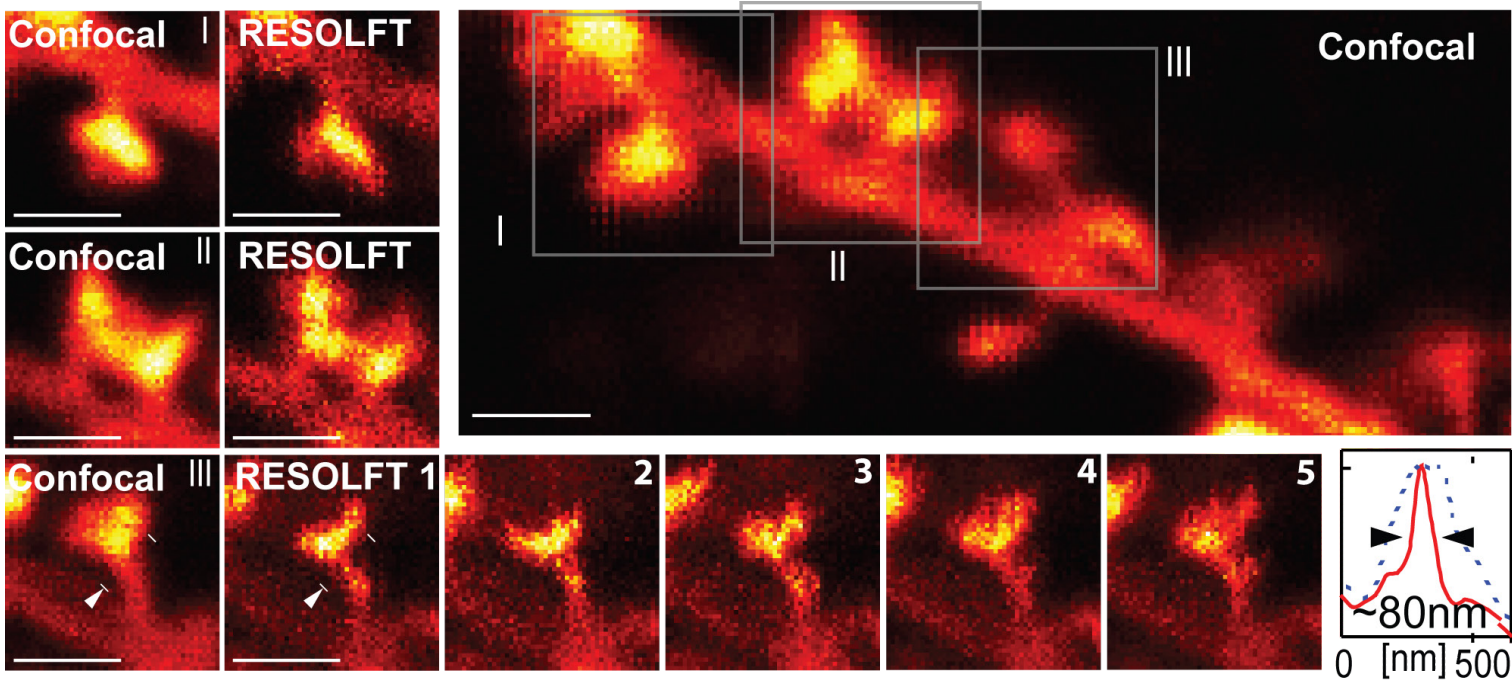

Figure IV.17: RESOLFT microscopy of living organotypic hippocampal mice slice expressing Lifeact-rsEGFP. Main image: confocal overview. I-III: three spines, as indicated on the main image, each imaged in the confocal (left) and the RESOLFT mode (right). Spine III was repeatedly imaged in the RESOLFT mode within $5 \mathrm{~min}$, demonstrating the changes over time. Graph: normalized profile across a spine neck as imaged in the RESOLFT (solid red) or the confocal mode (dashed blue) between the two white markers. Scale bars, $1 \mu \mathrm{m}$.

Finally, the RESOLFT microscopy approach was transferred to living brain tissue imaging. Viral particles carrying a Lifeact-rsEGFP construct were locally injected into cultured organotypical hippocampal mice brain slices (performed by Nicolai Urban). Expressed in neurone cells, the 17 amino acid long Lifeact peptide binds to actin (Riedl, J. et al. 2008). Single spines of a dendrite were imaged. RESOLFT revealed fine morphological differences between the spines (Figure IV.17). Line profiles across a spine neck showed a FWHM of $\sim 80 \mathrm{~nm}$, suggesting a resolution $\leq 80 \mathrm{~nm}$. Furthermore, a single spine was imaged repeatedly over 5 
minutes. During that time morphological changes of the spine shape were detected.

To investigate, if the resolution enhancement using rsEGFPRESOLFT can be transferred to the third dimension, a fourth beam path of $491 \mathrm{~nm}$ light was added to the microscopic setup. It was equipped with a z-phase-plate (Harke, B. et al. 2008). The resulting PSF showed additional intensity maxima above and below the focal plane, so that fluorophores above and below this plane were switched off. This should lead to an effectively
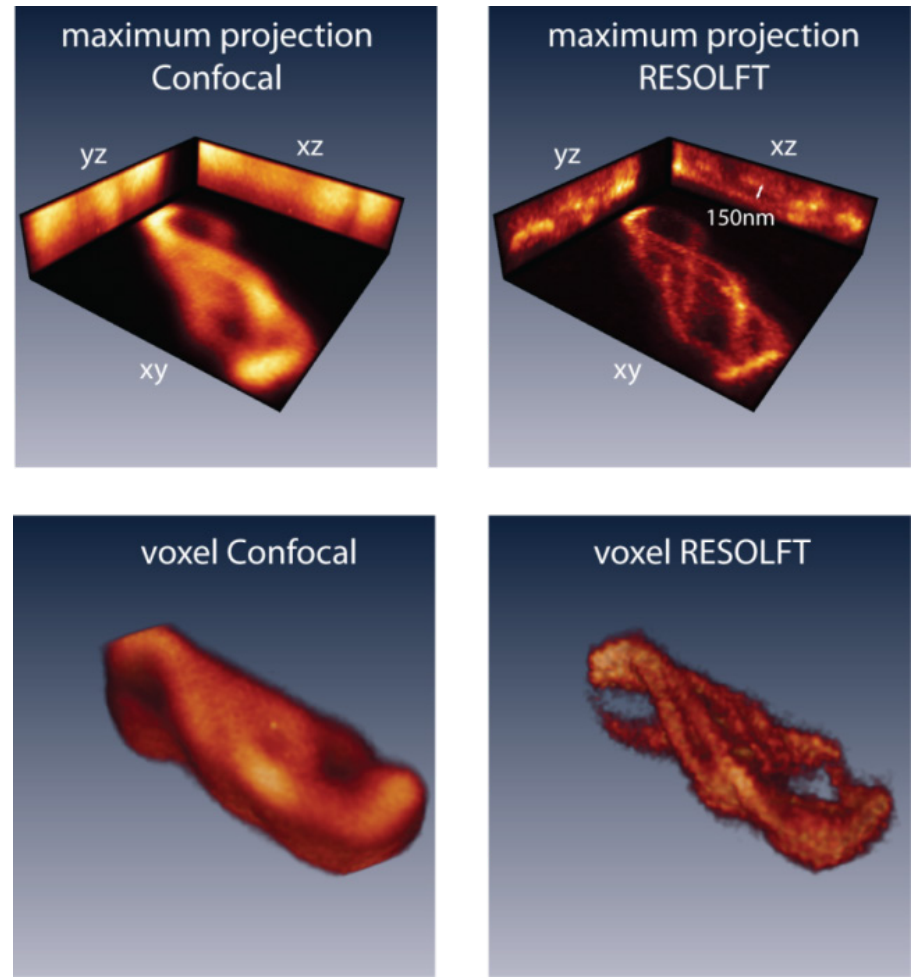

Figure IV.18: 3D RESOLFT imaging of living $E$. coli cells expressing rsEGFP-MreB. A z-phase-plate was used to decrease the effective PSF also in the axial dimension. The pixel size was $35 \mathrm{~nm} \times 35 \mathrm{~nm} \times 50 \mathrm{~nm}$. Shown are the maximum projections (top) of confocal and RESOLFT image stacks as well as $3 \mathrm{D}$ voxel reconstructions (bottom).

smaller PSF in the axial

dimension. A complete image stack of $E$. coli cells expressing rsEGFP-MreB fusion proteins was recorded. The pixels size was $35 \mathrm{~nm} \times 35 \mathrm{~nm} \times 50 \mathrm{~nm}(\mathrm{x} \times \mathrm{y}$ $\times z)$. The maximal projection and 3D voxel reconstruction of the image stack demonstrated an enhanced resolution in all three dimensions (Figure IV.18).

Hence, the enhanced properties of rsEGFP enabled live cell RESOLFT microscopy for the first time. The diffraction barrier was substantially overcome by applying light of just $\sim 1 \mathrm{~kW} / \mathrm{cm}^{2} ; \sim 1,000,000$-fold less intensity compared to STED microscopy. The reduced switching fatigue of rsEGFP allowed several images of the same structure to be recorded, enabling time-lapse imaging on the nanoscale. 


\section{e. Establishing a Diffraction-Unlimited all Optical Data}

\section{Storage Approach}

In the next step, the data storage and the RESOLFT application ought to be fused to a single application: RESOLFT optical data storage. This method should allow storing data by writing and reading smaller data spots in a shorter distance than regular focusing would allow.

To investigate subdiffraction-resolution writing, an rsEGFP-PAA layer was prepared as already used in Chapter IV.1.c. To create data spots of diffractionunlimited sizes in this layer, a three step approach was established. First, proteins at a defined position were switched into the fluorescent state by applying $405 \mathrm{~nm}$ light with an intensity of $1 \mathrm{~kW} / \mathrm{cm}^{2}$ for $100 \mu \mathrm{s}$ (Figure IV.19). After a $2 \mathrm{~ms}$ equilibration time the $491 \mathrm{~nm}$ doughnut-shaped beam with an intensity of 0.5 $\mathrm{kW} / \mathrm{cm}^{2}$ illuminated the spot for $20 \mathrm{~ms}$. This decreased the area of proteins in the on-state, by switching those in the periphery, off. The result is a small residual spot of proteins in the on-state in the center. Subsequently, these on-state proteins in the center of the spot were transferred into a permanent non fluorescent state by bleaching with intense $532 \mathrm{~nm}$ light $\left(\sim 900 \mathrm{~kW} / \mathrm{cm}^{2}\right)$. This was possible since light of this wavelength and intensity bleaches rsEGFP proteins in the on-state more effectively than those in the off-state (Figure IV.20). It has to be noted that $532 \mathrm{~nm}$ light bleaches on-state proteins, but also, in a competing

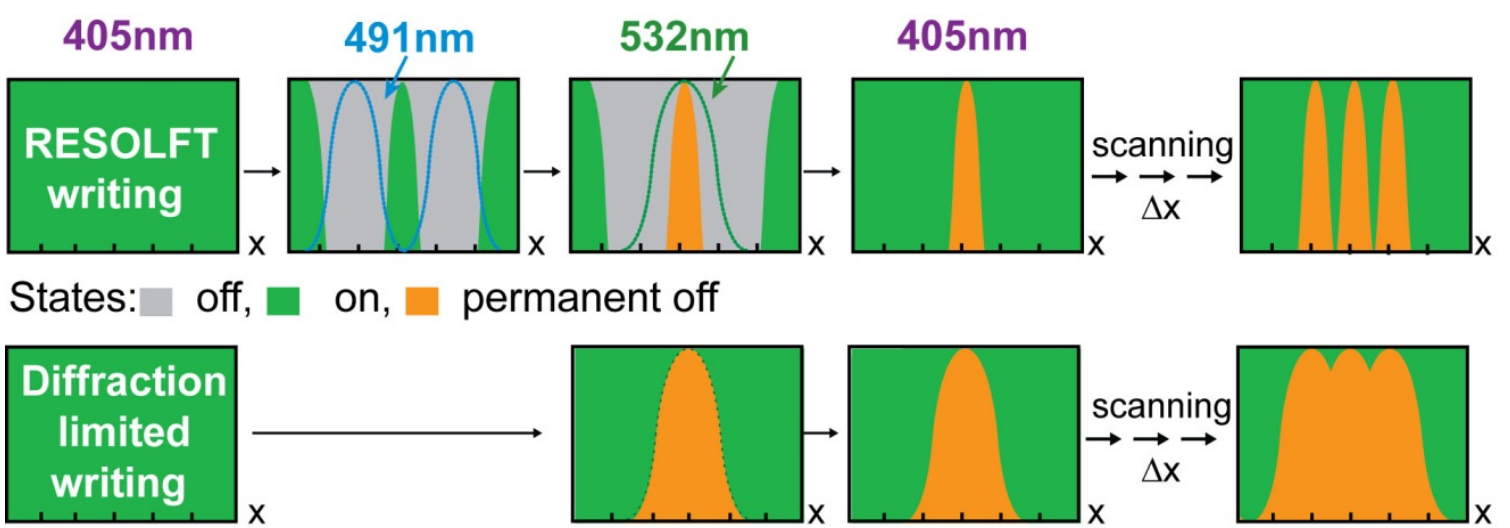

Figure IV.19: Schematic of RESOLFT-type writing (top) versus diffraction limited writing (bottom). In RESOLFT-type writing rsEGFP molecules are switched off at $491 \mathrm{~nm}$ using a doughnut-shaped focal intensity (dashed blue line) so that the on-state is confined to a subdiffraction-sized region around the doughnut center. Subsequently, irradiation with light of $532-\mathrm{nm}$ transfers on-state molecules into a permanent bleached state. Irradiation at $405 \mathrm{~nm}$ switches the off-state molecules back into the on-state, allowing the writing of another feature in subdiffraction proximity. 
process, switches these proteins off. Therefore, to achieve the highest possible contrast between on-state and off-state bleaching $(\sim 3.5: 1)$ the switching sequence of on-switching (405 nm), off-switching in the periphery (491 $\mathrm{nm}$; doughnut shaped beam) and bleaching (532 $\mathrm{nm}$ ) had to be repeated 50 times (Figure IV.20). This resulted in a final non-fluorescent data spot.

Based on this writing scheme, nine different spot patterns of $3 \times 3$ bit fields with separations of $250 \mathrm{~nm}$ were written into the rsEGFP layer and subsequently read-out with RESOLFT microscopy. While writing and reading with conventional focusing blurred the data, the single bits were fully discernible

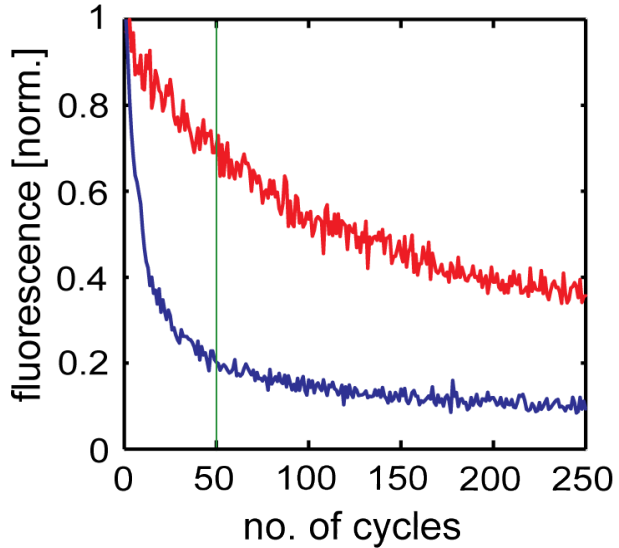

Figure IV.20: On-state specific bleaching of rsEGFP. Shown is a comparison of the on-state bleaching (blue curve) with the off-state bleaching (red curve). The total exposure times and applied intensities were the same in both cases, only the sequence of irradiation was different. Plotted are the respective fluorescence intensities recorded at the beginning of irradiation with $491 \mathrm{~nm}$ against the number of the switching cycle.

when using the RESOLFT optical data storage mode (Figure IV.21.A). This RESOLFT-type approach allowed for the writing and reading of discernible spots with separations as low as $200 \mathrm{~nm}$ (Figure IV.21.B).

Hence, using rsEGFP in combination with a RESOLFT-type writing approach allowed to write spots of smaller sizes than it is possible with conventional optical

A

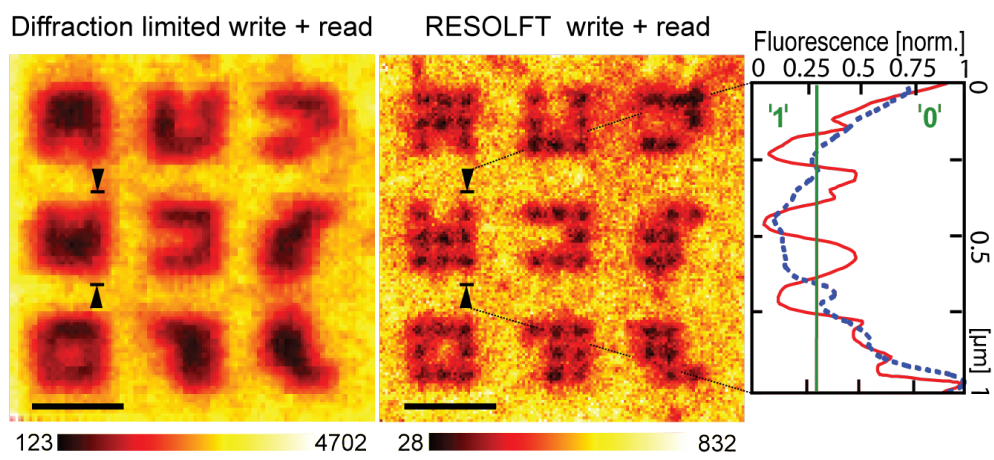

B

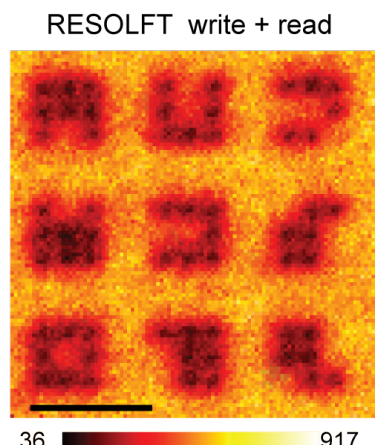

Figure IV.21: Subdiffractional writing using rsEGFP and visible light. (A) Conventional (left) and subdiffraction RESOLFT (middle) joined writing and reading in a layer of immobilized rsEGFP. The outlines of the corresponding 333 bit patterns were identical. The distance between two bleached spots was $250 \mathrm{~nm}$ in each case. Right, normalized line profiles of the fluorescence signal between the two arrows (solid red, RESOLFT; dashed blue, confocal). (B) RESOLFT writing and reading with bit distances of $200 \mathrm{~nm}$. The displayed image represents an average of four recordings. Scale bars: $1 \mu \mathrm{m}$. 
focusing. Furthermore, this approach overcame the limitation in optical data storage density for the first time.

\section{Reversibly Switchable Enhanced Green}

\section{Fluorescent Protein 2 (rsEGFP2)}

The generation of rsEGFP enabled RESOLFT measurements of living cells and tissues for the first time. The enabling element was the enhanced switching characteristics of rsEGFP, as compared to other RSFPs; most notably, reduced switching fatigue and increased switching speed. rsEGFPs switching kinetics facilitated dwell times per pixel of $\sim 20 \mathrm{~ms}$ in RESOLFT imaging. Depending on the scanning step size (normally $20 \mathrm{~nm}$ to $40 \mathrm{~nm}$ depending on the imaged structure and the required resolution), the imaging speed was $12.5-50 \mathrm{~s} / \mu^{2}$. This was sufficient to image cytoskeleton structures of living cells. However, the imaging of faster moving structures, like free diffusing molecules, was still challenging due to the fact that a fast movement of rsEGFP molecules into and out of the focal spot results in a destruction of the switching pattern. Hence, to enable RESOLFT imaging of faster moving structures, the switching speed of rsEGFP had to be further enhanced. This was achieved by the generation of rsEGFP2.

\section{a. Generation of rsEGFP2}

During the generation process of rsEGFP, a mutation in the first of the three chromophore forming amino acids, which was sufficient to make mEGFP switchable, was found. While establishing rsEGFP, care had been taken to retain the key amino acids of EGFP, including the chromophore amino acids, to ensure the preservation of its enhanced characteristics such as brightness, maturation time, and photostability. Hence, the T65A mutation was not utilized for the generation of rsEGFP. However, mEGFP(T65A) showed fast negative switching properties and low switching fatigue, which made this protein an interesting base for the generation of a faster switching rsEGFP variant for RESOLFT microscopy. 
The residual fluorescence in the off-state of mEGFP(T65A) is $50 \%$, which is too high for RESOLFT applications. Further optimizations were achieved by combining T65A with the rsEGFP mutations. Introducing the initial switching mutation of rsEGFP Q69L into mEGFP(T65A) already resulted in a fast switching variant with reduced residual fluorescence in the off-state of $\sim 7 \%$. The additional introduction of the mutation V163S (known also from rsEGFP) led to EGFP(T65A,Q69L,V163S,A206K) (Figure IV.22). It still showed fast switching properties and minimal switching fatigue. The residual fluorescence in the offstate was further reduced to $3.5 \%$. These characteristics should make this variant suitable for RESOLFT applications. It was named rsEGFP2.

Random mutations of the positions V150 and S205, which were relevant mutation sites in the generation process of rsEGFP, did not show any improvement in RESOLFT relevant characteristics, instead they reduced the switching contrast and/or switching speeds, and were not considered further.

Figure IV.22: Alignment of the amino acid sequences of EGFP, rsEGFP, and rsEGFP2. Differences are highlighted. 


\section{b. Characterization of rsEGFP2}

At equilibrium, rsEGFP2 adopts the fluorescent state. rsEGFP2's extinction coefficient of $\varepsilon=61.000$ was slightly higher, and the fluorescence quantum yield of $\Phi_{F I}=0.3$ slightly lower than those of rsEGFP (Table. IV.3). Hence, both proteins exhibited the same brightness. In the on-state, rsEGFP2 exhibited a

Table IV.3: Properties of EGFP, rsEGFP and rsEGFP2 (*(Patterson, G.H. et al. 1997))

\begin{tabular}{|c|c|c|c|c|c|c|}
\hline & $\begin{array}{c}\text { extinction } \\
\text { coefficient } \\
(\varepsilon) \\
{\left[\mathrm{M}^{-1} \mathrm{~cm}^{-1}\right]}\end{array}$ & $\begin{array}{l}\text { fluorescence } \\
\text { quantum } \\
\text { yield } \\
\left(\Phi_{\mathrm{FL}}\right)\end{array}$ & $\begin{array}{c}\text { excitation } \\
\text { maximum } \\
\text { on-state } \\
\text { [nm] }\end{array}$ & $\begin{array}{c}\text { single } \\
\text { molecule } \\
\text { brightness } \\
\text { [norm.] }\end{array}$ & $\begin{array}{c}\text { emission } \\
\text { maximum } \\
{[\mathrm{nm}]}\end{array}$ & $\begin{array}{c}\text { absorption } \\
\text { maximum } \\
\text { off-state } \\
{[\mathrm{nm}]}\end{array}$ \\
\hline EGFP & $53.000^{*}$ & $0.6^{*}$ & 489 & 1 & 509 & n.a. \\
\hline rsEGFP & 47.000 & 0.36 & 493 & 0.6 & 510 & 396 \\
\hline rsEGFP2 & 61.300 & 0.3 & 478 & 0.6 & 503 & 408 \\
\hline
\end{tabular}

n.a.: not applicable

single absorption peak at $\sim 480 \mathrm{~nm}$ (Figure IV.23.A). Excitation into this band led to fluorescence at $503 \mathrm{~nm}$. Hence, the excitation and fluorescence spectra of rsEGFP2 are blue shifted in comparison to rsEGFP. rsEGFP2 exhibits a negative switching mode. In a competing process, the fluorescence excitation light also switched the protein into the non-fluorescent state, accompanied by a change in the absorption spectrum (Figure IV.23.B). The off-state exhibits a single absorption band peaking at $408 \mathrm{~nm}$. Hence the off-state absorption peak was red shifted in comparison to the one of rsEGFP. Ensemble measurements of rsEGFP2 revealed a residual fluorescence in the off-state of $\sim 3.5 \%$ compared to

A

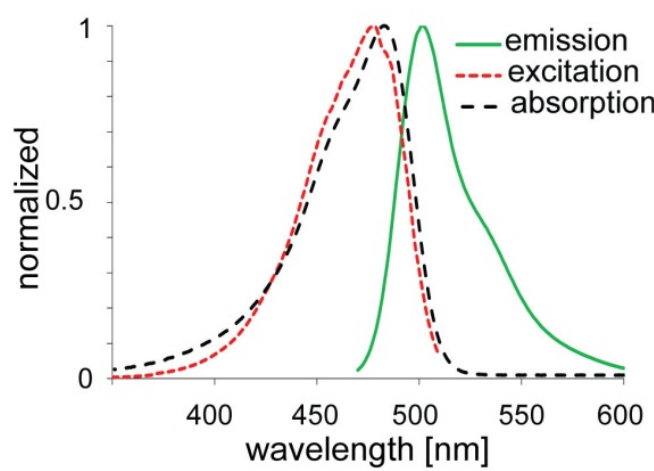

B

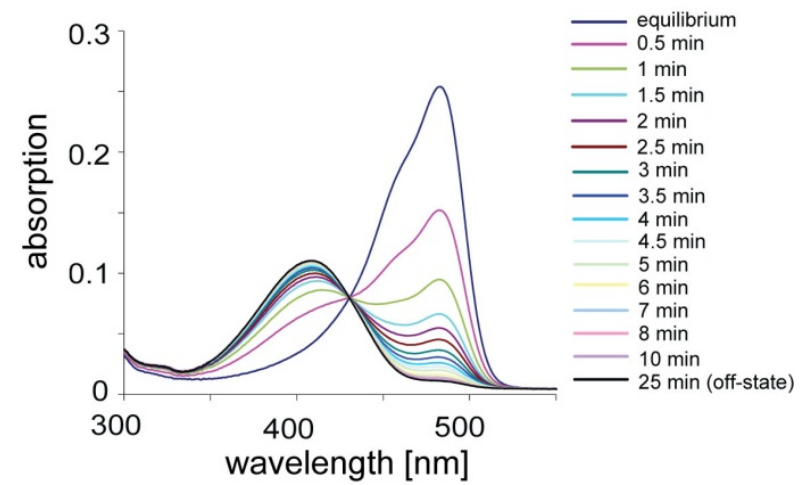

Figure IV.23: Spectral properties of rsEGFP2. (A) Absorption, excitation, and emission spectra of rsEGFP2 in the fluorscent equilibrium state. Spectra were determined using purified protein at $\mathrm{pH}$ 7.5. (B) Changes in the absorption spectrum of rsEGFP2 during switching from the equilibrium to the off-state. The spectra were taken after different times of illuminating a cuvette of rsEGFP2 protein solution with $488 \mathrm{~nm}$ light. 


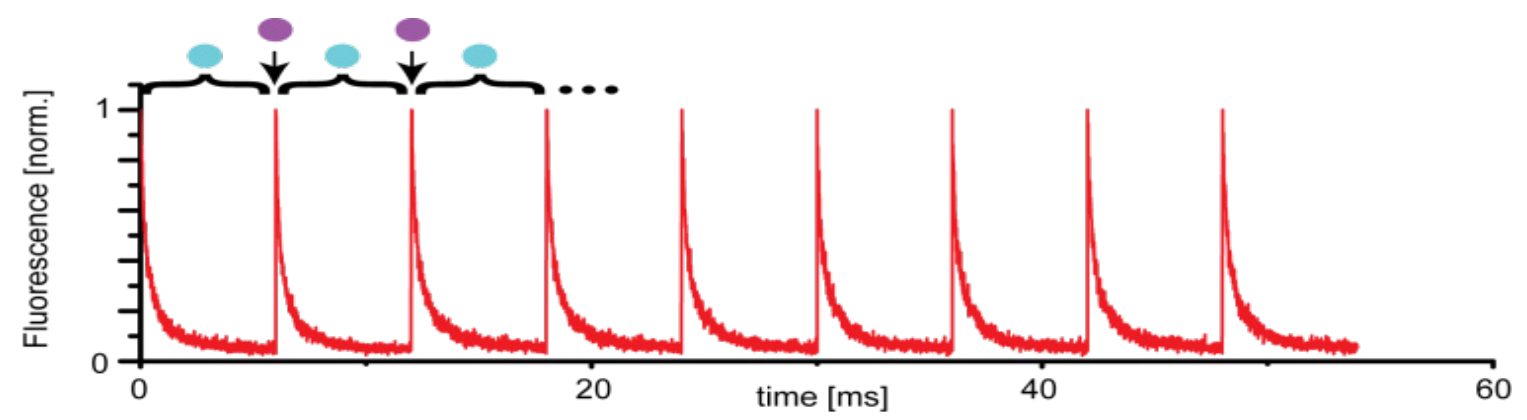

Figure IV.24: Switching curves of rsEGFP2. Switching was performed on immobilized rsEGFP2 porotein in a PAA layer by alternating irradiation with $491 \mathrm{~nm}\left(\sim 7 \mathrm{~kW} / \mathrm{cm}^{2}, 6 \mathrm{~ms}\right.$, blue marked area) and $405 \mathrm{~nm}$ light $\left(\sim 3 \mathrm{~kW} / \mathrm{cm}^{2}, 100 \mu \mathrm{s}\right.$, purple marked area).

the on-state fluorescence. The illumination of proteins in the off-state with light of $\sim 405 \mathrm{~nm}$ switched the protein back into the fluorescent state, hence switching is reversible (Figure IV.24). The determination of the absorption spectra of rsEGFP2 at different $\mathrm{pH}$ values showed a single $480 \mathrm{~nm}$ peak at alkaline and neutral conditions. By decreasing the $\mathrm{pH}$ value, this on-state peak disappeared and the off-state peak at $408 \mathrm{~nm}$ appeared (Figure IV.25). This demonstrates that the chromophore in the on-state is deprotonated, whereas it is protonated in the off-state, as already shown for rsEGFP. The $p K a$ is $\sim 5.8$.

To determine, whether rsEGFP2 can be used as a fusion marker in live cell approaches its oligomerization state was analyzed. Size separation experiments by semi-native gel electrophoresis revealed, that rsEGFP2 acts as a true monomer at room temperature (Figure IV.26). Due to the fact that folding and maturation are crucial characteristics of an FP in the context of live cell studies, the maturation time of rsEGFP2 was determined by purifying nonmatured protein and detecting the fluorescence increase of the protein solution at $37^{\circ} \mathrm{C}$ over time (Figure IV.27). The rsEGFP2 chromophore

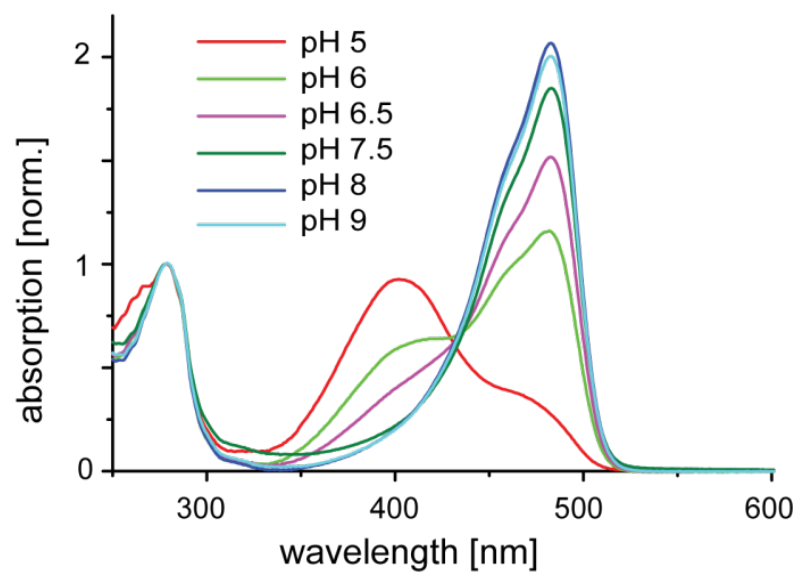

Figure IV.25: Absorption spectra of equilibrium-state rsEGFP2 at different $\mathrm{pH}$ values. Purified rsEGFP2 protein was diluted in various buffers (pH5: $0.1 \mathrm{M}$ citrate buffer, $\mathrm{pH} \mathrm{6,7:}$ $0.1 \mathrm{M}$ Tris- $\mathrm{HCl}$ buffer, $\mathrm{pH}$ 9, 10: $0.1 \mathrm{M}$ glycine buffer). 
showed fast maturation with a halftime of $\sim 20$ min. Hence, rsEGFP2 was checked for its usability as a fusion marker by generating a variety of different fusion proteins and expressing them for 24 - 48 hours in PtK2 cells. Afterwards, the cells were imaged using CLSM (Figure IV.28). All

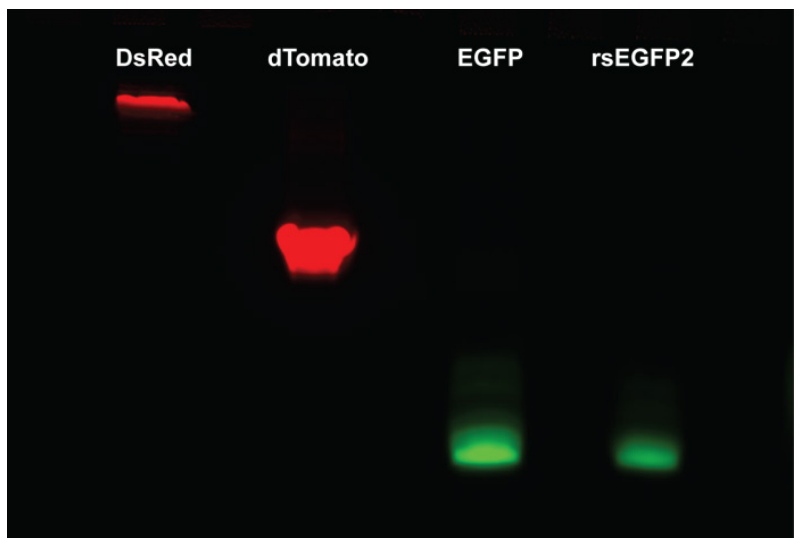
fusion proteins were expressed and

Figure IV.26: rsEGFP2 is a monomer. Seminative polyacrylamide gel electrophoresis of exhibited. Morphologies typically rsEGFP2. Purified monomeric EGFP, dimeric dTomato, tetrameric DsRed, and rsEGFP were expected of respective expressions separated on a semi-native gel. Images were taken with a custom-built gel documentation system. To detect green fluorescence (EGFP and rsEGFP) the gel was irradiated with light of $470 \pm$ $5 \mathrm{~nm}$ and fluorescence was detected at $525 \pm 30$ $\mathrm{nm}$. To detect red fluorescence (dTomato and DsRed) the gel was irradiated with light of $545 \pm$

To investigate whether rsEGFP2 $10 \mathrm{~nm}$ and fluorescence was detected at $617 \pm 37$. exhibits faster switching speeds in Both images were overlaid and are represented in comparison to rsEGFP, the off-

switching kinetics of both proteins were determined, since off-switching is the most time consuming step in RSFP-RESOLFT. To this end, both proteins were fused to Keratin and expressed in PtK2 cells. At a single position, switching cycles were performed for both proteins in the living cell. This was done by alternated irradiation with light of $405 \mathrm{~nm}$ and $491 \mathrm{~nm}$ wavelengths. Care was taken that in each illumination step proteins were completely switched on/off. The off-switching kinetics were determined for different $491 \mathrm{~nm}$ light intensities (Figure IV.29.A). rsEGFP2 switched up to $\sim 6.5$ times faster than rsEGFP. Interestingly, the difference in speeds between the proteins became less under higher intensities. This indicated non-linear effects of the light intensity on the switching speed

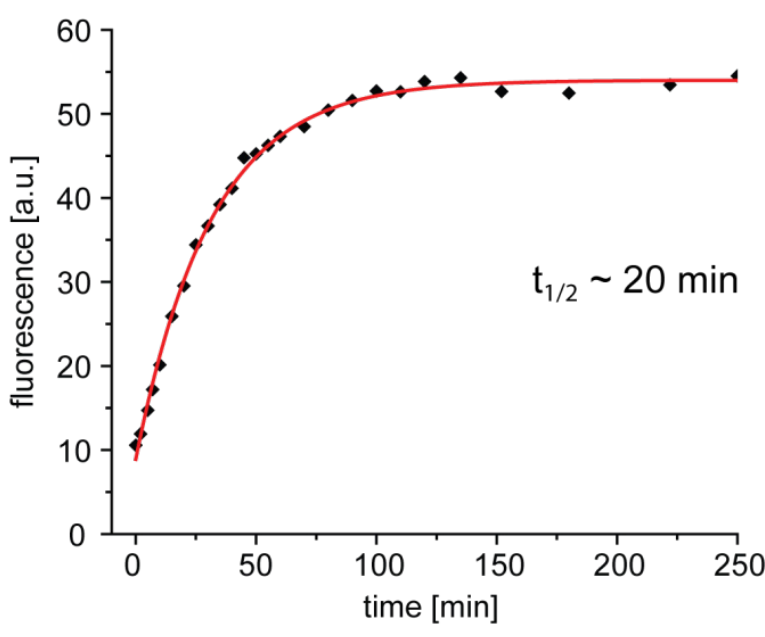

Figure IV.27: Maturation kinetic of rsEGFP2 at $37^{\circ} \mathrm{C}$ 

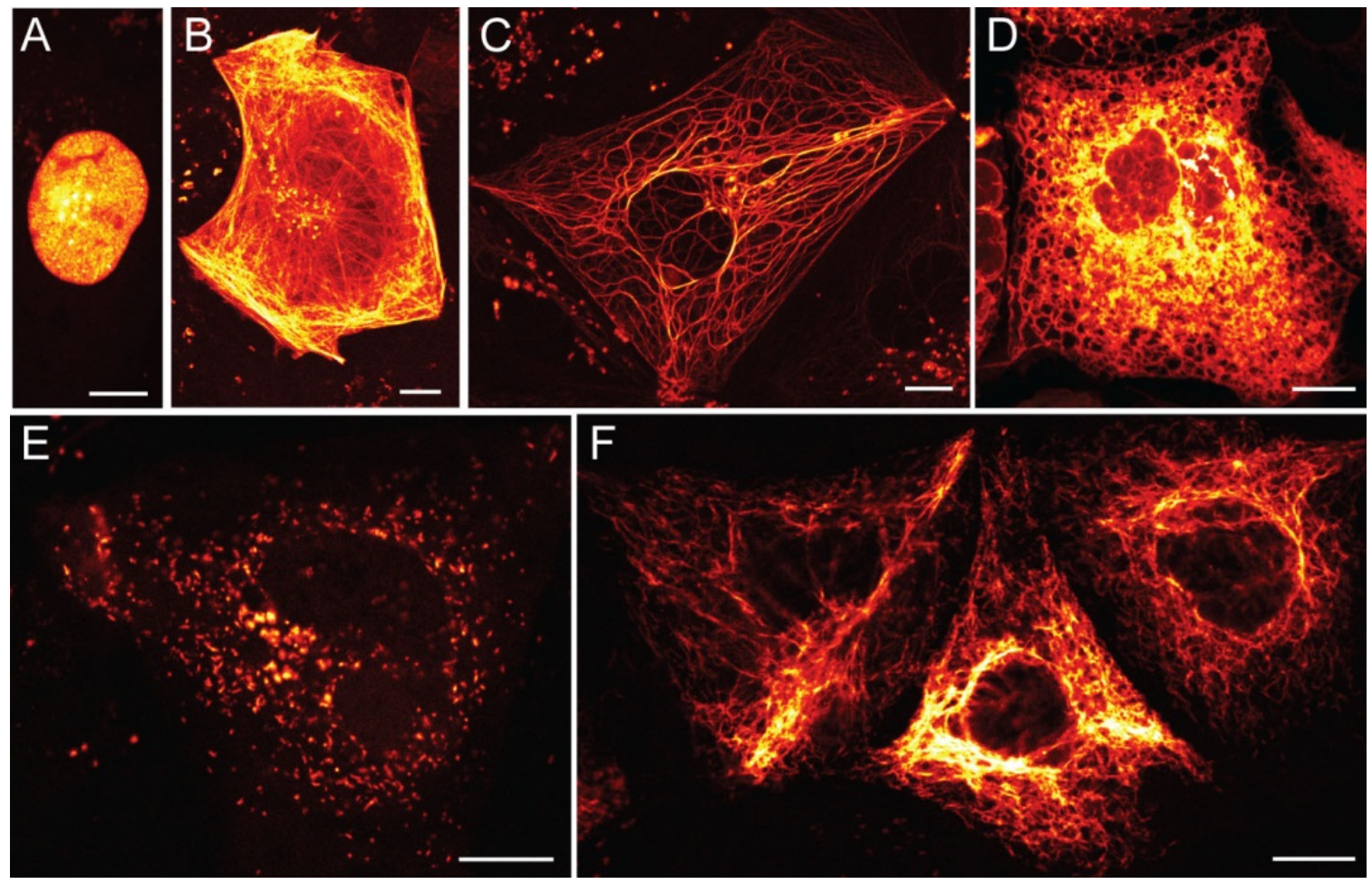

Figure IV.28: Expression of various functional rsEGFP2 fusion proteins in mammalian PtK2 cells. (A) Histone H2B-rsEGFP2, (B) rsEGFP2-MAP2, (C) Keratin19-rsEGFP2, (D) rsEGFP2ER(retention signal), (E) Pex16-rsEGFP2, and (F) Vimentin-rsEGFP2. Shown are single confocal sections (A) and maximum projections (B-F) of living cells. Fluorescence was excited by simultaneous irradiation with light of $488 \mathrm{~nm}$ and $405 \mathrm{~nm}$. Scale bars: $10 \mu \mathrm{m}$.

(Figure IV.29.B). To investigate the switching fatigue of rsEGFP2, 4000 cycles were performed for both proteins immobilized in a PAA layer by alternate irradiation with $405 \mathrm{~nm}\left(3 \mathrm{~kW} / \mathrm{cm}^{2}\right)$ and $491 \mathrm{~nm}\left(7 \mathrm{~kW} / \mathrm{cm}^{2}\right)$ light. Illumination times were chosen such that the fluorescence signal reached a maximum/minimum. The experimental results revealed a $\sim 3$-fold reduced
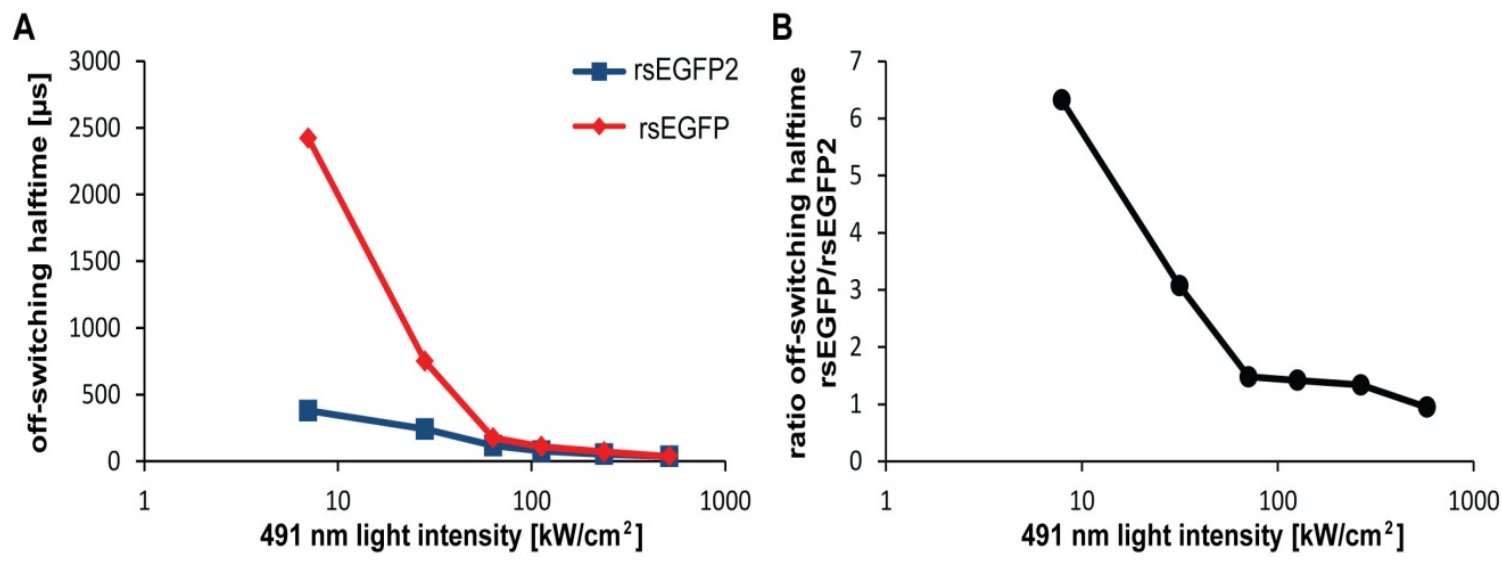

Figure IV.29: Comparison of off-switching halftimes of rsEGFP and rsEGFP2 at different $491 \mathrm{~nm}$ light intensities. Switching was performed in living PtK2 cells expressing VimentinrsEGFP and -rsEGFP2 protein. RSFPs were switched into the on-state by irradiation with 1.6 $\mathrm{kW} / \mathrm{cm}^{2}$ intense $405 \mathrm{~nm}$ light for $50 \mu \mathrm{s}$. Off-switching with light of $491 \mathrm{~nm}$ was performed at different light intensities. (A) Measured off-switching halftimes of rsEGFP and rsEGFP2. (B) Speed differences between rsEGFP and rsEGFP2. 
switching fatigue of rsEGFP2 in comparison to rsEGFP (Figure IV.30).

Hence, the comparison of rsEGFP and rsEGFP2 demonstrated a clear enhancement in switching fatigue and switching speed under RESOLFT-like light intensities for rsEGFP2. This should allow a higher number of RESOLFT images of a structure to be taken, and should increase the imaging speed. In the following, this was verified by adapting the RESOLFT microscopy approach to rsEGFP2.

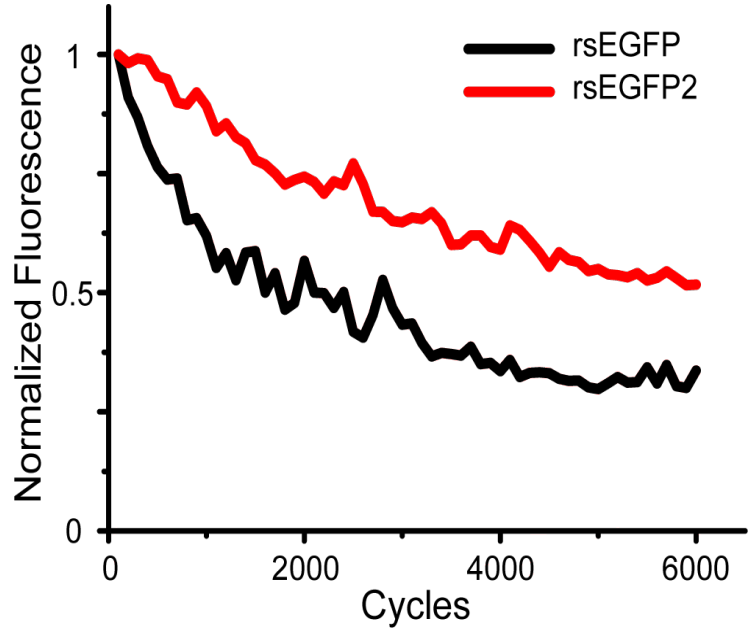

Figure IV.30: Switching fatigue of rsEGFP2 (red) and rsEGFP (black). Switching was performed by alternate irradiation with $405 \mathrm{~nm}$ $\left(1.8 \mathrm{~kW} / \mathrm{cm}^{2}\right)$ and $491 \mathrm{~nm}\left(1.3 \mathrm{~kW} / \mathrm{cm}^{2}\right)$ light. Illumination times were kept as short as possible to fully switch to the fluorescence maximum/minimum. Each data point is a average of 100 cycles.

\section{c. RESOLFT-type Microscopy Using rsEGFP2}

In a next step, the applicability of rsEGFP2 for RESOLFT microscopy was tested (imaging performed by Ilaria Testa). Vimentin-rsEGFP2 fusion protein was expressed in PtK2 cells. The RESOLFT irradiation sequence was adapted to the faster switching properties of rsEGFP2. To switch proteins into the fluorescent state, the sample was irradiated with $405 \mathrm{~nm}$ light $\left(1.5 \mathrm{~kW} / \mathrm{cm}^{2}\right)$ for $40 \mu \mathrm{s}$. After a short illumination break of $10 \mu \mathrm{s}$, the doughnut shaped $491 \mathrm{~nm}$ beam $\left(2 \mathrm{~kW} / \mathrm{cm}^{2}\right.$, $300 \mu \mathrm{s})$ was used to switch proteins in the periphery into the off-state. The residual on-proteins in the very center of the PSF were read-out with $491 \mathrm{~nm}$ light $\left(10 \mathrm{~kW} / \mathrm{cm}^{2}\right)$ for $30 \mu \mathrm{s}$. Finally a $20 \mu \mathrm{s}$ illumination break allowed the stage to move to the next position. This sequence was repeated at each scanning position. To further increase imaging speed, the switching sequence was done without any illumination break after on-switching. Furthermore, the scanning was performed in a classical linear way and not in the hop scanning mode, as was used for rsEGFP. The images were further optimized by a Richardson-Lucy deconvolution. Imaging of the Vimentin-rsEGFP2 fusion protein in living PtK2 cells (kept in a temperature-controlled chamber at $\sim 35^{\circ} \mathrm{C}$ ) revealed RESOLFT images displaying much thinner structures than classical confocal microscopy 
showed (Figure IV.31). The RESOLFT images had an overall size of $100 \mu \mathrm{m}^{2}$ and a pixel size of $20 \mathrm{~nm} \times 20 \mathrm{~nm}$. Hence the recording time for one image frame was $\sim 100 \mathrm{~s}$, which was $\sim 50$ times faster than the recording would have taken by using rsEGFP. The low switching fatigue of rsEGFP2 allowed the recording of 20 images of the same structure until the fluorescence signal was bleached to $\sim 70$ $\%$ indicating that it would be possible to record a further multiplicity of images. The time lapse RESOLFT imaging revealed fine structural changes in the Vimentin filaments (Figure IV.31).
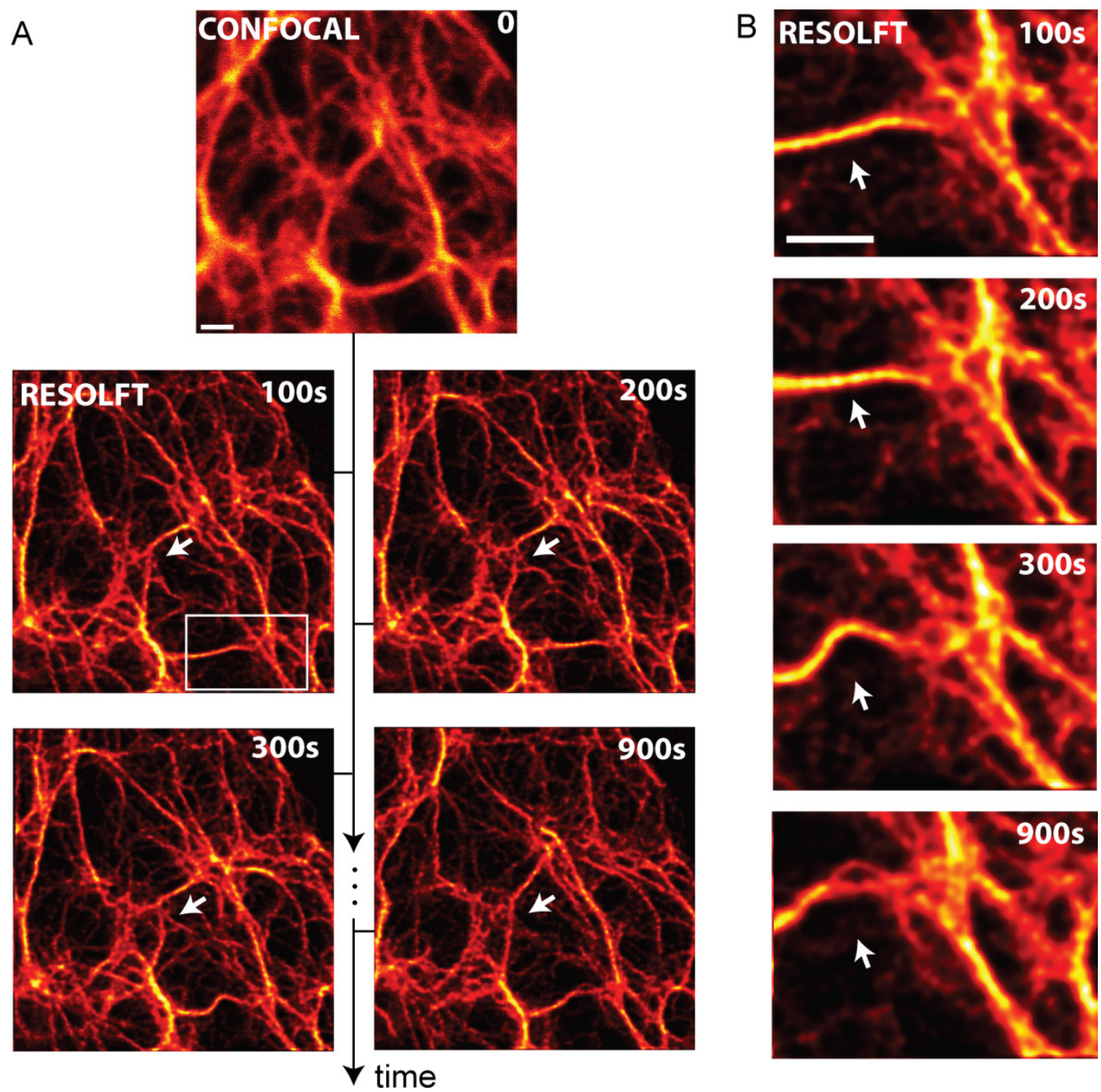

Figure IV.31: RESOLFT imaging of Vimentin-rsEGFP2 in Ptk2 cells. Vimentin filaments were imaged each $100 \mathrm{~s}$. A Richardson Lucy deconvolution was applied for all images. (A) Whole confocal and RESOLFT images. (B) Close up of the image area marked by the white frame in (A). Structural changes over time are indicated by white arrows. Scalebar: $1 \mu \mathrm{m}$. 

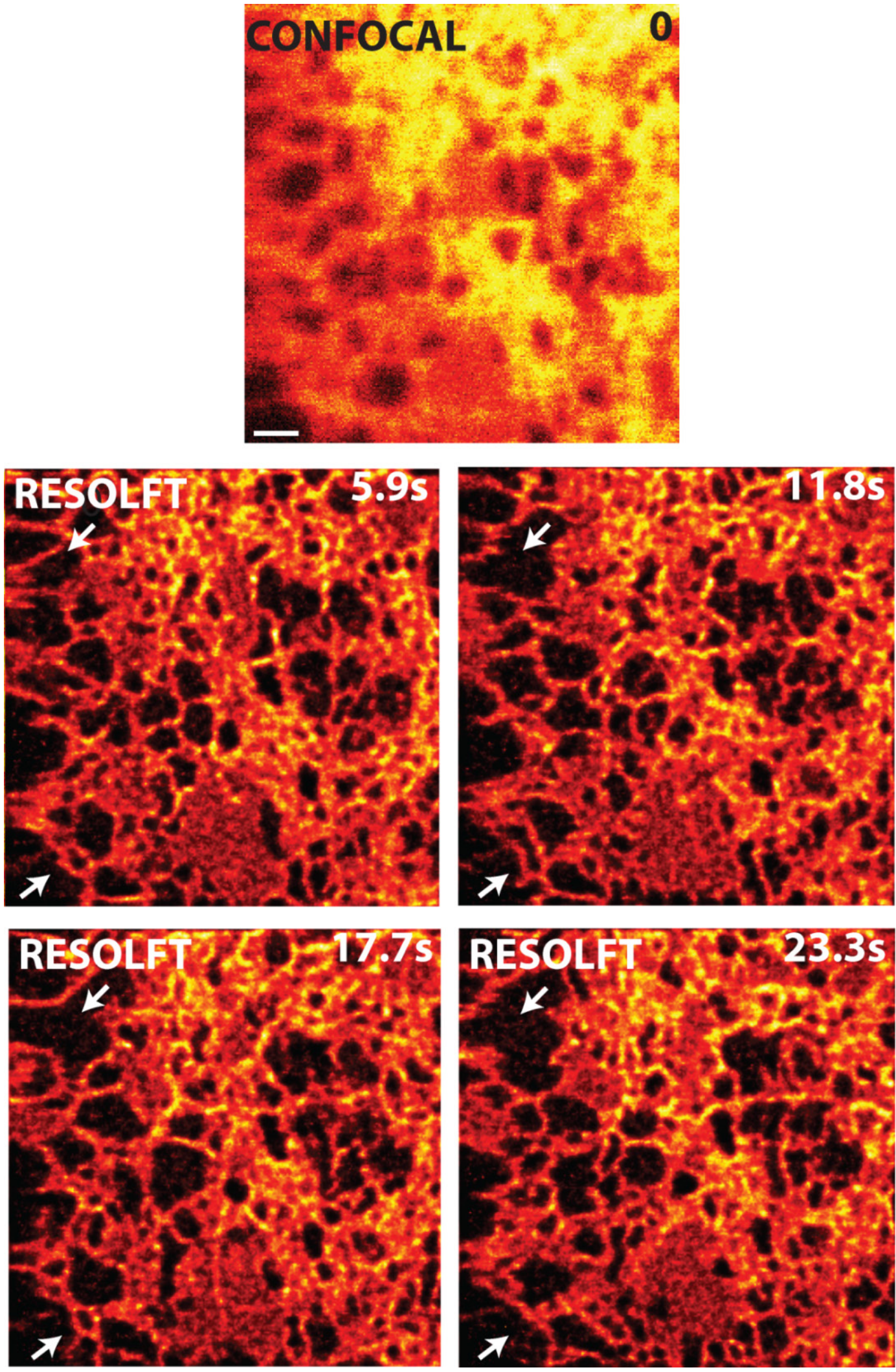

Figure IV.32: RESOLFT imaging of PtK2 cells ER using rsEGFP2 as volume stain. Confocal (top) and RESOLFT images (bottom) were recorded on the same area with a repetition rate of 1 frame per $5.9 \mathrm{~s}$. Images were interpolated to a pixel size of $20 \mathrm{~nm} \times 20 \mathrm{~nm}$ and a Richardson Lucy deconvolution was applied. Fast changes in the ER structures are exemplarily highlighted by the white arrows. Scale bar: $1 \mu \mathrm{m}$ 
To determine the usability of rsEGFP2 in imaging of faster moving structures, rsEGFP2 was fused to the ER retention signal. Expression in PtK2 cells resulted in a volume staining of the ER with diffusing rsEGFP2 proteins (Dayel, M.J. et al. 1999). RESOLFT imaging was performed with a pixel size of: $40 \mathrm{~nm} \times 40 \mathrm{~nm}$. The RESOLFT switching sequence was adapted to $20 \mu$ s for on-switching (3 $\left.4.5 \mathrm{~kW} / \mathrm{cm}^{2}\right), 50 \mu \mathrm{s}$ for off-switching $\left(6-10 \mathrm{~kW} / \mathrm{cm}^{2}\right)$ and $5 \mu \mathrm{s}$ for read-out (30 $50 \mathrm{~kW} / \mathrm{cm}^{2}$ ). This allowed imaging of $100 \mu \mathrm{m}^{2}$ in $5.9 \mathrm{~s}$. Although the rsEGFP2 fusion was freely moving inside the ER, a resolution enhancement was clearly achieved with RESOLFT imaging, showing thinner ER structures in comparison to conventional confocal microscopy (Figure IV.32). Repeated imaging revealed fast changes in the ER structure. During the whole experiment (37 images in total) the cell stayed viable, indicated by an intact ER network.

To further decrease the image recording time the image size was decreased. This enabled RESOLFT imaging of ER structures with a repetition rate of 2 Hertz (Figure IV.33.A). Slight changes in the ER structures could be detected. This indicates that an imaging interval of 2 Hertz is sufficient to detect ER movements in detail. As a second sample the Pex16-rsEGFP2 fusion protein was expressed in PtK2 cells. RESOLFT microscopy with a imaging speed of 2 Hertz revealed fast moving peroxisomal vesicles (Figure IV.33.B).

When using rsEGFP in neuronal brain slices (chapter IV.3.d) RESOLFT imaging was limited to small areas like single spines to avoid very long recording times.

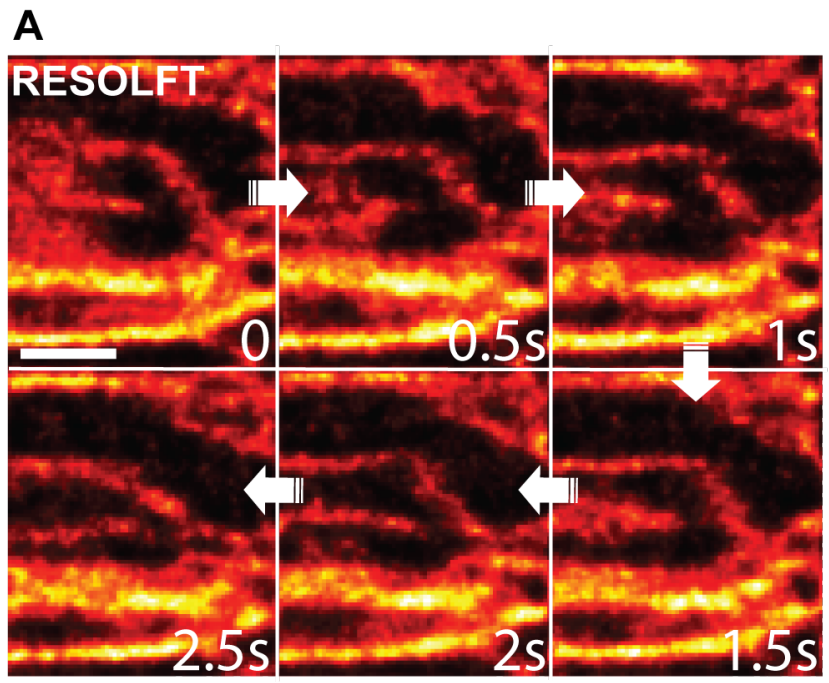

B

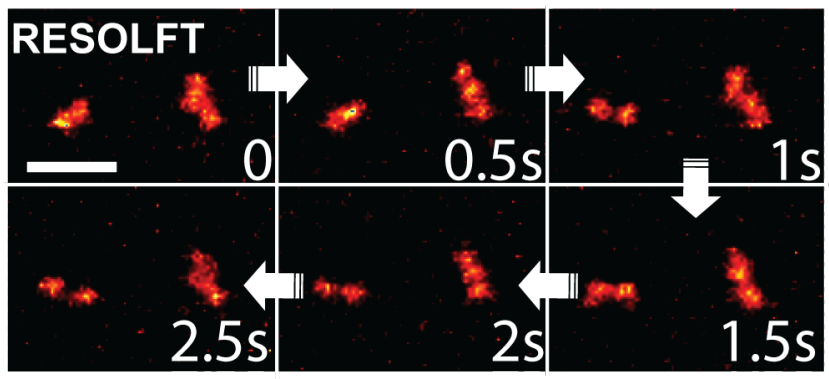

Figure IV.33: RESOLFT time lapse imaging at 2 Hertz. (A) image-series of ER structures in PtK2 cells. (B) Peroxisomal vesicles division and fusion in PtK2 cells. Scalebars: $1 \mu \mathrm{m}$ 
This experiment was repeated by using a lifeact-rsEGFP2 construct (onswitching: $40 \mu \mathrm{s} ; 1.6 \mathrm{~kW} / \mathrm{cm}^{2} /$ off-switching: $400 \mu \mathrm{s} ; 2 \mathrm{~kW} / \mathrm{cm}^{2} /$ read-out: $40 \mu \mathrm{s}$; $10 \mathrm{~kW} / \mathrm{cm}^{2}$ ). To additionally gain resolution in the axial direction a second offswitching beam path with a z-phaseplate was added. The increased imaging speed in RESOLFT microscopy achieved by using rsEGFP2 allowed for the recording of larger field of view images $\left(140 \mu \mathrm{m}^{2}\right)$ of dendrite cells in living organotypic hippocampal brain slices of mice (Figure IV.34). While all spines along the dendrite appeared blurred in the confocal image, RESOLFT microscopy revealed smaller spine necks and different spine head shapes. This made it easier to discern single spines from each other. A spine neck diameter of $<80 \mathrm{~nm}$ was detected indicating resolutions comparable to the recorded rsEGFP images.
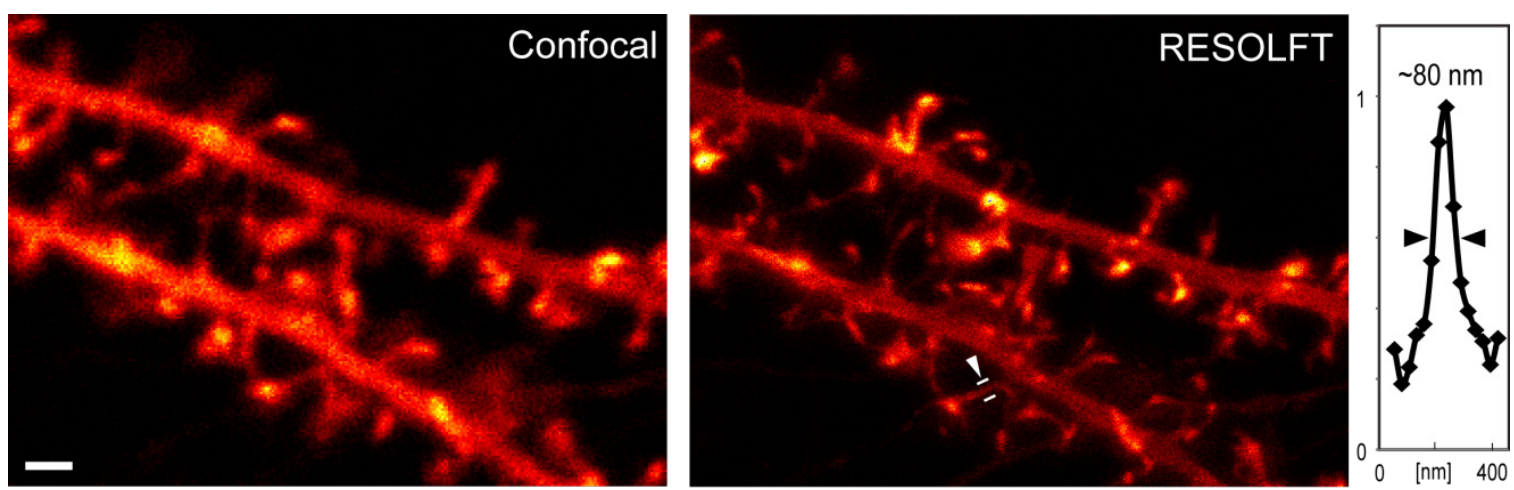

Figure IV.34: RESOLFT microscopy of living organotypic hippocampal mice slice expressing lifeact-rsEGFP2. The graph shows the normalized fluorescence profile between the two white markers with the white arrowhead indicating the direction. Scalebar $1 \mu \mathrm{m}$

Hence, rsEGFP2 allows for repeated RESOLFT measurements of living cells and tissue with subdiffraction resolution. This was demonstrated while utilizing low light intensities in the $\mathrm{kW} / \mathrm{cm}^{2}$ range. In comparison to rsEGFP, it enabled faster RESOLFT imaging due to its enhanced switching kinetics. Thus, faster moving structures were also imaged successfully. 


\section{Discussion}

Since the discovery of GFP, FPs became an indispensable and widely used tool in life sciences. These proteins allowed real time analyses of protein localizations and dynamic changes in the living cell, thereby reviving fluorescence microscopy. In the past few years, the novel FP-subgroup of RSFP has got more and more attention. The ability to reversibly switch RSFPs between a fluorescent and nonfluorescent state bears high potential for use in a variety of applications like protein tracking (Chudakov, D.M. et al. 2006), data storage (Adam, V. et al. 2010), and, most notably, randomly- and targeted switching-based superresolution microscopy approaches (Hell, S.W. 2007; Hell, S.W. 2009; Patterson, G. et al. 2010). While single molecule switching microscopy utilizing photoactivatable or reversibly switchable fluorescent proteins is well established (Egner, A. et al. 2007), RSFP-based RESOLFT-type microscopy was limited to a few proof-of-principle experiments due to lack of RSFPs providing the necessary characteristics (Hofmann, M. et al. 2005; Dedecker, P. et al. 2007).

The rsEGFP generated in this work provides these characteristics required for RESOLFT applications (Grotjohann, T. et al. 2011). Reduced switching fatigue, fast switching speed, and low residual fluorescence in the off-state allow live cell RESOLFT imaging for the first time. Here, light intensities in the $\mathrm{kW} / \mathrm{cm}^{2}$ range are used which are comparable to those used in CLSM and 1,000,000 fold lower than the intensities used in STED microscopy. This should strongly reduce the phototoxic effects in comparison to STED microscopy and should hence make RSFP-based RESOLFT microscopy to the sub-diffraction microscopy method of choice when living samples are imaged. The faster switching variant rsEGFP2 facilitates even faster imaging speeds and thus allows imaging of fast moving structures.

The reduced switching fatigue and enhanced switching speed also displays strong advantages in data storage applications, which was exemplarily demonstrated in this work by sequentially storing the text of a whole book into a protein layer in $>6000$ writing and reading cycles. Furthermore, the possibility to write and read data with spots smaller in sizes than the diffraction limit of light by adapting the RESOLFT principle allows the spots to be written in closer proximity to achieve higher storage densities. 


\section{A Comparison of Screening Approaches Utilized for RSFP Generation}

The multiplicity of candidate proteins derived from a semi-rational approach necessitates the establishment of a screening system to quantify the complex switching characteristics of RSFPs.

The commonly used screening approach to generate FPs is FACS screening (Cormack, B.P. et al. 1996; Shaner, N.C. et al. 2004; Subach, O.M. et al. 2008) providing a way to screen on a high throughput level for protein brightness and spectral characteristics. However, these high speed sorter demand short interaction times in the microsecond range. Hence estimation of the switching kinetics (usually in the millisecond time range) as well as the determination of the on- and the off-state on a single cell level is impossible and thus excludes the possibility to effectively screen for RSFP variants exhibiting defined switching characteristics.

A more promising approach for screening the switching characteristics of RSFPs is the use of an automated epifluorescence microscope to scan agar plates with E. coli colonies expressing the RSFPs and to apply a defined illumination sequences to detect the switching characteristics. Such systems were already used to identify and characterize improved RSFPs (Stiel, A.C. et al. 2007; Andresen, M. et al. 2008). These setups use mercury lamps as light sources, which only allow illumination intensities in the $\sim \mathrm{W} / \mathrm{cm}^{2}$ range. The determination of bleaching characteristics and switching fatigue with such screening systems may be difficult because measuring the bleaching kinetics under such light intensities would take a relatively long time leading to a very low throughput. A possible way to overcome this limitation in throughput may be the detection of many bleaching kinetics at low light intensities in parallel by illuminating many $E$. coli colonies at once and utilizing stronger lamp light sources (Shaner, N.C. et al. 2008).

As already mentioned, it is mandatory to screen for enhanced protein variants under the same light intensities, as it is used in the later application, due to nonlinear effects of light intensities on protein characteristics (see Chapters II.1.a ,IV.1, andV.1.a). In comparison to all mentioned screening approaches, the here 
reported screening setup is the only system which combines the possibility to determine switching characteristics and to screen with light intensities in the $\mathrm{kW} / \mathrm{cm}^{2}$-range. Hence, it gives a detailed insight how an RSFP reacts at RESOLFT-like light intensities.

The relatively complex and long lasting switching sequence on each $E$. coli colony is at the cost of the screening speed. Moreover, the small foci sizes used in the here presented screening approach made the implementation of an autofocus necessary which roughly takes the same time as the illumination sequence. This results in relatively long dwell times and low throughput. Hence, in comparison to conventional screening methods like for example FACS, this screening system can be seen as a high quality and low quantity approach. To enhance the throughput, a combined use with other high throughput screening systems are imaginable like pre-sorting the $E$. coli samples by FACS and then subsequently screening selected variants by the approach presented here.

A further limitation of the established screening setup is the time resolution of the data acquisition which lies in the millisecond range. In fact, with the identification of the fast switching rsEGFP2, the time resolution was already reached. Hence it is not possible to discern faster switching variants from rsEGFP2 with the established screening system. The time limitation is due to the acquisition software. An optimization of the software should easily allow higher time resolutions and, in consequence, screening for faster switching variants which would allow even faster RESOLFT imaging.

Overall, the established screening system fills a gap in the group of actually known screening approaches by presenting a viable approach to screen RSFP characteristics and switching kinetics at $\mathrm{kW} / \mathrm{cm}^{2}$ range light intensities. Such light intensities may make this screening approach not only favorable to screen for RESOLFT-RSFPs, but also to generally screen for FPs utilized in applications using $\mathrm{kW} / \mathrm{cm}^{2}$-range light intensities like for example, improving the photostability of FPs for CLSM. 


\section{Introduction of Fast, Persisting, and Reversible Switching into EGFP}

This work focuses on the generation of RSFPs with optimized characteristics like high brightness, fast and efficient switching, and, most notably, high photostability and low switching fatigue. Taking in account that FPs can be converted into RSFPs, like it was shown for several FPs previously (Ai, H.W. et al. 2006; Stiel, A.C. et al. 2008), the high photostability and brightness, the excellent tagging characteristics, its proven usability for a broad range of applications, and the detailed knowledge about this protein (Shaner, N.C. et al. 2005; Shaner, N.C. et al. 2007) made EGFP a favourable starting point to generate RSFPs for RESOLFT microscopy.

\section{a. The Non-Linear Dependence of Light Intensity on RSFP Characteristics}

A variety of different RSFPs, like Dronpa, Padron, rsCherryRev, and rsFastLime exhibit low residual fluorescence in the off-state of only a few percent (Ando, R. et al. 2004; Stiel, A.C. et al. 2007; Andresen, M. et al. 2008; Stiel, A.C. et al. 2008). These values were determined by using light intensities in the $\mathrm{W} / \mathrm{cm}^{2}$ range, and they can change when using more intense light (see Chapter IV.2). At light intensities in the $\mathrm{kW} / \mathrm{cm}^{2}$ range, Dronpa, Padron, and rsCherryRev exhibit a strongly increased residual fluorescence in the off-state ( $\geq 10 \%)$. Interestingly, the fluorescence background in the off-state of rsFastLime stays low. This indicates non-linear effect of the light intensity on RSFP characteristics. Such non-linearity effects not only affect the residual fluorescence in the off-state. For example, rsFastLime exhibits stronger switching fatigue under higher light intensities (see Chapter IV.2). Also the bleaching characteristics of FPs may vary when using light of different intensities. mOrange2 is more photostable than EGFP under mercury lamp illumination, but under CLSM-like light intensities, EGFP is more stable (Shaner, N.C. et al. 2008). Hence, such light intensity dependent nonlinear effects seem to appear in a variety of different FP and RSFP characteristics. They may result from changes in the probability to address different excitation states and deexcitation pathways. For example, bleaching 
under low intense light mainly appears from the $S_{1}$ and $T_{1}$ states. At higher light intensities, the probability of population of higher excited states, which exhibits different bleaching rates, increases. The probability to populate higher excited states as well as the different bleaching rates of these states are fluorophore specific, and may be the reason for the appearance of non-linear effects of light intensities on the FP characteristics. Accordingly, it is important to characterize the FPs under the same light intensities at which they are to be used in the final approach (see also Chapter V.1).

Taking this into account, the rsEGFP and rsEGFP2 characteristics are unique at light intensities in the $\mathrm{kW} / \mathrm{cm}^{2}$ range. Dronpa-M159T (Stiel, A.C. et al. 2007) is the only known RSFP, which exhibits comparable switching speeds, fatigue and contrast under RESOLFT-like light intensities. However, the use of DronpaM159T for RESOLFT applications was limited to prove-of-principle experiments using highly concentrated and purified protein and was not used for live cell imaging (Dedecker, P. et al. 2007). Live cell RESOLFT imaging using DronpaM159T might be challenging due to its low brightness of $\sim 17 \%$ compared to Dronpa. A second protein initially used for RESOLFT proof-of-principle approaches is asFP595 (Lukyanov, K.A. et al. 2000; Hofmann, M. et al. 2005; Schwentker, M.A. et al. 2007). This protein also exhibits strong switching fatigue, thereby limiting its RESOLFT applicability. Further, asFP595 is a tetrameric protein excluding its use in live cell approaches.

Overall, the direct comparison of the rsEGFP- and the rsEGFP2-characteristics with those of other proteins indicates their unique properties at $\mathrm{kW} / \mathrm{cm}^{2}$-range light intensities.

\section{b. The Structural Basis of Switching in rsEGFP and rsEGFP2}

The switching of RSFPs is mainly influenced by the amino acids surrounding the chromophore, which may stabilize or destabilize the trans and cis chromophore conformation and influence the probability to isomerize (Andresen, M. et al. 2007; Stiel, A.C. et al. 2007). Indeed, all switching-relevant mutations of the rsEGFP variants are located in close proximity to the chromophore or its hypothetical 
trans conformation (Figure V.1). Moreover, most amino acid positions, which were finally mutated in rsEGFP and rsEGFP2, were already suggested to influence switching at the beginning of this work, due to their position in close proximity of the chromophore. Subsequently, the final mutations at these positions were found by targeted site-directed mutagenesis instead of extensive random mutagenesis. Hence the structural knowledge about avGFP (Ormo, M. et al. 1996) allowed to generate rsEGFP and rsEGFP2 much more targeted and efficient.

The appearance of mutations at the hypothetical trans-side of the chromophore (Figure V.1) point clearly to a switching mechanism based on a cis-trans isomerization, which was already shown for most other RSFPs (Andresen, M. et al. 2005; Andresen, M. et al. 2007; Henderson, J.N. et al. 2007; Adam, V. et al. 2008; Brakemann, T. et al. 2010). In all these reported cases, the chromophore in the fluorescent state adopts the cis conformation, while the trans conformation represents the off-state.

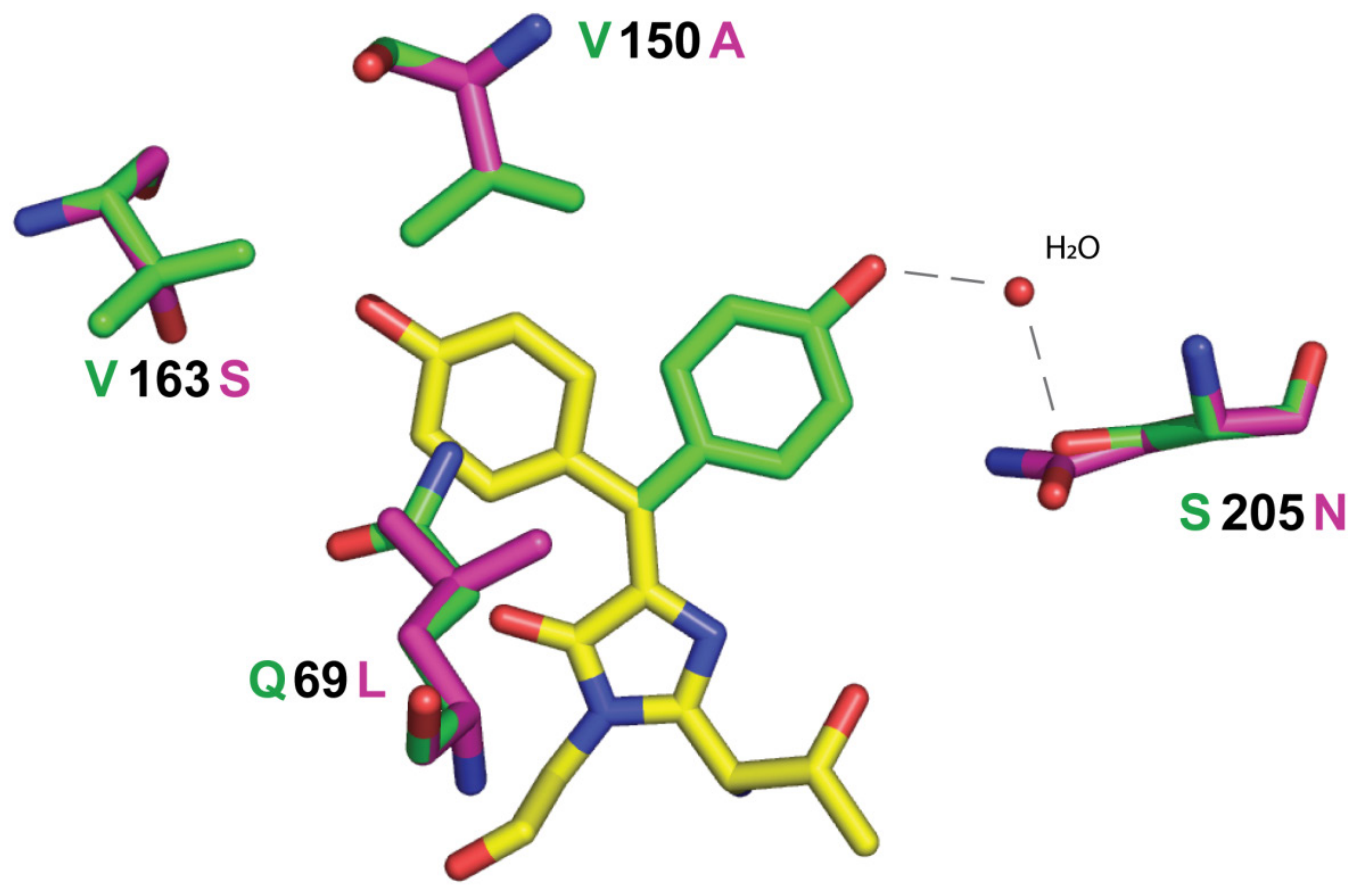

Figure V.1: Mutations of rsEGFP. EGFP amino acids and chromophor are marked in green (Royant, A. and Noirclerc-Savoye, M. 2011). rsEGFPs mutations in hypothetical conformations are shown in violet. The yellow off-state chromophor adapts a hypothetical trans conformation. 
The introduction of the single mutation Q69L already made mEGFP reversibly switchable. Gln69 is localized on the central $\alpha$-helix above the EGFP chromophore (Figure V.1). It is proposed to be involved in the water network surrounding the avGFP chromophore (van Thor, J.J. et al. 2005). Further, GIn69 seems to stabilize the radiative state of the excited chromophore of avGFP (van Thor, J.J. and Sage, J.T. 2006). The Q69L mutation disturbs this stabilization (van Thor, J.J. et al. 2005). This may allow alternative non-radiative deexcitation pathways like photoisomerisation. Furthermore, the longer carbon chain of the glutamine residue in comparison to the one of leucine is likely to sterically hinder the adoption of a trans chromophore formation.

Also the single mutation T65A makes EGFP already reversibly switchable. The introduction of a threonine at position 65 in GFP was one of the key mutations in generating EGFP (Heim, R. et al. 1995). In addition to the enhancement of the brightness and the decrease in maturation time, this mutation resulted in a completely deprotonated chromophore causing a single absorption peak at $\sim 490$ $\mathrm{nm}$ (see Chapter II.2). It was found to be due to the interruption of the hydrogen bond between Ser65 and Glu222 (see chapter II.2.b). However, besides Thr65, also alanine as well as glycine, cysteine, and leucine at this position interrupt these hydrogen bond (Delagrave, S. et al. 1995; Heim, R. et al. 1995; Cormack, B.P. et al. 1996) and yield the same single absorption peak at $\sim 490 \mathrm{~nm}$. Furthermore, it is known that the first chromophoric amino acid has an influence on the switching potential of RSFPs due to its ability to (de-/)stabilize the chromophore (Nam, K.H. et al. 2007). The resulting change in flexibility of the chromophore may influence the possibility to isomerize. Thus the switching ability introduced by T65A may be due to a destabilization of the chromophore at its basal side.

The exchange of Val163 into a serine in EGFP-(T65A, Q69L, A206K) improves switching by enhancing the switching speed and reduced the residual fluorescence of the off-state. A possible reason for this effect could be a stabilization of the chromophore in the trans conformation by the hydrogen bond between the serine and the apical hydroxyl group of the chromophore.

The introduction of the mutation V150A into EGFP-(Q69L, V163S, A206K) further enhanced the switching characteristics. Val150 lies in close proximity of the 
hypothetical trans position of the chromophore. The smaller alanine residue should further facilitate a chromophore isomerization reaction. Such a switching enhancing effect by removing sterical hindrances for isomerization was already reported for Dronpa (Stiel, A.C. et al. 2007).

Ser205 is known to play an important role in the chromophore stabilizing hydrogen network of avGFP (see Chapter II.2.b)(Erez, Y. et al. 2011). The exchange of Ser205 against an asparagine strongly enhances the off-switching speed, accompanied by a slight reduction in fluorescence brightness of the onstate. This may indicate a slight destabilization of the on-state chromophore resulting in a reduction of the activation energy barrier of photoisomerization. In other words, the isomerization may be more favored than a deexcitation by emitting a photon in this variant. F. Beltram and co-workers reported that the single mutation $\mathrm{E} 222 \mathrm{Q}$ is a key mutation for making avGFP derived FPs reversibly switchable (Bizzarri, R. et al. 2010). Glu222 is directly involved in the hydrogen bonding network surrounding the chromophore, as well as Ser205. Hence this result is in line with the discoveries presented in this work.

Interestingly, a combination of E222Q with those mutations of the rsEGFP variants was unfavorable with respect to the switching characteristics. Also the rsEGFP mutations V150A and S205N did not show any enhancement in switching characteristics after introducing them into rsEGFP2. This indicates that merging all switching mutations per se does not make an FP to an optimal RSFP, it is a more complex interplay of all mutations defining the switching characteristics.

However, a detailed understanding of the single mutations effects and their interplay make further structural analysis, such as the determination of the protein structure in the on- and off-state by X-ray crystallography, necessary.

\section{c. The Protonation States}

In order to switch the RSFPs with light of two different wavelengths they need to exhibit two different absorption peaks. These peaks are realized by the two different protonation states of the chromophore (Ando, R. et al. 2004; Andresen, M. et al. 2005; Andresen, M. et al. 2007; Stiel, A.C. et al. 2007). The protonated GFP-type chromophore absorbs at $\sim 400 \mathrm{~nm}$ and the deprotonated one at $\sim 490$ 
$\mathrm{nm}$. The on-state necessitates chromophores in the deprotonated state, which can emit fluorescent light after excitation. In case of positive switching RSFPs, the on-state chromophores partially adapt the protonated state, which allows to induce off-switching with light of shorter wavelengths compared to the fluorescence excitation light (Brakemann, T. et al. 2010). The off-state chromophore of an RSFP can adopt both protonation states. At least one absorption peak is required in the off-state. It is the protonated one in negative switching and the deprotonated one in positive switching RSFPs.

Although it is not mandatory for a negative switching RSFP to exhibit different single absorption peaks in the two fluorescent states, it may be desirable for an enhanced fast switching variant. Indeed, in the on-state rsEGFP and rsEGFP2 the chromophores are completely deprotonated and in the off-state mainly protonated (Figure IV.4.B and Figure IV.23.B). Because all chromophores in the on-state are deprotonated, all proteins can be immediately excited to fluoresce or to switch off. This results in a high ensemble brightness and fast off-switching. In line with this, the mainly protonated off-state chromophores of rsEGFP and rsEGFP2 allow fast on-switching.

In both rsEGFP variants just a very small fraction of the chromophores still adopt the deprotonated form in the off-state. This may be due to the fact that the transchromophore is partially deprotonated. Alternatively, not all of the RSFPs in the "off-state"-solution may be in the trans state. The off-switching light $(\sim 488 \mathrm{~nm})$ might to some degree also induce an on-switching from the off-state. This would lead to a mixed absorption spectrum consisting of a main off-state fraction and a small on-state fraction. This may also explain the existence of the residual fluorescence in the off-state. If this was to be the case, the residual fluorescence should decrease when using longer wavelengths of light for fluorescence excitation and off-switching. This has to be investigated in further studies.

\section{RESOLFT-type Microscopy Based on RSFPs}

With the invention of STED microscopy, a concept was presented which overcame the diffraction barrier of light with no theoretical limitation (Hell, S.W. and Wichmann, J. 1994). But STED is not the only way of switching fluorophores that can be used to enhance resolution. In theory, all light driven switching 
mechanisms allowing to discern between two fluorescent states can be used (Hell, S.W. 2003; Hell, S.W. et al. 2003). Examples for other switching mechanisms are ground state depletion (Hell, S.W. and Kroug, M. 1995) and the intrinsic switching characteristics of RSFPs (Hofmann, M. et al. 2005; Dedecker, P. et al. 2007; Schwentker, M.A. et al. 2007; Grotjohann, T. et al. 2011). Therefore, all these approaches are summed up to the so called RESOLFT microscopy.

\section{a. The Resolution of rsEGFP-RESOLFT Microscopy}

The here presented live cell RESOLFT microscopy is facilitated by the persisting switching characteristics of the rsEGFP variants, which can be switched $>1000$ times. This approach substantially overcomes the diffraction barrier reaching a resolution of less than $40 \mathrm{~nm}$. This is about the same resolution that was achieved for live cell STED microscopy using FPs $(<50 \mathrm{~nm})($ Hein, B. et al. 2008) and SNAP-tag staining with TMR $(<40 \mathrm{~nm})($ Hein, B. et al. 2010). The RESOLFT resolution was determined on the basis of line profiles across recorded keratin filaments in the living cell. It is unclear whether the resolution determination was affected by the structural size or by movements of the filament. Therefore the mentioned $40 \mathrm{~nm}$ resolution can be rather seen as the upper limit of the achievable resolution. To determine the resolution more precisely, further measurements need to be performed. This would benefit from an rsEGFP- or rsEGFP2-marked sample providing substantially smaller and immobilized structures.

The RESOLFT approach is a super-resolution microscopy technique with no theoretical limitation in resolution but, in practice, it is likely that the resolution is limited by optical perfection of the setup and the switching characteristics of the RSFP. An increase of resolution can be reached by switching more and more proteins in the periphery off, while the central area of on-state proteins becomes smaller and smaller. Hence the fluorescence signal of the center of the spot decreases while the off-state area (also emitting slight fluorescence, due to the residual fluorescence in the off-state of each RSFP) increases. Thus, decreasing the size of the central on-state spot also decreases the signal-to-noise ratio 
between inner and outer part of the focal spot, which finally limits the achievable resolution.

Hence, optimization of the rsEGFP variants for reduced residual fluorescence in the off-state and brightness may allow higher resolutions (Hofmann, M. et al. 2005; Schwentker, M.A. et al. 2007).

\section{b. Super-Resolution Microscopy at Low Levels of Light Using rSEGFP-RESOLFT}

The main advantage of RSFP-based RESOLFT microscopy is the low light intensity applied to the sample. The here presented RESOLFT images were recorded using a light intensity of $\sim 1 \mathrm{~kW} / \mathrm{cm}^{2}$. In contrast, STED microscopy uses intensities in the $\mathrm{MW} / \mathrm{cm}^{2}$ to $\mathrm{GW} / \mathrm{cm}^{2}$ range. Hence, RESOLFT uses light of up to $1,000,000$-fold less intensity than STED microscopy, due to the fact that the switching of RSFPs is based on toggling between two long lived ground states, and not on a modulation of the fluorescence capability by disallowing the population of a short, nanosecond lasting excitation state. Thus, in comparison to STED microscopy, the RSFP-based RESOLFT approach using low light intensities should strongly reduce phototoxic effects and should exclude the appearance of multi-photon induced damages (Hopt, A. and Neher, E. 2001). The risk of phototoxic effects should be comparable to those appearing in conventional CLSM, a widely used approach for live cell imaging, because CLSM uses the same light intensities as RESOLFT microscopy. In line with this, light induced damage was not detected within the here reported imaging experiments. However, they may appear after longer acquisition times and more often repeated imaging.

Especially UV and near-UV light is known to induce photodamage in living cells, due to its high energy and its ability to induce the production of reactive oxygen species (ROS) via photosensitizers (Peak, J.G. and Peak, M.J. 1990; Godley, B.F. et al. 2005). Thus, using light of $405 \mathrm{~nm}$ to switch RSFPs in living cells might be unfavorable. To further reduce phototoxic effects in RSFP-based RESOLFT microscopy, RSFPs should be generated which fulfill the requirements for this type of microscopy approach and absorb light of longer wavelength. Also two- 
photon excitation might be a way to get rid of the use of unfavorable short wavelengths of light.

Beside RSFP-RESOLFT, also the stochastical switching based super-resolution microscopy approach PALM facilitates intensities in the $\mathrm{kW} / \mathrm{cm}^{2}$ range (Hess, S.T. et al. 2006). However, this technique requires illumination of the whole imaging area for thousands of frames resulting in an overall illumination time of several seconds to minutes. In contrast, RESOLFT microscopy is a scanning approach with targeted illumination and a dwell time of $\sim 20 \mathrm{~ms}$ or shorter. As a result, the overall light dose applied to the sample is considerably lower than in PALM.

Hence, in comparison to all other FP-based live cell super-resolution methods which overcome the diffraction barrier of light without theoretical limitations, RSFP-RESOLFT microscopy applies the lowest energy to the sample. Thus, for imaging of living samples with resolutions below the diffraction limit, RSFP-based RESOLFT should be the microscopy approach of choice.

\section{c. Increasing the Imaging Speed in RSFP-based RESOLFT Microscopy}

Although the recording times of rsEGFP-RESOLFT presented in this work are still in the order of most super-resolution microscopy methods (Hell, S.W. 2007; Hell, S.W. 2009; Huang, B. et al. 2010), the dwell times of $\sim 20 \mathrm{~ms}$ are relatively slow in comparison to fast STED recordings (Westphal, V. et al. 2008). The dwell time in RSFP-RESOLFT is determined by the switching speed of the RSFP used, while switching affected by STED can be seen as instantaneously. To some extent, the switching speeds of an RSFP can be increased by applying higher light intensities. However, using light of much higher intensity may lead to changes in switching characteristics, as it has already been discussed in this work (see Chapter V.2.a). The limitation in shortening the dwell time may lead to problems when imaging fast moving structures, due to the fact, that a movement of the structure during switching may destroy the focal switching pattern. Hence, the RESOLFT imaging of faster moving structures necessitates the generation of faster switching RSFPs. The generation of faster switching proteins may be 
advantageous until a certain speed is reached, at which the number of emitted photons per switching cycles gets too weak for RESOLFT imaging. Note, the faster the switching characteristics of an RSFP of a given brightness, the less are the emitted number of photons per switching cycle. A promising candidate to overcome this limitation may be the recently generated RSFP Dreiklang (Brakemann, T. et al. 2011). Due to the fact, that in Dreiklang the switching is decoupled from the probing, the number of detectable photons per cycles is not limited. Hence, fast switching with high image contrast should be possible when Dreiklang could be optimized for switching fatigue and speed. Since it has been shown that Dreiklang can be used for RESOLFT imaging recently (Brakemann, T. et al. 2011) it is a promising candidate for such an approach.

In this work, a speed enhancement was realized by the generation of rsEGFP2, which switches up to $\sim 6.5$ times faster than rsEGFP. Further speed enhancement of rsEGFP2 could be achieved by increasing the light intensity by a factor of $\sim 10$. Taken together, rsEGFP2 samples can therefore be imaged $\sim 65$ times faster than the RESOLFT imaging of rsEGFP proteins took in this work (resulting dwell time: $\sim 300 \mu \mathrm{s}$ ). Using shorter dwell-time increases the imaging speed, but may not allow a complete off-switching of the proteins resulting in a higher fluorescence background in the periphery of the detection spot. Such backgrounds can be subsequently subtracted to some extant from the image by deconvolution (Hofmann, M. et al. 2005). The utilization of deconvolving methods finally allowed dwell times down to $95 \mu \mathrm{s}$, which was sufficient to image dendrites in a bigger field of view and recording of diffusing proteins in the ER (Dayel, M.J. et al. 1999).

However, for a detailed understanding of how fast the movement of proteins can get before the image resolution is influenced, further experiments and computational simulations need to be performed.

To further enhance the imaging speed of RSFP-based RESOLFT several adaptations of the RESOLFT setup could be tried out. One possibility is to adapt the microscope setup for multi-beam excitation and detection, as was already shown for STED microscopy (Bingen, P. et al. 2011). The high intensities needed for STED microscopy strongly limit the feasible number of beams due to the 
limited power of available lasers (Hell, S.W. and Andresen, V. 2001). In contrast, RESOLFT just uses powers in the low $\mu \mathrm{W}$-range per beam. Thus, already small and cheap laser sources of $10-50 \mathrm{~mW}$, which are normally used in conventional CLSM, should be sufficient to realize a multiple number of beams in parallel. Transferring the RESOLFT principle to a confocalized line scanning approach should further increase the imaging speed. By repeated recording of a sample with different line angles, a two-dimensional resolution enhancement should be achievable. In line with this, the fastest way of RESOLFT imaging might be facilitated by using a wide-field microscope approach, camera detection, and a pattern of zero-intensity lines, so-called structured illumination (Heintzmann, R. et al. 2002; Gustafsson, M.G. 2005; Schwentker, M.A. et al. 2007). Recently, such an approach was demonstrated using Dronpa (Rego, E.H. et al. 2011). Dronpa can only undergo enough switching cycles for this kind of approach when it is switched with very low light intensities of a few $W / \mathrm{cm}^{2}$. This led to very long switching and imaging times, and therefore required a fixation of the sample. However, the use of rsEGFP variants, which provide the required switching speeds and number of cycles, in an approach like this should allow fast RESOLFT imaging at several Hertz of in a large field of view.

Hence RSFP-RESOLFT fulfills all needs to become a fast imaging technique to study live cell processes at the nanoscale.

\section{d. The Adaptability of the RESOLFT Approach to Different Experimental Needs}

The different rsEGFP2-RESOLFT experiments, presented in this work, indicate that the RESOLFT switching scheme can be adapted to the requirements of the sample and the experimental needs. For example, by decreasing the onswitching time only, a subset of the proteins can be transferred into the on-state. This should enlarge the number effective switching cycles and images that can be performed, but at the cost of effective contrast and image brightness. The elongation of the probing time allows to collect more photons up to a maximum, which is determined by the absolute number of emitted photons per off-switching cycle by a negative switching RSFP. However, it also may decrease the achievable resolution because the readout light switches the proteins in the 
center of the spot off. Hence, the longer the probing takes, the lower is the relative ratio of center signal to background signal of the periphery.

Further, it is important to choose the right rsEGFP variant for a certain experiment. rsEGFP and rsEGFP2 possess a comparable brightness so that they emit the same number of photons per time. But a faster switching variant emits less photons per switching cycle than a slower one of the same brightness. Hence, rsEGFP2 is favorable for experiments requiring fast imaging, while rsEGFP may be beneficial when imaging sparsely labeled and dim structures.

However, to understand the interdependence of all relevant variables influencing the RESOLFT imaging performance in detail, further research is necessary.

\section{All Optical Data Storage Approaches Using}

\section{rsEGFP}

The use of switchable organic dyes like spiropyrans for data storage has already been discussed for a long time (Hirschberg, Y. 1956; Berkovic, G. et al. 2000; Irie, M. 2000; Kawata, S. and Kawata, Y. 2000; Yokoyama, Y. 2000; Irie, M. et al. 2002; Adam, V. et al. 2010). In 1998 it was first suggested to use photochromic FPs for optical memories (Tsien, R.Y. 1998), followed by proof-of-principle experiments with Dronpa, Kaede and IrisFP (Ando, R. et al. 2004; Adam, V. et al. 2010). However, switching fatigue and speed strongly limited their applicability owing to long writing/erasing processes and a small number of possible rewriting cycles.

\section{a. The Use of rsEGFP for Rewritable Data Storage}

\section{Applications}

Previous experiments demonstrated the utilization of RSFP for data storage by switching pictures and figures into layers of proteins. The here presented approach facilitated by the reduced switching fatigue and faster switching of rsEGFP was used to sequentially store real data sets in a binary code. This enables a direct comparison with other established optical storage media.

The individual data spots of $\sim 0.5 \mu \mathrm{m}$ diameter had a center-to-center spacing of $1 \mu \mathrm{m}$. This is comparable to the storage density of the DVD standard $(0.74 \mu \mathrm{m}$ 
pits). Conventional DVD-RWs/+RWs can be rewritten $500-1000$ times. Hence rsEGFP demonstrated $>6.6$ times more read/write cycles, while being bleached by only $\sim 35 \%$. Thus, it is likely that it should be possible to use the same rsEGFP layer for $\sim 15,000 \mathrm{read} /$ write cycles before too many proteins are irreversibly photobleached and the reading becomes unreliable.

By defining a simple threshold value, the data were read out with a bit error rate of $3.7 \cdot 10^{-6}$, which means 7 errors in total. The error rate can possibly be further reduced by improving the readout algorithm and by using different data encoding techniques, like EFMPlus (Immink, K.A.S. 1995). This may allow to use image data with less contrast, which would again increase the number of achievable read/write cycles.

To store the data of a whole book took $\sim 44$ hours, but the time determining step of this experiment was not the switching speed of rsEGFP, it was the relatively long imaging time for reading out the storage area with CLSM. By illuminating the whole area at once to erase the stored data, by increasing the $405 \mathrm{~nm}$ writing intensity into the $\mathrm{kW} / \mathrm{cm}^{2}$ range, and by reading out in a wide field approach using a camera the whole experiment should be performable in a few minutes.

However, there are still major limitations in using classical RSFPs for long time data storage. After switching, the RSFPs thermally relax back into their equilibrium state, which destroys the written spot pattern over time. The relaxation halftime of rsEGFPs is $\sim 23 \mathrm{~min}$ at room temperature. This is sufficient for the data storage experiment presented here. However, storing data for substantially longer time should be possible by decreasing the temperature of the layer. Other RSFPs, like Dronpa and Padron, exhibit higher thermal stability (Andresen, M. et al. 2008). Hence, generating RSFPs, which allow to store data over a longer period of time, seems possible.

A further limitation for the use of conventional RSFPs in data storage applications is the restricted number of reading processes that can be performed before the data spots are switched off by the read-out light. This effect becomes stronger when using faster switching RSFPs. This limitation in read-out cycles may be overcome by using the recently generated RSFP Dreiklang, in which the fluorescence excitation is completely decoupled from the switching (Brakemann, 
T. et al. 2011). Thus, the data could be repeatedly read-out without switching the proteins off.

All in all, the possibility to write/erase faster and more often using rsEGFP displays a step forward in using RSFPs for rewritable data storage approaches, although it still can not overcome the limitations in long time storage and read out cycles. However, a major advantage of the rsEGFP variants in the data storage field lies in its applicability for sub-diffractional writing.

\section{b. rsEGFP Enables Diffraction-Unlimited All-Optical Writing}

The diffraction barrier of light limits conventional optical data storage devices in their storage density. Thus, up to now, an increase in storage density was achieved by using light of shorter wavelength and lenses with higher numerical apertures, or by utilizing several storage layers. For example, Blue-ray discs are read-out with a $405 \mathrm{~nm}$ laser instead of $650 \mathrm{~nm}$ lasers like in DVDs, and Double/Dual Layer DVDs enable to write in two different focal planes. To overcome this limitation in storage density, it was suggested to use the RESOLFT principle for data storage applications (Hell, S.W. et al. 2003; Hell, S.W. 2004).Consequently, the RESOLFT-type approach was applied to lithographic approaches which enabled the writing of structures of size below the diffraction limit (Corredor, C.C. et al. 2006; Menon, R. and Smith, H.I. 2006; Fourkas, J.T. 2010). However, writing structures in closer proximity to increase the storage density was not demonstrated up to now. The here presented rsEGFP-based RESOLFT writing approach overcame the limitation in storage density by writing discernible spots in closer proximity than conventional focusing allows. This approach should open new ways in the field of data storage research, which was up to now dominated by concepts using much shorter wavelengths of electromagnetic radiation at which focusing becomes exceedingly difficult.

The writing of smaller spots was achieved by transferring the on-state rsEGFP proteins into a permanent bleached state. Thus this approach is a single writing technique and does not allow erasing and rewriting processes. However, in principle, the bleached state of such an approach can be replaced by any other state that is unaffected by the switching wavelengths and can be distinguished 
from the fluorescent state. One interesting candidate for such an approach may be the reversibly switchable and photoconvertable IrisFP (Adam, V. et al. 2008). The reversible switching in the green state could be used for RESOLFT-writing to switch a small on-state spot. Subsequently the proteins in the on-state could be converted into the red form. The RESOLFT microscopy-based readout could then be performed on the red reversibly switchable form. However, this would first necessitate the generation of an IrisFP variant that is capable of a RESOLFT-type switching and imaging in the green as well as the red form.

In RESOLFT microscopy the resolution and the effective PSF is mainly influenced by the contrast between on-state proteins in the center and off-state proteins in the periphery of the focal spot (see Chapter V.3.a). Analog to this, the size and shape of a spot created by RESOLFT writing is mainly influenced by the contrast, which is given by the specificity of transferring only the on-state proteins into the third state (e.g. bleached state) instead of transferring off-state proteins into this state.

Hence, to further decrease the spot size, RSFPs have to be generated, which enable a transfer into a third state with higher specificity, to reach a higher contrast than the one reported here of $\sim 3.5: 1$. Unfortunately also the photoconversion process of IrisFP (a protein which may be a hypothetical candidate for sub-diffractional writing, as mentioned before) may not be a process of very high specificity because photo-conversion as well as the switching of the green form is driven by light of $405 \mathrm{~nm}$. Thus, to estimate the applicability of IrisFP as a starting point for the generation of enhanced data storage RSFP variants, the achievable contrast needs to be investigated.

Taken together, an optimal RSFP for sub-diffraction resolution writing and reading should fulfill the required switching characteristics for RESOLFT applications and should further allow a light driven transfer from the on-state into a discernible third state. This transfer should be decoupled from the switching and the fluorescence excitation wavelengths. 


\section{Outlook}

This works presented two novel RSFP variants enabling sub-diffraction writing/reading and live cell RESOLFT microscopy which allowed imaging of a variety of samples at nanometer resolution. These results can be seen as a first of a multiplicity of steps in making RSFP-RESOLFT widely applicable.

The generation or the optimization of red fluorescent RSFPs in order to make them fulfill the RESOLFT requirements of low switching fatigue, fast switching, and low residual fluorescence in the off-state should be of great interest. In combination with an rsEGFP variant, this would allow two color imaging which would extend the applicability of RSFP-RESOLFT to further scientific fields, like for example co-localization studies. Moreover, in comparison to green fluorescent variants, red fluorescent RSFPs require an excitation with light of longer wavelength. This would hence be the first step to abandon the unfavorable nearUV-light for switching (see Chapter V.3.b).

This work utilized RSFPs with a negative switching mode to enable RESOLFT imaging. However, also RSFP with a positive switching mode should be applicable in such an approach. In these proteins, the probing wavelength differs from the off-switching wavelength. Hence the inevitable sequential switching in RESOLFT microscopy using negative switching RSFPs could be substituted against a scheme of simultaneous illumination with both wavelengths. This would make RESOLFT measurements easier to realize.

The imaging speed may be one of the most limiting factors in RSFP-based RESOLFT microscopy. A major focus should therefore be to increase these speeds. This could be realized by multi-beam set-ups or structured illumination approaches and by the generation of faster switching RSFPs, which is required to further decrease the imaging dwell times (see Chapter V.3.c).

In comparison to STED microscopy, major advantages of RSFP-RESOLFT are the considerably lower light intensities. The power-outputs of conventional lasers are sufficient to use two-photon-excitation for switching and fluorescence excitation in RSFP-based RESOLFT approaches. This would be of advantage because two-photon excitation allows higher penetration depth and excludes the utilization of unfavorable short wavelengths of light. Hence, 2-photon RESOLFT 
microscopy would be of great advantage for in vivo imaging of, for example, whole animals (Berning, S. et al. 2012) and would further reduce phototoxic effects. Further, the shape of a two-photon doughnut PSF differs from that of a single photon one. The two-photon PSF shows a larger zero-intensity area in the center and steeper increasing maxima in the periphery. This may have favorable effects on the effective RESOLFT PSF, which still has to be analyzed.

A further aim may be the optimization of RSFP-RESOLFT microscopy for 3D super-resolution imaging. A promising way to additionally achieve higher resolution in the z-dimension is the realization of an isoRESOLFT microscope by combining the here presented RESOLFT approach with the 4Pi-concept (Schmidt, R. et al. 2008; Hell, S.W. et al. 2009; Schmidt, R. et al. 2009). Such an approach would give a detailed three dimensional insight into living samples. Further, it would open up new ways for sub-diffraction all-optical writing, thereby allowing not only to store data in several layers (Adam, V. et al. 2010), but also to store them much more densely in all three dimensions.

The here presented RESOLFT approach, facilitated by the generation of RSFPs with improved characteristics, demonstrates not only a new viable superresolution method to study living samples and for sub-diffraction writing/reading, but also opens up a new field in nanoscopy which has the potential to be extended to a wide spectrum of scientific applications. 


\section{Material \& Methods}

\section{Cell and Tissue Cultures}

\section{a. E. coli Culturing and Transformation}

This work utilized the following $E$. coli strains:

- DH5a (Hanahan, D. 1983) for plasmid propagation and cloning

- BL21-CP-RIL (Stratgene, La Jolla, CA, USA) for protein expression

- SURE (Stratgene, La Jolla, CA, USA) and MC1000 (Casadaban, M.J. and Cohen, S.N. 1980) for expressing MreB-fusion proteins

- Top10 (Invitrogen, Carlsbad, Ca) for chromophore maturation experiments

E. coli cells were grown in LB-medium (Luria Bertani medium: 0.5\% (w/v) or yeast extract; $1 \%(\mathrm{w} / \mathrm{v})$ peptone; $0.5 \%(\mathrm{v} / \mathrm{v}) 1 \mathrm{~N} \mathrm{NaOH} ; 0.5 \%(\mathrm{w} / \mathrm{v}) \mathrm{NaCl})$ on agar plates (LB-medium with $2 \%(\mathrm{w} / \mathrm{v})$ agar) at $30{ }^{\circ} \mathrm{C}$ or $37{ }^{\circ} \mathrm{C}$. Liquid cultures were additionally shaked.

To measure the cell density the optical density at $600 \mathrm{~nm}$ wavelength $\left(O D_{600}\right)$ was determined using a plastic cuvette and the Eppendorf biophotometer (Eppendorf, Hamburg, Germany). For blanking pure LB-medium was used.

For specific selection ampicillin (Sigma Aldrich Chemie $\mathrm{GmbH}$, Munich, Germany) or kanamycin (Applichem, Darmstadt, Germany) with a final concentration of $50 \mu \mathrm{g} / \mathrm{ml}$ was added to the medium.

Transformation of $E$. coli cells was performed by electroporation. To generate electro-competent cells, an E. coli culture was grown into the logarithmic phase. When reaching an $\mathrm{OD}_{600}$ of $\sim 0.6$ the culture was kept for $30 \mathrm{~min}$ at $4^{\circ} \mathrm{C}$. Afterwards cells were peletted by centrifugation for $15 \mathrm{~min}$ at $4000 \mathrm{~g}$ and $4^{\circ} \mathrm{C}$ using a Sorvall RC-5B centrifuge (DuPont Instruments, Wilmington, DE, USA). The cell pellet was washed first for $2 \mathrm{~min}$ in ice cold water, and second in 1/50 volume $10 \%(\mathrm{w} / \mathrm{v})$ glycerol. Finally cells were resuspended in $1 \mathrm{ml} 10 \%(\mathrm{w} / \mathrm{v})$ glycerol, separated into $40 \mu \mathrm{l}$ aliquots, flash frozen in liquid nitrogen, and stored at $-80^{\circ} \mathrm{C}$.

For transformation aliquots were thawed one ice. Afterwards $20 \mathrm{ng}-40 \mathrm{ng}$ of plasmid DNA were added. After 5 min incubation at $4{ }^{\circ} \mathrm{C}$ the cell solution was 
transferred to a $2 \mathrm{~mm}$ thick electroporation cuvette. An electroporator $(2.5 \mathrm{kV}$, $200 \Omega, 20 \mu \mathrm{F}$ ) was used to apply a short voltage pulse. Afterwards $1 \mathrm{ml}$ LBmedium was added to the suspension and subsequently incubated for 1 hour at $37^{\circ} \mathrm{C}$ on a shaker. Finally the cells were plated on agar plates containing the respective antibiotic and incubated at $30{ }^{\circ} \mathrm{C}$ for 2 days (for protein expression using pQE31 plasmids) or at $37{ }^{\circ} \mathrm{C}$ over night (for cloning experiments).

\section{b. Mammalian Cell Culturing and Transfection}

The mammalian cell line used in this work was PtK2, which was derived from the kidney epithelium of the long-nosed Potoroo (Potorous tridactylis). The cell line was obtained from the group of Prof. Klaus Weber (Max Planck Institute of Biophysical Chemistry, Göttingen, Germany). PtK2 cells were cultured in DMEM (Invitrogen, Carlsbad, California) containing 5\% FBS (PAA, Pasching, Austria), 100 units $/ \mathrm{ml}$ streptomycin, $100 \mu \mathrm{g} / \mathrm{ml}$ penicillin (all Biochrom, Berlin, Germany), and $1 \mathrm{mM}$ pyruvate (Sigma, St. Louis, USA ). The cells were grown in an incubator at $37^{\circ} \mathrm{C}$ and $5 \% \mathrm{CO}_{2}$.

For transfection, cells were seeded on cover glasses in 6-well plates. After one day Nanofectin (PAA, Pasching, Austria) was used to transfect cells with plasmid DNA. According to the manufacturer's instructions DNA was mixed with the polymer solution. After $15-30$ min of incubation the mixture was added drop wise to the cells ( $3 \mu \mathrm{g}$ DNA per well in total). 24 hours later the transfection medium was replaced by fresh DMEM growth medium. Cells were imaged 24 $72 \mathrm{~h}$ after transfection, depending on the expressed fusion construct.

\section{c. Preparation, Culturing, and Viral Transfection of Organtypic Hippocampal Slices}

$350 \mu \mathrm{m}$ thick hippocampal slices were prepared from postnatal day 5-7 wild-type C57BL/6 mice, embedded in a plasma clot on $0.14 \mathrm{~mm}$ thick glass cover slips and incubated in a roller incubator at $35{ }^{\circ} \mathrm{C}$ (Gahwiler, B.H. et al. 1997). The age of the slice cultures used for experiments ranged from 12 to 42 days after the preparation. 
For viral transfection of the slices, a modified Semliki Forest Virus containing the pSCA-Lifeact-rsEGFP or pSCA-Lifeact-rsEGFP2 vector (for cloning see Chapter VI.2.d) was injected into the CA1 area of the slice cultures using a patch pipette connected to a pressure-generator (Picospritzer, Parker, Pine Brook, NJ)(DiCiommo, D.P. and Bremner, R. 1998).

\section{Cloning and Mutagenesis Strategies}

\section{a. Plasmid Propagation from E. coli cultures}

The isolation of plasmid DNA from $E$. coli samples was based on the alkaline lysis method (Birnboim, H.C. and Doly, J. 1979). This was performed by using the QIAprep Spin Mini- and MidiPrep Kits (Qiagen, Hilden, Germany) following the manufacturer's instructions.

\section{b. General Amplification Strategy for Cloning}

To amplify defined DNA sequences for cloning 50 - 100 ng template DNA was mixed with $1 \mu$ forward and $1 \mu$ reverse primer $(1 \mathrm{mM}), 1 \mu \mathrm{dNTP}$ solution $(2 \mathrm{mM}$ of each:. dATP, dGTP, dCTP, dTTP), $5 \mu \mathrm{l}$ 10× PCR buffer (100 mM Tris-HCl, 500

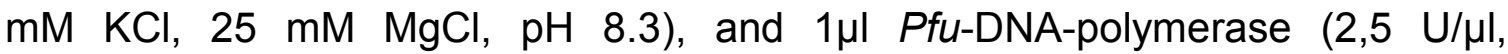
Fermentas, Burlington, Kanada). The mixture was added up to $50 \mu$ with water. The Amplification was performed with a TPersonal Thermocycler (Biometra, Göttingen, Germany) using the protocol displayed in Table IV.1.

Table VI.1: The thermocycler protocol for standard PCR amplification

\begin{tabular}{|l|l|l|}
\hline temperature $\left[{ }^{\circ} \mathrm{C}\right]$ & time $[\mathrm{s}]$ & repetitions \\
\hline 95 & 45 & $1 \times$ \\
\hline 95 & 45 & \\
55 & 60 & $30 \times$ \\
72 & 60 & \\
\hline 72 & 600 & $1 \times$ \\
\hline
\end{tabular}




\section{c. Cloning of Protein Expression Plasmids}

pQE31-EGFP: The coding sequence of EGFP (Clontech) was amplified (forward primer: GCTACGGATCCGATGGTGAGCAAGGGCGA / reverse primer: GCGCGAAGCTTTTACTTGTACAGCTCGTC) and ligated into the pQE31 expression vector (Qiagen, Hilden, Germany) using the BamHI and HindIII restriction sites.

pBad-rsEGFP and pBad-rsEGFP2: rsEGFP/rsEGFP2 was amplified (forward primer: AGGGCTCGAGCATGGTGAGCAAGGGCGAGGAG / reverse primer: CTTCGAATTCTTACTTGTACAGCTCGTCCAT) and cloned into the pBad vector (Invitrogen, Carlsbad, California) using the EcoRI and Xho/ restriction sites.

\section{d. Cloning of Fusion Proteins}

prsEGFP-MreB: This plasmid was generated by amplifying rsEGFP (forward primer: TACGAATTCAAGGAGATATACATATGGTGAGCAAGGGCGAGGAG । reverse primer: CATTCTAGACTTGTACAGCTCGTCCATG). Using the EcoRI and $\mathrm{Xbal}$ restriction sites, the YFP sequence in the pLE7 plasmid (Vats, P. and Rothfield, L. 2007) was replaced with the rsEGFP amplificat.

Fusion of rsEGFP and rsEGFP2 with Keratin19, Pex16, Vimentin, and histone H2B: To create fusion constructs of rsEGFP or rsEGFP2 with Keratin19, with the peroxisomal membrane protein Pex16, with Vimentin, or with the histone $\mathrm{H} 2 \mathrm{~B}$, rsEGFP variants were amplified (forward primer: GATCCACCGGTC GCGGCGTGAGCAAGGGCGAGGAGCTG / reverse primer: ACAACTTAAG AACAACAATTGTTACTTGTACAGCTCGTCCATGCC). The PCR fragments were cloned into the gateway destination vector pMD-tdEosFP-N (Testa, I. et al. 2008) using the restriction sites Agel and AflII. Hence the coding sequence of tdEosFP was replaced against the rsEGFP or rsEGFP2 sequence. The final plasmids pMD-Ker19-rsEGFP, pMD-Ker19-rsEGFP2, pMD-Pex16-rsEGFP, pMD-Pex16-rsEGFP2, pMD-Vim-rsEGFP, pMD-Vim-rsEGFP2, pMD-H2BrsEGFP, and pMD-H2B-rsEGFP2 were constructed by gateway vector conversion (Invitrogen, Carlsbad, CA) using the donor vectors pDONR223-Krt19, pDONR223-Pex16, pDONR223-Vim, and pDONR223-Hist1H2BN (Lamesch, P. et al. 2007). 
pMD-rsEGFP-Map2 and pMD-rsEGFP2-Map2: The plasmids pMD-rsEGFPMap2 and pMD-rsEGFP2-Map2, coding for a fusion protein of Map2 with rsEGFP and rsEGFP2, were generated by exchanging the $\alpha$-tubulin sequence of pEGFPTub (Clontech) against the Map2 sequence, amplified from pDONR223-Map2 (Lamesch, P. et al. 2007) using the restriction sites $\mathrm{Xhol}$ and BamHI. Subsequently, the EGFP sequence was exchanged against the PCR amplified sequences of rsEGFP and rsEGFP2, using the restriction sites Nhe/ and Bgl/l.

pMD-rsEGFP- $\alpha$-Tubulin: To generate pMD-rsEGFP- $\alpha$-Tubulin, the coding sequence of rsEGFP was amplified (forward primer: GATCCGCTAG CGCTAATGGTGAGCAAGGGCGAGGAG / reverse primer: CACTCGAGA TCTGAGTCCGGACTTGTACAGCTCGTCCATGCC) and exchanged against the EGFP sequence in pEGFP-Tub (Clontech) using the Nhe/ and Bgl// restriction sites.

pMD-Mito-rsEGFP: This plasmid, coding for a fusion protein of a mitochondrial targeting sequence and rsEGFP, was created by exchanging the DsRed1 sequence of pDsRed1-Mito (Clontech) against the one of rsEGFP amplified by PCR (forward primer: TCCACCGGTCGCCACCATGGTGAGCAAGGG CGAGGAG / reverse primer: GTCGCGGCCGTTACTTGTACAGCTCGTCCAT). The restriction sites Age/ and Notl were used.

Fusion of rsEGFP2 and the ER retention signal sequence: To generate the plasmid prsEGFP2-ER coding for the fusion construct of rsEGFP2 and the ER retention sequence rsEGFP2 was amplified (forward primer: CTGCAGGTCGACATGGTGAGCAAGGGCGAGGA / reverse primer: TTCTG CGGCCGCCTTGTACAGCTCGTCCATGCCGCCGGT). The PCR product was ligated into the pEF/myc/ER (Invitrogen, Carlsbad, Ca) using the Sal/ and Not/ restriction sites.

pSCA3-Lifeact-rsEGFP and pSCA3-Lifeact-rsEGFP2: The plasmid pSCA3Lifeact-rsEGFP and pSCA3-Lifeact-rsEGFP2 were cloned by replacing the YFP sequence of pSCA3-Lifeact-YFP (provided by Dr. Valentin Stein, MPI for Neurobiology, Munich, Germany) against rsEGFP and rsEGFP2. To this end, rsEGFP and rsEGFP2 were amplified (forward primer: TCCACCGGTCGC CACCATGGTGAGCAAGGGCGAGGAGCTGTTC / reverse primer: TACCCTGC GGCCGCTTTACTTGTACAGCTCGTCCATGCC) and cloned into the Age/ and Not/ restriction sites. 


\section{e. Site-Directed Mutagenesis}

To introduce mutations at a defined position the site directed mutagenesis approach was used. This approach is based on the utilization of two complementary primers of $\sim 20$ bps binding at the position where the mutation should be introduced. The mutated codon was localized at the center of the primers. This method was used to exchange a codon against another specific codon as well as against random codons. To this end, primers with random nucleotides were designed, which are marked with an $\mathrm{N}$ in the sequence.

$1 \mu \mathrm{l}$ of each primer $(10 \mu \mathrm{M})$ was mixed with $50-100 \mathrm{ng}$ template DNA (including the DNA sequence, which should be mutated), $5 \mu \mathrm{PCR}$ buffer (100 mM Tris$\mathrm{HCl}, 500 \mathrm{mM} \mathrm{KCl}, 25 \mathrm{mM} \mathrm{MgCl}, \mathrm{pH}$ 8.3), $1 \mu \mathrm{l}$ dNTPs (2 mM of each:. dATP, dGTP, dCTP, dTTP), and $1 \mu \mathrm{l} P f u$-DNA-polymerase $(2,5 \mathrm{U} / \mu \mathrm{l}$, Fermentas, Burlington, Kanada). Water was added to the mixture to a final volume of $50 \mu \mathrm{l}$. This mixture was used for a linear PCR reaction to amplify the whole template plasmid (Figure VI.1). The PCR protocol is shown in Table IV.2.

Afterwards $1 \mu \mathrm{l} D p n I$ was added to the PCR product, which was subsequently incubated at $37^{\circ} \mathrm{C}$ over night. This was a crucial step to get rid of the template DNA. The final linear DNA amplificate was transformed into E. coli cells. Finally, in the cell repairing mechanisms took place, leading to the final mutated plasmid (Figure VI.1).
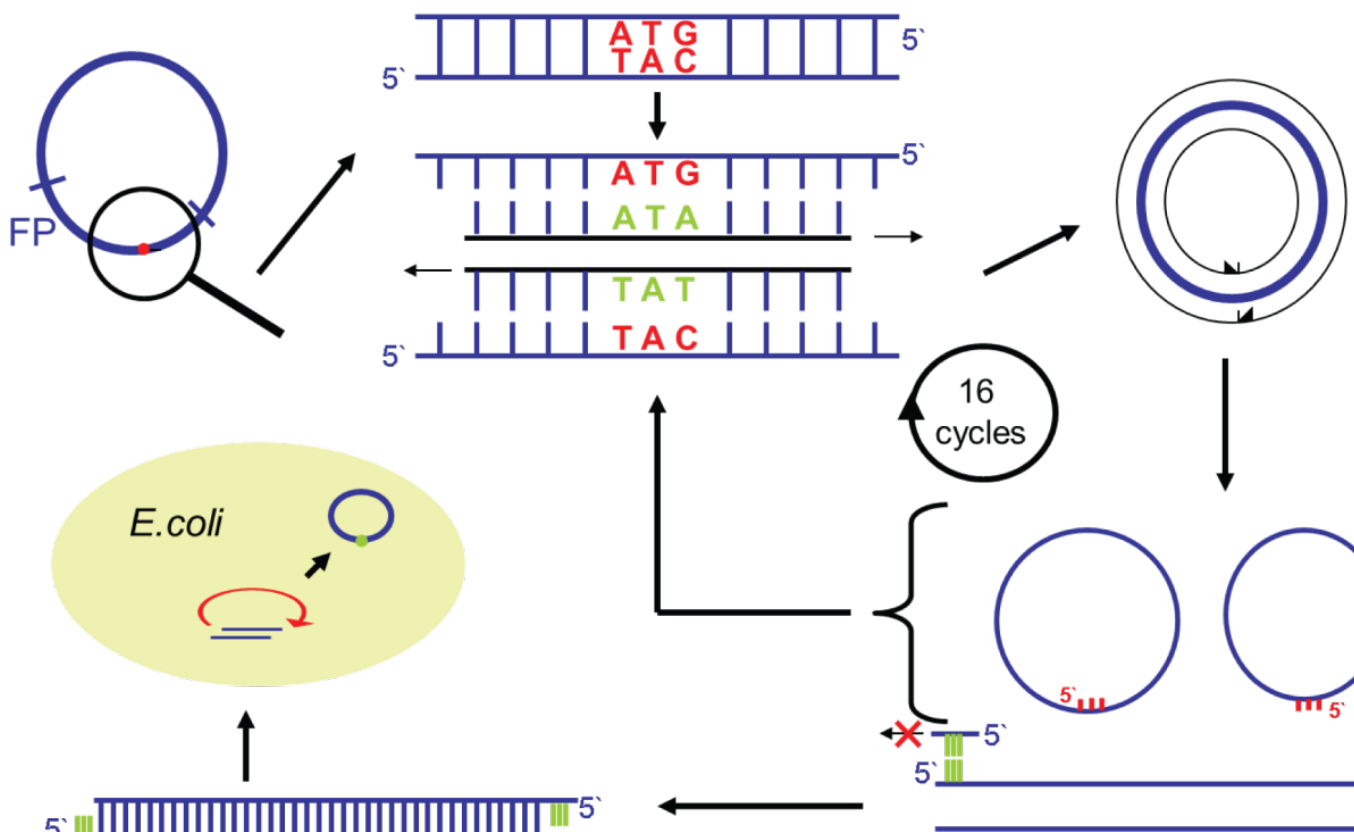

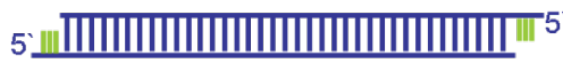

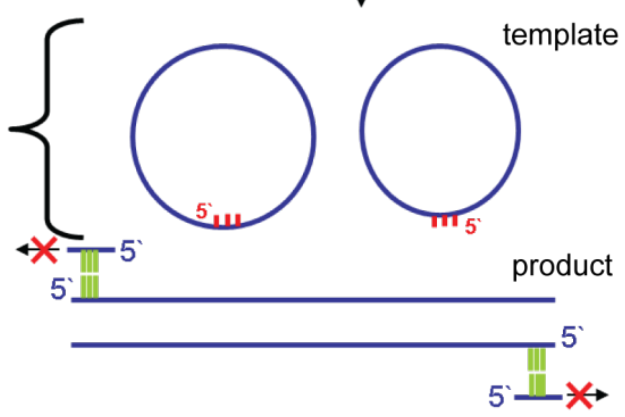

Figure VI.1: Schematic overview of the site-directed mutagenesis principle. 
Table VI.2: The thermocycler protocol for site-directed mutagenesis

\begin{tabular}{|l|l|l|}
\hline temperature $\left[{ }^{\circ} \mathrm{C}\right]$ & time $[\mathrm{s}]$ & repetitions \\
\hline 95 & 30 & $1 \times$ \\
\hline 95 & 60 & \\
55 & 60 & $16 \times$ \\
68 & 510 & \\
\hline 68 & 600 & $1 \times$ \\
\hline
\end{tabular}

\section{f. Error-Prone Mutagenesis}

To introduce random mutations into RSFPs error-prone mutagenesis was performed (Leung, D.W. et al. 1989). This method is based on an amplification of the RSFP coding DNA sequence combined with a single cloning step.

The random point mutations were introduced by forcing the polymerase to make mistakes during amplification. Therefore the Taq-polymerase was used, which has no proof-reading function. The probability of amplificational mistakes was raised by increasing the manganese concentration and by using unequal concentrations of the different dNTPs.

For the error-prone PCR reaction $1 \mu \mathrm{g}$ template-DNA, $10 \mu \mathrm{l}$ of each primer (10 $\mu \mathrm{M}$ stock solution), $10 \mu \mathrm{l} 10 \times$ error-prone dNTPs solution (2 mM dATP, $2 \mathrm{mM}$ dGTP, $10 \mathrm{mM}$ dCTP, and10 mM dTTP), $10 \mu \mathrm{l}$ 10x error-prone-buffer (100 mM Tris- $\mathrm{HCl}$ (pH8), $500 \mathrm{mM} \mathrm{KCl}, 70 \mathrm{mM} \mathrm{MgCl}_{2}$, and $0.1 \%$ (W/V) Gelatine), $1.5 \mu \mathrm{l}$ $\mathrm{MnCl}_{2}$ (10mM stock solution), and $2 \mu \mathrm{l}$ Taq-Polymerase were mixed. Water was added to the mixture to a final volume of $50 \mu \mathrm{l}$. The mixture was separated into six PCR tubes. The PCR reaction was performed as shown in Table IV.3.

Table VI.3: The thermocycler protocol for error-prone mutagenesis

\begin{tabular}{|l|l|l|}
\hline temperature $\left[{ }^{\circ} \mathrm{C}\right]$ & time $[\mathrm{s}]$ & repetitions \\
\hline 94 & 60 & $1 \times$ \\
\hline 94 & 30 & \\
55 & 45 & $30 \times$ \\
72 & 60 & \\
\hline 72 & 120 & $1 \times$ \\
\hline
\end{tabular}

Afterwards the PCR reactions were pooled and subsequently digested by adding $1 \mu \mathrm{l} D p n /$ and incubation at $37^{\circ} \mathrm{C}$ over night. Finally, the PCR product, consisting out of a collection of mutated RSFP sequences, was cloned into the expression 
vector pQE31 (Qiagen, Hilden, Germany) and expressed in the E. coli strain BL21-CP-RIL.

\section{Screening strategies}

To screen for enhanced RSFP variants a laser based custom built screening setup was used, which was established in the reported work. The setup is described in detail in Chapter IV.1.

\section{The screening protocol}

The screening system searched for $E$. coli colonies. If a colony was found, it was automatically focused. Subsequently a defined illumination sequence was applied to the RSFP expressing E. coli cells. First, the proteins were switched off with $491 \mathrm{~nm}$ light $\left(\sim 8 \mathrm{KW} / \mathrm{cm}^{2}\right)$. Subsequently proteins were switched on by adding light of $405 \mathrm{~nm}\left(\sim 10 \mathrm{~kW} / \mathrm{cm}^{2}\right)$. This off-on-switching cycle was repeated four times. Finally, just $405 \mathrm{~nm}$ light $\left(\sim 10 \mathrm{~kW} / \mathrm{cm}^{2}\right)$ was applied to detect fluorescence excitation caused by this wavelength of light. The recorded data contained the key information to determine brightness, switching speeds, bleaching, and switching fatigue of the expressed protein variant. Subsequently the screening system moved on to the next colony until the whole plate was screened.

\section{Protein Purification and Characterisation}

\section{a. Protein Expression and Purification}

Proteins were expressed in the E. coli strain BL21-CP-RIL. For purification E. coli cells were harvested from agar plates using a cell scraper (Sarstedt, Newton, $\mathrm{NC}$ ) and resuspended in lysis buffer (10 mM Imidazole, $50 \mathrm{mM} \mathrm{NaH} \mathrm{PO}_{4}, 300$ $\mathrm{mM} \mathrm{NaCl}, \mathrm{pH}$ 8.0). After a treatment with lysozyme on ice for 2 hours a proteinase inhibitor was added (Complete, EDTA-free, Roche-Diagnostics, Mannheim, Germany). To lysate cells, five freeze-thaw cycles were performed using liquid nitrogen. Subsequently cell fragments were pelleted by centrifugation $\left(13,000 \mathrm{rpm}, 4{ }^{\circ} \mathrm{C}\right.$, Biofuge Fresco, Heraeus Hanau, Germany). The proteins of 
the supernatant were purified by Ni-NTA affinity chromatography (His SpinTrap, GE Healthcare, Freiburg, Germany) according to the manufacturer's instructions. The purified proteins were concentrated by ultrafiltration. Subsequently the elution buffer was exchanged against $100 \mathrm{mM}$ Tris- $\mathrm{HCl}, 150 \mathrm{mM} \mathrm{NaCl}, \mathrm{pH} 7.5$ by using gravity flow NAP5-columns (Sephadex G25-DNS-Grade, GE Healthcare, Freiburg, Germany). Proteins were stored at $4{ }^{\circ} \mathrm{C}$.

\section{b. Determination of Absorption Spectra}

For the determination of the absorption spectra of rsEGFP and rsEGFP2, a 100 $\mu$ l protein solution $(\mathrm{pH} 7.5)$ was analyzed with a photospectrometer (Varian Cary 4000 UVIVIS, Varian, Palo Alto, CA, USA) using a cuvette with a path length of 1 $\mathrm{cm}$. If necessary, purified proteins were diluted in the standard protein buffer (100 $\mathrm{mM}$ Tris $\mathrm{pH} 7.5,150 \mathrm{mM} \mathrm{NaCl})$.

Irradiation-dependent changes in the absorption were quantified by illuminating the protein solution in a cuvette with a fiber coupled mercury lamp (Leica, Wetzlar, Germany) equipped with a $(488 \pm 5) \mathrm{nm}$ excitation filter. For each measurement of the spectrum the irradiation was briefly interrupted.

To determine the absorption spectra at different $\mathrm{pH}$-values the protein samples were diluted 50-fold in the appropriate buffer $(\mathrm{pH} 4-6,0.1 \mathrm{M}$ citrate buffer; $\mathrm{pH} 7$ - 8, 0.1 M Tris- $\mathrm{HCl}$; and $\mathrm{pH}$ 9-10, 0.1 M glycine buffer).

\section{c. Determination of Fluorescence Excitation and Emission Spectra}

To measure excitation and emission spectra a fluorescence spectrometer (Varian Cary Eclipse, Varian, Palo Alto, CA, USA) and a cuvette with a path length of 1 $\mathrm{cm}$ were utilized. Excitation spectra were determined by measuring fluorescence at $540 \mathrm{~nm}$ (rsEGFP) and $520 \mathrm{~nm}$ (rsEGFP2). To determine emission spectra rsEGFP was excited at $450 \mathrm{~nm}$ and rsEGFP2 at $460 \mathrm{~nm}$. 


\section{d. Calculation of Molecular Extinction Coefficients and Fluorescence Quantum Yield of rsEGFP and rsEGFP2}

\section{Molecular Extinction Coefficient Determination}

The absorption spectra of rsEGFP, rsEGFP2 and EGFP were baseline corrected and normalized to the 280nm peak. Note, the number of the amino acids Trp, Phe, and Met were the same in all three proteins. Subsequently the molecular extinction Coefficients of rsEGFP and rsEGFP2 were estimated by a relative comparison of their absorption spectra and the spectrum of EGFP $(\varepsilon=53,000$ (Patterson, G.H. et al. 1997)).

\section{Quantum Yield Determination}

To determine the quantum yields of rsEGFP and rsEGFP2 the fluorescence emission spectra of rsEGFP, rsEGFP2 and EGFP were determined by illuminating at a defined excitation light wavelength. The proteins absorption values at the same wavelength where used to normalize the respective emission spectra. The integrals of the normalized emission spectra are values of the amount of fluorescence for each protein. The quantum yields of rsEGFP and rsEGFP2 were determined by a relative comparison of their integral values to the integral value of EGFP ( $\Phi_{\mathrm{FL}}=0.6$ (Patterson, G.H. et al. 1997))

\section{e. Estimation of Single Molecule Brightness}

The single-molecule brightness of rsEGFP, rsEGFP2, EGFP, and Dronpa were determined using fluorescence fluctuation spectroscopy, specifically fluorescence correlation spectroscopy (FCS)(Magde, D. et al. 1972; Ehrenberg, M. and Rigler, R. 1974; Haustein, E. and Schwille, P. 2003) and fluorescence intensity distribution analysis (Chen, Y. et al. 1999; Kask, P. et al. 1999). Both methods analyze characteristic fluctuations $\delta F(t)$ in the fluorescence signal $F(t)$ in time $t$ about an average value $F(t)=\langle F(t)\rangle+\delta F(t)$ by either calculating the secondorder auto-correlation function $\mathrm{G}\left(t_{c}\right)$ (FCS, with correlation time $t_{c}$ ) or by building up a frequency histogram $P(n, \Delta T)$ of photon counts detected per time window 
$\Delta T$ (FIDA, with number of photons $n$ ). Fluctuations in $F$ arise for example from diffusion of the FPs in and out of the confocal detection volume or by transitions into and out of a dark state such as the triplet, other metastable dark or the switch-off state.

FCS and FIDA data were analyzed using common theory (Widengren, J. et al. 1995; Eggeling, C. et al. 1998; Kask, P. et al. 1999). As outlined in detail previously (Eggeling, C. et al. 2007), the analysis most importantly resulted in two characteristic molecular parameters of the fluorescent proteins: the singlemolecule brightness (or count-rate per particle) $q$ and the observation time Tobs. Without saturating the excitation, the brightness $q \sim \Phi_{F L} \varepsilon$ scales with the fluorescence quantum yield $\Phi_{F L}$ and the extinction coefficient $\varepsilon$. For EGFP, the observation time Tobs is given by its average transit time through the focal spot, while for rsEGFP, rsEGFP2, and Dronpa it is given by both the transit time and if faster - the average switch-off time (Eggeling, C. et al. 2007).

The fluorescence fluctuation data were recorded at the same microscope setup as was used for RESOLFT imaging (see Chapter VI.6.b) using only the $491 \mathrm{~nm}$ laser line, a water immersion objective (60× UPLSAPO, NA 1.2, Olympus, Japan), two single-photon avalanche photodiodes (SPCM-AQR-13-FC, Perkin Elmer Optoelectronics, Fremont, CA) coupled to a 50:50 fiber splitter (FONT, Surrey, Canada) serving as the confocal pinhole ( 1.4 times the Airy disc of the image laser spot), and a hardware correlator (Flex02-01D, Correlator.com, NJ) for further processing of the fluorescence counts for FCS and FIDA ( $\Delta T=62.5$ $\mu s)$. Data were recorded for different powers of the $491 \mathrm{~nm}$ excitation laser and for the fluorescent proteins in the standard protein buffer $(100 \mathrm{mM}$ Tris- $\mathrm{HCl}, 150$ $\mathrm{mM} \mathrm{NaCl}, \mathrm{pH}$ 7.5).

\section{f. Determination of Switching and Bleaching Characteristics}

Switching curves of rsEGFP, rsEGFP2, or Dronpa were mainly recorded on PAA layers (except the measurements comparing switching speeds of rsEGFP and rsEGFP2 in Figure IV.29, which were performed on proteins fused to Vimentin and expressed in PtK2 cells). Switching was performed by alternate irradiation with light of $405 \mathrm{~nm}$ and $491 \mathrm{~nm}$ using a custom built CLSM equipped with a $100 \times 1.4$ NA oil immersion objective lens (Leica, Wetzlar, Germany). The used 
light intensities differed for the different experiments and are listed in connection with the experimental results in Chapter IV.

To measure switching fatigue the duration of the on/off switching cycles was adjusted individually for each protein by determining the minimum time to reach the maximum/minimum fluorescence.

Photo-bleaching of the on state was measured on proteins immobilized in a PAA layer by constant irradiation with $405 \mathrm{~nm}\left(1 \mathrm{~kW} / \mathrm{cm}^{2}\right)$ and $491 \mathrm{~nm}\left(3 \mathrm{~kW} / \mathrm{cm}^{2}\right)$ light.

\section{g. Determination of Relaxation Kinetics}

To measure the thermal relaxation from the off state into the fluorescent equilibrium state, rsEGFP was embedded in a PAA layer (see Chapter VI.5.a). Using a custom built confocal scanning microscope, the rsEGFP was switched on with $405 \mathrm{~nm}$ light in an area of $12 \times 12 \mu \mathrm{m}^{2}$ and subsequently the central $6 \times 6$ $\mu^{2}$ were turned off with $491 \mathrm{~nm}$ light. The level of thermal relaxation from the off state into the fluorescent equilibrium state was determined by irradiation with light of $491 \mathrm{~nm}$ after a defined time. For each data point the experiment was repeated. The ratio of the average intensity in the central square (initially off) versus the average intensity of the surrounding frame (always on) represents the fraction of thermally relaxed rsEGFP. The intensity and time of the $491 \mathrm{~nm}$ light for probing of the thermally relaxed fluorescence was kept very low to avoid switching-off during this period and thus to avoid an overestimation of the thermal relaxation time.

\section{h. Semi-Native Gel Electrophoresis}

Semi-native polyacrylamide gel electrophoresis was used to determine the oligomeric state of the different FPs. $20 \mu \mathrm{g}$ of each protein were suspended in a concentrated sucrose solution to a final concentration of $10 \%(\mathrm{w} / \mathrm{v})$ sucrose. As size markers monomeric EGFP, dimeric dTomato, and tetrameric DsRed were used. The samples were loaded on a two-phase polyacrylamide gel consisting out of a $12.5 \%$ separation gel $(6.3 \mathrm{ml} \mathrm{H} 2 \mathrm{O}$ MilliQ, $5 \mathrm{ml} 1.5 \mathrm{M}$ Tris- $\mathrm{HCl} \mathrm{pH} 8.8$, $8.3 \mathrm{ml}$ Rotiphorese Gel 30 solution (Roth, Karlsruhe, Germany), $200 \mu \mathrm{l} 10 \%$ 
(w/v) sodiumdodecyl sulphate (SDS), $200 \mu \mathrm{l} 10 \%(\mathrm{w} / \mathrm{v})$ ammonium persulfate (APS), $20 \mu \mathrm{l}$ Tetramethylethylendiamin (TEMED)) and a $5 \%$ loading gel $(5.6 \mathrm{ml}$ $\mathrm{H}_{2} \mathrm{O}$ MilliQ, 2.5ml 1.5 M Tris-HCl pH 6.8, $1.7 \mathrm{ml}$ Rotiphorese Gel 30 solution, 100 $\mu \mathrm{l} 10 \%(\mathrm{w} / \mathrm{v})$ SDS, $100 \mu \mathrm{l} 10 \%$ (w/v) APS, $10 \mu \mathrm{l} \mathrm{TEMED).} \mathrm{Electrophoresis} \mathrm{was}$ performed at $20 \mathrm{~mA}$.

The different protein bands were detected using a custom built fluorescence gel reader. The green fluorescence of EGFP, rsEGFP, and rsEGFP2 was detected at $525 \pm 30 \mathrm{~nm}$ when excited with light of $470 \pm 5 \mathrm{~nm}$. The red fluorescence of dTomato and DsRed was excited at $545 \pm 10 \mathrm{~nm}$ and detected at $617 \pm 37 \mathrm{~nm}$.

\section{i. Size Separation Chromatography}

Size separation chromatography was performed with a Superdex 200 column (Pharmacia, Uppsala, Schweden) and an Äkta prime plus system (GE Healthcare, Freiburg, Germany). Prior to chromatography, proteins were concentrated to $3 \mathrm{mg} / \mathrm{ml}$ (100 mM Tris- $\mathrm{HCl}, 150 \mathrm{mM} \mathrm{NaCl}, \mathrm{pH}$ 7.5). The flow rat was set to $4 \mathrm{ml} / \mathrm{min}$. Protein absorption was monitored at $280 \mathrm{~nm}$. All measurements were performed at $4{ }^{\circ} \mathrm{C}$.

\section{j. Determination of Chromophor Maturation Halftime}

To determine the chromophore maturation time of rsEGFP/rsEGFP2, the E. coli cell strain TOP10 (Invitrogen) was transformed with the pBad-rsEGFP / pBadrsEGFP2 plasmid and grown overnight at $37{ }^{\circ} \mathrm{C}$ in LB-Amp medium. The overnight culture was used to inoculate $200 \mathrm{ml}$ LB-Amp growth medium and grown to an OD600 of $0.5-0.6$ at $37{ }^{\circ} \mathrm{C}$. The addition of arabinose to a final concentration of $0.2 \%$ induced the protein expression. The cultures were further incubated at $37^{\circ} \mathrm{C}$ for 4 hours (rsEGFP) or 2 hours (rsEGFP2). Finally, cells were opened up and pelleted by centrifugation. rsEGFP/rsEGFP2 was purified immediately from the supernatant using a His SpinTrap column (GE Healthcare, Freiburg, Germany). The proteins were diluted in buffer (final concentration: 20 $\mathrm{mM} \mathrm{NaH} 2 \mathrm{PO} 4,500 \mathrm{mM} \mathrm{NaCl}, 30 \mathrm{mM}$ imidazol, pH 7.5). Care was taken that all preparation steps took place at $4{ }^{\circ} \mathrm{C}$. Finally, fluorescence emission spectra of 
rsEGFP/rsEGFP2 were taken at several time points using a fluorescence spectrometer (Varian Cary Eclipse) while incubating the protein solution at $37^{\circ} \mathrm{C}$.

\section{Sample preparation}

\section{a. Preparation of Protein Polyacrylamide Layers}

$24.5 \mu \mathrm{l}$ purified protein ( 0.09 $\mathrm{mM})$ was mixed with $17.5 \mu \mathrm{l}$ Tris- $\mathrm{HCl} \mathrm{pH} \mathrm{7.5,} 30 \mu \mathrm{l}$ acrylamide (Rotiphorese Gel 30, Roth, Karlsruhe, Germany), $0.75 \mu \mathrm{l}$ 10\% APS and $1 \mu \mathrm{l} 10 \%$ TEMED. Immediately after mixing $\sim 10 \mu \mathrm{l}$ of this solution was placed on a glass slide and a cover slip was pressed onto the sample to ensure an appropriate thin layer. After the sample was completely polymerized, it was sealed with silicon-based glue (Picodent twinsil, Picodent, Wipperfürth, Germany) to prevent drying-out.

\section{b. Preparing E. coli samples Expressing the rsEGFP-MreB}

\section{Fusion Protein}

E. coli cells of strain SURE or MC1000 were transformed with the plasmid prsEGFP-MreB and grown on LB agar plates containing ampicillin over night at $37^{\circ} \mathrm{C}$. A single colony was picked and transferred to $5 \mathrm{ml}$ LB-Amp medium. Cells were grown over night at $30^{\circ} \mathrm{C}$ on a shaker.Subsequently, the cell suspension was again diluted and the cells were gown to an OD600 of $\sim 0.6$ at $30{ }^{\circ} \mathrm{C}$. Protein expression was induced by adding $20 \mu \mathrm{M}-100 \mu \mathrm{M}$ Isopropyl- $\beta-D-$ thiogalactopyranosid (IPTG). After induction, the cells were grown at $25^{\circ} \mathrm{C}$ or 30 ${ }^{\circ} \mathrm{C}$ under agitation for 1-2 days.

To prepare the samples for microscopic measurements the $E$. coli cells were immobilized on agar coated microscopy slides (Pfennig, N. and Wagener, S. 1986).

For slide preparation powdered agar was washed twice in water and taken up to $2 \% \mathrm{w} / \mathrm{v}$ in water. The suspension was heated to dissolve the agar. Subsequently $1 \mathrm{ml}$ of the agar solution was spread uniformly on a clean microscope slide and dried over night. 
$10 \mu$ of a prepared $E$. coli sample was placed onto one coated slide, covered with a cover slip and sealed with silicone-based glue (Picodent twinsil, Picodent, Wipperfürth, Germany) to prevent dring-out.

\section{c. Preparing Mammalian Cell Samples for Microscopy}

PtK2 cells were grown and transfected on cover slips placed in microtiter plates (see Chapter VI.1.b). To prepare the cells for microscopic measurements, 24 - 72 $\mathrm{h}$ after transfection the cover slips were alternatively placed on a microscopy slide and sealed with silicone-based glue (Picodent twinsil, Picodent, Wipperfürth, Germany) or transferred into a custom built live cell chamber and covered with fresh DMEM growth medium of $37^{\circ} \mathrm{C}$.

\section{d. Preparation of Organotypic Hippocampal Slice Cultures for Micorscopy}

16 -48 hours after viral transfection slice cultures were transferred to an imaging chamber at room temperature and sustained in artificial cerebrospinal fluid, containing $126 \mathrm{mM} \mathrm{NaCl}, 2.5 \mathrm{mM} \mathrm{KCl}, 2.5 \mathrm{CaCl}_{2}, 1.3 \mathrm{mM} \mathrm{MgCl}$, $30 \mathrm{mM}$ glucose and $27 \mathrm{mM}$ HEPES; the $\mathrm{pH}$ was adjusted with $\mathrm{NaOH}$ to 7.4 .

\section{Fluorescence Microscopy}

\section{a. Confocal Laser Scanning Microscopy}

Conventional CLSM was performed on the one hand to determine the localization pattern of various fusion proteins (Figure IV.9 and IV.28) and on the other hand to demonstrate switching of the Keratin19-rsEGFP fusion in living PtK2 cells (Figure IV.10). Imaging was performed using the TCS SP5 CLSM (Leica Microsystems, Wetzlar, Germany). Images were recorded using a UV-corrected 1.4 NA oil immersion objective lens (63× HCX PL APO, Leica, Wetzlar, Germany) and a pinhole diameter of one Airy unit. To image the rsEGFP variants the sample was illuminated with $488 \mathrm{~nm}$ and $405 \mathrm{~nm}$ laser light simultaneously. To switch the sample into the off-state it was scanned only with $488 \mathrm{~nm}$ light until the sample reached a fluorescence minimum. To switch the sample back on, the 
sample was scanned with both wavelengths of light until the sample reached a fluorescence maximum.

\section{b. RESOLFT Microscopy}

For RESOLFT imaging a custom built microscope setup (built by Marcel Leutenegger, Ilaria Testa, and Hannes Bock) was used (Figure VI.2), which provides one $405 \mathrm{~nm}$ focus (laser source: $30 \mathrm{~mW}$, BLC-030-405-S, CrystaLaser, Reno, NV) and two alternative $491 \mathrm{~nm}$ foci (laser source: $50 \mathrm{~mW}$ Calypso 50, Cobolt, Stockholm, Schweden): (i) a normal diffraction-limited focus for reading out the fluorescence of rsEGFP/rsEGFP2 and (ii) a focus with a central intensity minimum ("zero") for switching proteins at the focal periphery off.

To optimize the beam profile of the $405 \mathrm{~nm}$ beam it was coupled through an optical fiber. The collimation lens for coupling out the $405 \mathrm{~nm}$ beam was an achromatic doublet with $100 \mathrm{~mm}$ focal length to fill the objective aperture. The linear polarization of the $405 \mathrm{~nm}$ beam was roughly circularized by a quarter wave plate.

To obtain the two alternatively switchable $491 \mathrm{~nm}$ foci the following setup was inserted into the $491 \mathrm{~nm}$ laser beam. A half wave plate allowed adjusting the polarization of the $491 \mathrm{~nm}$ laser beam to match the axis of a Glan-Thompson polarizer (B. Halle). An electro-optic modulator (LM 0202 5W 400-650nm with pulse amplifier LIV 20, Linos) rotated the linear polarization by $90^{\circ}$ for alternating between horizontal and vertical polarization. The vertically polarized beam was reflected by a polarizing beam splitter (PTW5 450-650nm, B. Halle) and spatially filtered by a Kepler telescope consisting of a $40 \mathrm{~mm}$ achromatic doublet, a $10 \mu \mathrm{m}$ pinhole (Linos) in the common focal plane, and a $100 \mathrm{~mm}$ achromatic doublet. The horizontally polarized beam went straight through the polarizing beam splitter and was sent through an identical spatial filter. It then passed through a $2 \pi$ phase ramp (463 nm mask, vortex plate VPP-A, RPC Photonics, Rochester, NY) for creating a lateral doughnut. A second polarizing beam splitter (PWT5) recombined both beams thereafter. Alternating the polarization allowed selecting either of the two $491 \mathrm{~nm}$ beam paths with a mutual contrast better than 2000:1. Finally, a quarter wave and a half wave plate (B. Halle) were introduced in front of the objective for circularizing the polarization of the $491 \mathrm{~nm}$ doughnut beam. 
Hence, two independent spatial filters were set up mainly for minimizing the wave front aberrations, in particular those introduced by the polarizing beam splitters, as well as for independently adjusting the depths of the switching and the reading foci.

For the mutual alignment of the beams, a pellicle ( $8 \%$ reflectivity, BP 108 , Thorlabs) was flipped in between the main dichroic mirror and the wave plates, which prevented any change of the polarization of the $491 \mathrm{~nm}$ beam.

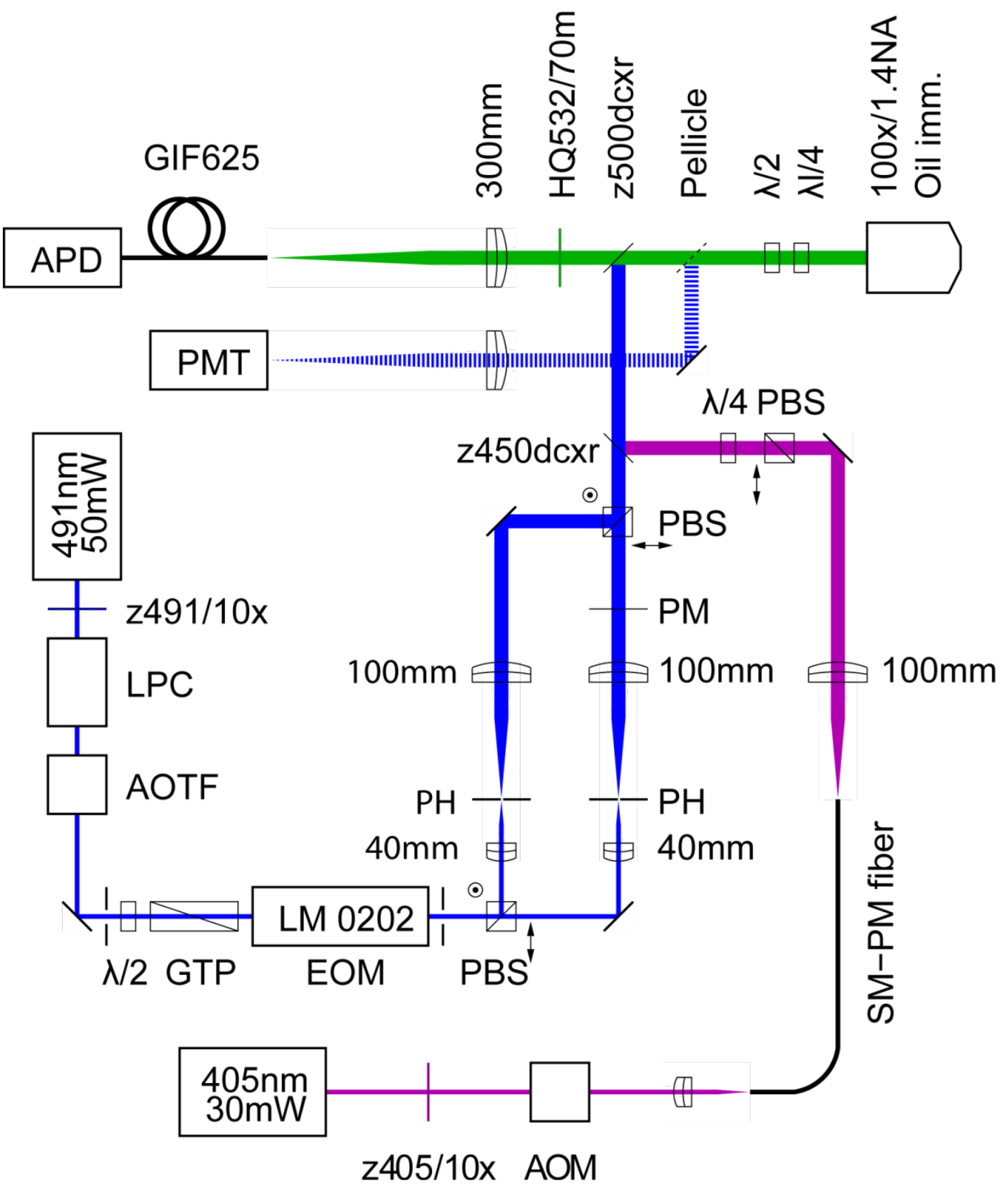

Figure VI.2: Scheme of the established RESOLFT setup. LPC: laser power controller; AOTF AOM: acousto-optic modulators; GTP: Glan-Thompson polarizer; EOM: electro-optic modulator (polarization rotator); PBS: polarizing beam splitter; PH: pinhole (10 $\mu \mathrm{m}$ in diameter); PM: phase mask (vortex plate or phase disc). 
To search for live cells expressing rsEGFP fusion proteins, a lens was inserted ahead of the main dichroic mirror, which resulted in a wide-field illumination of an area of 30-40 $\mu \mathrm{m}$ in diameter. The sample could be visualized by using an ocular in a separate beam path that was addressable instead of the confocal pinhole. To finally record RESOLFT images a fiber coupled APD was utilized to detect the fluorescence signal of each scanning position.

To obtain RESOLFT images the following illumination sequence was performed at each scanning position:

1) The proteins were switched into the on-state by irradiation with $405 \mathrm{~nm}$ light.

2) In case of imaging rsEGFP a 2 ms lasting illumination break was performed to let activated proteins equilibrate.

3) The proteins at the focal periphery were switched into the non-fluorescent state by applying a doughnut shaped beam of $491 \mathrm{~nm}$.

4) The remaining on-state proteins at the focal center were probed by light of $491 \mathrm{~nm}$.

The light intensities and the illumination times for each sequence step differed depending on the performed experiment and the used rsEGFP variant. They are listed for each experiment in Chapter IV.

rsEGFP-samples were scanned in a hope-mode. The following scanning position was targeted further away from the previous position than the size of the focal spot. Thus the sample was scanned in several passes by recording only every $N$ th pixel per pass. $N$ was chosen such that rsEGFP could equilibrate into the bright state before it underwent another on/off cycle.

rsEGFP2-samples were scanned in the classical linear way.

\section{c. Image Deconvolution}

Deconvolution of images was performed using the Richardson Lucy algorithm (Richardson, W.H. 1972; Lucy, L.B. 1974) to subtract residual fluorescence in the periphery of the focal spot. 10 iterations were performed assuming an effective 
PSF, which consists out of $30 \%$ diffraction limited PSF (FWHM $200 \mathrm{~nm}$ ) and 70 $\%$ RESOLFT PSF (50 nm FWHM).

\section{Data Storage Approaches}

\section{a. Rewritable Data Storage}

A custom-built CLSM (built by Marcel Leutenegger) was used for writing and reading. The $491 \mathrm{~nm}$ excitation light was provided by a solid-state laser (50 $\mathrm{mW}$, Calypso 50, Cobolt, Stockholm, Sweden), whose output power was reduced by a laser power controller (LPC-VIS, Brockton Electro-Optics, Brockton, MA) and modulated by an acousto-optic modulator (AOTF.nC/TN, AA Opto-Electronic, Orsay, Cedex, France). The $405 \mathrm{~nm}$ UV light was generated by a diode pumped crystal laser (30 mW, BCL-030-405-S, CrystaLaser, Reno, NV). Its power was reduced by a neutral density filter and modulated by an acousto-optic modulator (MTS 130A3, AA Opto-Electronic). Shutters blocked the beams during long nonoperational periods of time. Each laser beam was cleaned up by a laser line clean-up filter (z491/10x and z405/10x, AHF Analysentechnik, Tübingen, Germany). The coupled out beams were collimated with achromatic lenses of 50 $\mathrm{mm}$ focal length (Linos Photonics, Göttingen, Germany). A dichroic mirror (z450dcxr, AHF) combined the laser beams and a dichroic mirror (z500dcxr, $\mathrm{AHF}$ ) reflected them into the aperture of the $100 \times 1.4 \mathrm{NA}$ oil immersion objective (HCX PC APO 100×/1.40-0.70 oil, Leica Microsystems, Wetzlar, Germany). A three-axis piezo stage (NanoMAX311 with BPC203, Thorlabs, München, Germany) was used to position and scan the sample. The induced fluorescence from the excited focal region was focused by an achromatic tube lens of $300 \mathrm{~mm}$ focal length (Linos) onto a gradient-index multi-mode fiber with $62.5 \mu \mathrm{m}$ core diameter (GIF625, Thorlabs) acting as confocal pinhole. A band pass filter (HQ532/70m, AHF) rejected back-reflected and/or stray laser light. An avalanche photo-diode (APD) (SPCM-AQR-13-FC, PerkinElmer, Salem, MA) detected the fluorescence photons, which were finally counted and recorded on a personal computer. A data acquisition card (NI-DAQ PCI-6731, National Instruments, Austin, TX) was used to generate the scan voltages and the AOM drive voltages as well as the blanking and synchronization signals. Custom MATLAB (The 
MathWorks, Natick, MA) programs allowed automated generation of the voltages and signals for moving the sample to the desired coordinates and for generating the desired laser pulses, as well as for recording the fluorescence images. The laser beams were mutually aligned by recording the laser light back-scattered by a silver nanobead (60 nm silver colloid, BBInternational, Cardiff, UK) with a photo-multiplier tube (PerkinElmer). Images were taken using the software Imspector (www.imspector.de).

The text data of the book "Grimm`s Fairy Stories" (http://www.gutenberg.org /files/11027/11027.txt) were sequentially written into a rsEGFP-PAA-layer and read out as described in Chapter IV.2.c.

\section{b. Diffraction Unlimited All-Optical Data Storage}

For diffraction unlimited all-optical writing and reading a further beam path of 532 $\mathrm{nm}$ light was added to the RESOLFT setup (Chapter VI.6.b). Single spots were written by specific bleaching of on-state proteins in the center of the focus as described in Chapter IV.3.e. The written spot patterns were read out by RESOLFT imaging (1. on-switching: $405 \mathrm{~nm}, \sim 1 \mathrm{~kW} / \mathrm{cm}^{2}, 100 \mu \mathrm{s} / 2$. illumination break: $2 \mathrm{~ms} / 3$. off-switching in the focal periphery using a doughnut shaped beam: $491 \mathrm{~nm}, \sim 1 \mathrm{~kW} / \mathrm{cm}^{2}, 10-20 \mathrm{~ms} /$ fluorescent read out of on-state protein in the focal center: $491 \mathrm{~nm}, \sim 1 \mathrm{~kW} / \mathrm{cm}^{2}, 1-2 \mathrm{~ms}$ ). 


\section{Abbreviations}

\begin{tabular}{|c|c|}
\hline AFM & Atomic Force Microscopy \\
\hline amFP486 & Anemonia majano Fluorescent Protein 486 \\
\hline AOM & Acousto-Optic Modulator \\
\hline AOTF & Acousto-Optic Transmission Filter \\
\hline APD & Avalanche PhotoDiode \\
\hline APS & Ammonium PerSulfate \\
\hline ASCII & American Standard Code for Information Interchange \\
\hline asFP595 & Anemonia sulcata Fluorescent Protein 595 \\
\hline avGFP & Aequoria victoria Green Fluorescent Protein \\
\hline BFP & Blue Fluorescent Protein \\
\hline bp & base pair \\
\hline CCD & Calcite Compensation Depth \\
\hline cDNA & coding Desoxyribonucleidacid \\
\hline CFP & Cyan Fluorescent Protein \\
\hline CLSM & Confocal Laser Scanning Microscopy \\
\hline C-terminus & Carboxy-terminus \\
\hline DNA & Desoxyribonucleicacid \\
\hline DIC & Differential Interference Contrast \\
\hline DMEM & Dulbecco's Modified Eagle's Medium \\
\hline dNTPs & Deoxynucleoside triphosphate \\
\hline dsRed & Discosoma sp.red fluorescent protein \\
\hline E. coli & Escherichia coli \\
\hline EBFP & Enhanced Blue Fluorescent Protein \\
\hline ECFP & Enhanced Cyan Fluorescent Protein \\
\hline EFMPlus & Eight-to-Fourteen-Modulation-plus \\
\hline EGFP & Enhanced Green Fluorescent Proteins \\
\hline eqFP578 & Entacmaea quadricolor Fluorescent Protein 578 \\
\hline eqFP611 & Entacmaea quadricolor Fluorescent Protein 611 \\
\hline ER & Endoplasmic reticulum \\
\hline ESPT & Excited State Proton Transfer \\
\hline EYFP & Enhanced Yellow Fluorescent Protein \\
\hline FACS & Fluorescence Activated Cell Sorter \\
\hline FBS & Fetal Bovine Serum \\
\hline FCS & Fluorescence Correlation Spectroscopy \\
\hline FIDA & Fluorescence Intensity Distribution Analysis \\
\hline FP & Fluorescent Protein \\
\hline FRET & Förster Resonance Energy Transfer \\
\hline FWHM & Full Width Half Maximum \\
\hline GFP & Green Fluorescent Protein \\
\hline GSDIM & Ground State Depletion Microscopy \\
\hline HEPES & N-2-hydroxyethyl-piperazin-N-2-ethansulfonic acid \\
\hline IPTG & Isopropyl- $\beta-D$-thiogalactopyranosid \\
\hline kDA & kilo Dalton \\
\hline KFP1 & Kindling Fluorescent Protein \\
\hline LB medium & Luria Bertani medium \\
\hline mEGFP & Monomeric Enhanced Green Fluorescent Protein \\
\hline mOrange & monomeric Orange Fluorescent Protein \\
\hline
\end{tabular}




$\begin{array}{ll}\text { mRFP1 } & \text { monomeric Red Fluorescent Protein 1 } \\ \text { N-terminus } & \text { Amino-terminus } \\ \text { NA } & \text { Numerical Aperture } \\ \text { OD } & \text { Optical Density } \\ \text { PAA } & \text { Polyacrylamid } \\ \text { PA-GFP } & \text { weitere S.27 } \\ \text { PALM } & \text { Photoactivation Localization Microscopy } \\ \text { PALMIRA } & \text { Photoactivated localization microscopy with independently } \\ & \text { running acquisition } \\ \text { PCR } & \text { Polymerase Chain Reaction } \\ \text { PMT } & \text { PhotoMultiplier Tube } \\ \text { PSF } & \text { Point Spread Function } \\ \text { RESOLFT } & \text { Reversible Saturable Optical Fluorescence Transition } \\ \text { ROS } & \text { reactive oxygen species } \\ \text { rSEGFP } & \text { reversibly switchable Enhanced Green Fluorescent } \\ & \text { Proteins } \\ \text { RSFP } & \text { Reversibly Switchable Fluorescent Protein } \\ \text { SDS } & \text { Sodiumdodecyl Sulphate } \\ \text { SMS } & \text { Single Molecule Switching } \\ \text { sp. } & \text { species } \\ \text { STED } & \text { Stimulated Emission Dipletion } \\ \text { STM } & \text { Scanning Tunneling Microscopy } \\ \text { STORM } & \text { Stochastic Optical Reconstruction Microscopy } \\ \text { tdTomato } & \text { tandem dimer Tomato } \\ \text { TEM } & \text { Transmission Electron Microscopy } \\ \text { TEMED } & \text { Tetramethylethylendiamin } \\ \text { UV } & \text { ultra violett } \\ \text { YFP } & \text { Yellow Fluorescent Protein } \\ & \end{array}$




\section{References}

Abbe, E. (1873). "Beiträge zur Theorie des Mikroskops und der mikroskopischen Wahrnehmung." Archiv für Mikroskopische Anatomie 9: 413-468.

Abbe, E. (1880). "Üleber die Grenzen der geometrischen Optik." Jenaische Zeitschrift fuer Naturwissenschaft, Sitzungsberichte 14: 71 - 109.

Abbyad, P., Childs, W., Shi, X. and Boxer, S.G. (2007). "Dynamic Stokes shift in green fluorescent protein variants." Proc Natl Acad Sci USA 104(51): 20189-20194.

Adam, V., Lelimousin, M., Boehme, S., Desfonds, G., Nienhaus, K., Field, M.J., Wiedenmann, J., McSweeney, S., Nienhaus, G.U. and Bourgeois, D. (2008). "Structural characterization of IrisFP, an optical highlighter undergoing multiple photo-induced transformations." Proc Natl Acad Sci USA 105(47): 18343-18348.

Adam, V., Mizuno, H., Grichine, A., Hotta, J., Yamagata, Y., Moeyaert, B., Nienhaus, G., Miyawaki, A., Bourgeois, D. and Hofkens, J. (2010). "Data storage based on photochromic and photoconvertible fluorescent proteins." J Biotechnol 149(4): 289-298.

Aglyamova, G.V., Hunt, M.E., Modi, C.K. and Matz, M.V. (2011). "Multi-colored homologs of the green fluorescent protein from hydromedusa Obelia sp." Photochem Photobiol Sci. 10(8):1303-9

Ai, H.W., Henderson, J.N., Remington, S.J. and Campbell, R.E. (2006). "Directed evolution of a monomeric, bright and photostable version of Clavularia cyan fluorescent protein: structural characterization and applications in fluorescence imaging." Biochem J 400(3): 531-540.

Ai, H.W., Shaner, N.C., Cheng, Z., Tsien, R.Y. and Campbell, R.E. (2007). "Exploration of new chromophore structures leads to the identification of improved blue fluorescent proteins." Biochemistry 46(20): 5904-5910.

Alieva, N.O., Konzen, K.A., Field, S.F., Meleshkevitch, E.A., Hunt, M.E., BeltranRamirez, V., Miller, D.J., Wiedenmann, J., Salih, A. and Matz, M.V. (2008). "Diversity and evolution of coral fluorescent proteins." PLoS One 3(7): e2680.

Ando, R., Hama, H., Yamamoto-Hino, M., Mizuno, H. and Miyawaki, A. (2002). "An optical marker based on the UV-induced green-to-red photoconversion of a fluorescent protein." Proc Natl Acad Sci USA 99(20): $12651-12656$.

Ando, R., Mizuno, H. and Miyawaki, A. (2004). "Regulated Fast Nucleocytoplasmic Shuttling Observed by Reversible Protein Highlighting." Science 306(5700): 1370-1373.

Andresen, M., Stiel, A.C., Folling, J., Wenzel, D., Schonle, A., Egner, A., Eggeling, C., Hell, S.W. and Jakobs, S. (2008). "Photoswitchable fluorescent proteins enable monochromatic multilabel imaging and dual color fluorescence nanoscopy." Nat Biotechnol 26(9): 1035-1040.

Andresen, M., Stiel, A.C., Trowitzsch, S., Weber, G., Eggeling, C., Wahl, M.C., Hell, S.W. and Jakobs, S. (2007). "Structural basis for reversible photoswitching in Dronpa." Proc Natl Acad Sci USA 104: 13005-13009. 
Andresen, M., Wahl, M.C., Stiel, A.C., Gräter, F., Schäfer, L.V., Trowitzsch, S., Weber, G., Eggeling, C., Grubmüller, H., Hell, S.W. and Jakobs, S. (2005). "Structure and mechanism of the reversible photoswitch of a fluorescent protein." Proc Natl Acad Sci USA 102(37): 13070-13074.

Avwioro, G. (2011). "HISTOCHEMICAL USES OF HAEMATOXYLIN - A REVIEW." International Journal of Research and Reviews in Applied Sciences 1: 24 - 34.

Barer, R. (1952). "Combined phase-contrast and interference-contrast microscopy." Nature 169(4290): 108-109.

Barondeau, D.P., Kassmann, C.J., Tainer, J.A. and Getzoff, E.D. (2005). "Understanding GFP chromophore biosynthesis: controlling backbone cyclization and modifying post-translational chemistry." Biochemistry 44(6): 1960-1970.

Barondeau, D.P., Kassmann, C.J., Tainer, J.A. and Getzoff, E.D. (2007). "The case of the missing ring: radical cleavage of a carbon-carbon bond and implications for GFP chromophore biosynthesis." $\underline{\mathrm{J} \text { Am Chem Soc }}$ 129(11): 3118-3126.

Barondeau, D.P., Putnam, C.D., Kassmann, C.J., Tainer, J.A. and Getzoff, E.D. (2003). "Mechanism and energetics of green fluorescent protein chromophore synthesis revealed by trapped intermediate structures." Proc Natl Acad Sci USA 100(21): 12111-12116.

Berkovic, G., Krongauz, V. and Weiss, V. (2000). "Spiropyrans and Spirooxazines for Memories and Switches." Chemistry Reviews 100(5): 1741-1754.

Berning, S., Willig, K.I., Steffens, H., Dibaj, P. and Hell, S.W. (2012). "Nanoscopy in a living mouse brain." Science 335(6068): 551.

Betzig, E., Patterson, G.H., Sougrat, R., Lindwasser, O.W., Olenych, S., Bonifacino, J.S., Davidson, M.W., Lippincott-Schwartz, J. and Hess, H.F. (2006). "Imaging Intracellular Fluorescent Proteins at Nanometer Resolution." Science 313(5793): 1642-1645.

Bevis, B.J. and Glick, B.S. (2002). "Rapidly maturing variants of the Discosoma red fluorescent protein (DsRed)." Nat Biotechnol 20(1): 83-87.

Bingen, P., Reuss, M., Engelhardt, J. and Hell, S.W. (2011). "Parallelized STED fluorescence nanoscopy." Opt Express 19(24): 23716-23726.

Binnig, G., Quate, C.F. and Gerber, C. (1986). "Atomic force microscope." Phys Rev Lett 56: 930-933.

Binning, G. and Rohrer, H. (1982). "Scanning tunneling microscopy." Helvetica Physica Acta 55: 726-735.

Birnboim, H.C. and Doly, J. (1979). "A rapid alkaline extraction procedure for screening recombinant plasmid DNA." Nucleic Acids Res 7(6): 1513-1523.

Bizzarri, R., Serresi, M., Cardarelli, F., Abbruzzetti, S., Campanini, B., Viappiani, C. and Beltram, F. (2010). "Single amino acid replacement makes Aequorea victoria fluorescent proteins reversibly photoswitchable." $\underline{\mathrm{J} \mathrm{Am}}$ Chem Soc 132(1): 85-95.

Brakemann, T., Stiel, A.C., Weber, G., Andresen, M., Testa, I., Grotjohann, T., Leutenegger, M., Plessmann, U., Urlaub, H., Eggeling, C., Wahl, M.C., Hell, S.W. and Jakobs, S. (2011). "A reversibly photoswitchable GFP-like protein with fluorescence excitation decoupled from switching." Nat Biotechnol 29(10): 942-947. 
Brakemann, T., Weber, G., Andresen, M., Groenhof, G., Stiel, A.C., Trowitzsch, S., Eggeling, C., Grubmueller, H., Hell, S.W., Wahl, M.C. and Jakobs, S. (2010). "Molecular Basis of the Light-driven Switching of the Photochromic Fluorescent Protein Padron." J Biol Chemistry. 285(19): 14603-14609

Brejc, K., Sixma, T.K., Kitts, P.A., Kain, S.R., Tsien, R.Y., Ormo, M. and Remington, S.J. (1997). "Structural basis for dual excitation and photoisomerization of the Aequorea victoria green fluorescent protein." Proc Natl Acad Sci USA 94(6): 2306-2311.

Campbell, R.E., Tour, O., Palmer, A.E., Steinbach, P.A., Baird, G.S., Zacharias, D.A. and Tsien, R.Y. (2002). "A monomeric red fluorescent protein." Proc Natl Acad Sci USA 99(12): 7877-7882.

Casadaban, M.J. and Cohen, S.N. (1980). "Analysis of gene control signals by DNA fusion and cloning in Escherichia coli." J Mol Biol 138(2): 179-207.

Chalfie, M., Tu, Y., Euskirchen, G., Ward, W.W. and Prasher, D.C. (1994). "Green fluorescent protein as a marker for gene expression." Science 263(5148): 802-805.

Chattoraj, M., King, B.A., Bublitz, G.U. and Boxer, S.G. (1996). "Ultra-fast excited state dynamics in green fluorescent protein: Multiple states and proton transfer." Proc Natl Acad Sci USA 93(16): 8362-8367.

Chen, Y., Muller, J.D., So, P.T.C. and Gratton, E. (1999). "The photon counting histogram in fluorescence fluctuation spectroscopy." Biophys J 77: 553567.

Chudakov, D.M., Belousov, V.V., Zaraisky, A.G., Novoselov, V.V., Staroverov, D.B., Zorov, D.B., Lukyanov, S. and Lukyanov, K.A. (2003). "Kindling fluorescent proteins for precise in vivo photolabeling." Nat Biotechnol 21(2): 191-194.

Chudakov, D.M., Chepurnykh, T.V., Belousov, V.V., Lukyanov, S. and Lukyanov, K.A. (2006). "Fast and precise protein tracking using repeated reversible photoactivation." Traffic 7(10): 1304-1310.

Chudakov, D.M., Matz, M.V., Lukyanov, S. and Lukyanov, K.A. (2010). "Fluorescent proteins and their applications in imaging living cells and tissues." Physiol Rev 90(3): 1103-1163.

Chudakov, D.M., Verkhusha, V.V., Staroverov, D.B., Souslova, E.A., Lukyanov, S. and Lukyanov, K.A. (2004). "Photoswitchable cyan fluorescent protein for protein tracking." Nat Biotechnol 22(11): 1435-1439.

Cordes, T., Maiser, A., Steinhauer, C., Schermelleh, L. and Tinnefeld, P. (2011). "Mechanisms and advancement of antifading agents for fluorescence microscopy and single-molecule spectroscopy." Phys Chem Chem Phys 13(14): 6699-6709.

Cormack, B.P., Valdivia, R.H. and Falkow, S. (1996). "Facs-optimized mutants of the green fluorescent protein (Gfp)." Gene 173(1): 33-38.

Corredor, C.C., Huang, Z.-L. and Belfield, K.D. (2006). "Two-Photon 3D Optical Data Storage via Fluorescence Modulation of an Efficient Fluorene Dye by a Photochromic Diarylethene." Adv Mater 18: 2910-2914.

Craggs, T.D. (2009). "Green fluorescent protein: structure, folding and chromophore maturation." Chem Soc Rev 38(10): 2865-2875.

Dayel, M.J., Hom, E.F. and Verkman, A.S. (1999). "Diffusion of green fluorescent protein in the aqueous-phase lumen of endoplasmic reticulum." Biophys $\mathrm{J}$ 76(5): 2843-2851. 
Dedecker, P., Hotta, J.I., Flors, C., Sliwa, M., Uji, I.H., Roeffaers, M.B., Ando, R., Mizuno, H., Miyawaki, A. and Hofkens, J. (2007). "Subdiffraction Imaging through the Selective Donut-Mode Depletion of Thermally Stable Photoswitchable Fluorophores: Numerical Analysis and Application to the Fluorescent Protein Dronpa." J Am Chem Soc 129(51): 16132-16141.

Deheyn, D.D., Kubokawa, K., McCarthy, J.K., Murakami, A., Porrachia, M., Rouse, G.W. and Holland, N.D. (2007). "Endogenous green fluorescent protein (GFP) in amphioxus." Biol Bull 213(2): 95-100.

Delagrave, S., Hawtin, R.E., Silva, C.M., Yang, M.M. and Youvan, D.C. (1995). "Red-shifted excitation mutants of the green fluorescent protein." Biotechnology (N Y) 13(2): 151-154.

DiCiommo, D.P. and Bremner, R. (1998). "Rapid, high level protein production using DNA-based Semliki Forest virus vectors." J Biol Chem 273(29): 18060-18066.

Dittrich, P.S. and Schwille, P. (2001). "Photobleaching and stabilization of fluorophores used for single-molecule analysis with one- and two-photon excitation." Appl Phys B 73: 829-837.

Donnert, G., Eggeling, C. and Hell, S.W. (2007). "Major signal increase in fluorescence microscopy through dark-state relaxation." Nat Methods 4(1): 81-86.

Eggeling, C., Hilbert, M., Bock, H., Ringemann, C., Hofmann, M., Stiel, A.C., Andresen, M., Jakobs, S., Egner, A., Schonle, A. and Hell, S.W. (2007). "Reversible photoswitching enables single-molecule fluorescence fluctuation spectroscopy at high molecular concentration." Microsc Res Tech 70(12): 1003-1009.

Eggeling, C., Widengren, J., Rigler, R. and Seidel, C.A.M. (1998). "Photobleaching of fluorescent dyes under conditions used for singlemolecule detection: Evidence of two-step photolysis." Anal Chem 70: 2651-2659.

Eggeling, C., Widengren, J., Rigler, R. and Seidel, C.A.M. (1999). Photostabilities of fluorescent dyes for single-molecule spectroscopy: Mechanisms and experimental methods for estimating photobleaching in aqueous solution. Applied fluorescence in chemistry, biology and medicine. W. Rettig, B. Strehmel, M. Schrader and H. Seifert. Berlin, Springer: 193-240

Egner, A., Geisler, C., von Middendorff, C., Bock, H., Wenzel, D., Medda, R., Andresen, M., Stiel, A.-C., Jakobs, S., Eggeling, C., Schoenle, A. and Hell, S.W. (2007). "Fluorescence nanoscopy in whole cells by asnychronous localization of photoswitching emitters." Biophys J 93: 3285-3290.

Egner, A., Geisler, C., von Middendorff, C., Bock, H., Wenzel, D., Medda, R., Andresen, M., Stiel, A.C., Jakobs, S., Eggeling, C., Schonle, A. and Hell, S.W. (2007). "Fluorescence nanoscopy in whole cells by asnychronous localization of photoswitching emitters." Biophys J 93(9): 3285-3290.

Ehrenberg, M. and Rigler, R. (1974). "Rotational Brownian Motion and Fluorescence Intensity Fluctuations." Chem Phys 4(3): 390-401.

Elowitz, M.B., Surette, M.G., Wolf, P.E., Stock, J. and Leibler, S. (1997). "Photoactivation turns green fluorescent protein red." Curr Biol 7(10): 809812.

Erez, Y., Gepshtein, R., Presiado, I., Trujillo, K., Kallio, K., Remington, S.J. and Huppert, D. (2011). "Structure and excited-state proton transfer in the GFP S205A mutant." J Phys Chem B 115(41): 11776-11785. 
Evdokimov, A.G., Pokross, M.E., Egorov, N.S., Zaraisky, A.G., Yampolsky, I.V., Merzlyak, E.M., Shkoporov, A.N., Sander, I., Lukyanov, K.A. and Chudakov, D.M. (2006). "Structural basis for the fast maturation of Arthropoda green fluorescent protein." EMBO Rep 7(10): 1006-1012.

Flors, C., Hotta, J., Uji-i, H., Dedecker, P., Ando, R., Mizuno, H., Miyawaki, A. and Hofkens, J. (2007). "A stroboscopic approach for fast photoactivationlocalization microscopy with Dronpa mutants." J Am Chem Soc 129(45): $13970-13977$.

Fölling, J., Bossi, M., Bock, H., Medda, R., Wurm, C.A., Hein, B., Jakobs, S., Eggeling, C. and Hell, S.W. (2008). "Fluorescence nanoscopy by groundstate depletion and single-molecule return." Nat Methods 5: 943-945.

Fourkas, J.T. (2010). "Nanoscale Photolithography with Visible Light." J Phys Chem Lett 1: 1221-1227.

Fradkov, A.F., Chen, Y., Ding, L., Barsova, E.V., Matz, M.V. and Lukyanov, S.A. (2000). "Novel fluorescent protein from Discosoma coral and its mutants possesses a unique far-red fluorescence." FEBS Lett 479(3): 127-130.

Gahwiler, B.H., Capogna, M., Debanne, D., McKinney, R.A. and Thompson, S.M. (1997). "Organotypic slice cultures: a technique has come of age." Trends Neurosci 20(10): 471-477.

Godley, B.F., Shamsi, F.A., Liang, F.Q., Jarrett, S.G., Davies, S. and Boulton, M. (2005). "Blue light induces mitochondrial DNA damage and free radical production in epithelial cells." J Biol Chem 280(22): 21061-21066.

Griesbeck, O., Baird, G.S., Campbell, R.E., Zacharias, D.A. and Tsien, R.Y. (2001). "Reducing the environmental sensitivity of yellow fluorescent protein - Mechanism and applications." J Biol Chem 276(31): 2918829194.

Gross, L.A., Baird, G.S., Hoffman, R.C., Baldridge, K.K. and Tsien, R.Y. (2000). "The structure of the chromophore within DsRed, a red fluorescent protein from coral." Proc Natl Acad Sci USA 97(22): 11990-11995.

Grotjohann, T., Testa, I., Leutenegger, M., Bock, H., Urban, N.T., LavoieCardinal, F., Willig, K.I., Eggeling, C., Jakobs, S. and Hell, S.W. (2011). "Diffraction-unlimited all-optical imaging and writing with a photochromic GFP." Nature 478(7368): 204-208.

Gurskaya, N.G., Verkhusha, V.V., Shcheglov, A.S., Staroverov, D.B., Chepurnykh, T.V., Fradkov, A.F., Lukyanov, S. and Lukyanov, K.A. (2006). "Engineering of a monomeric green-to-red photoactivatable fluorescent protein induced by blue light." Nat Biotechnol 24(4): 461-465.

Gustafsson, M.G. (2005). "Nonlinear structured-illumination microscopy: widefield fluorescence imaging with theoretically unlimited resolution." Proc Natl Acad Sci USA 102(37): 13081-13086.

Haas, J., Park, E.C. and Seed, B. (1996). "Codon usage limitation in the expression of HIV-1 envelope glycoprotein." Curr Biol 6(3): 315-324.

Hanahan, D. (1983). "Studies on transformation of Escherichia coli with plasmids." J Mol Biol 166(4): 557-580.

Harke, B., Ullal, C.K., Keller, J. and Hell, S.W. (2008). "Three-Dimensional Nanoscopy of Colloidal Crystals." Nano Lett 8(5): 1309-1313.

Haustein, E. and Schwille, P. (2003). "Ultrasensitive investigations of biological systems by fluorescence correlation spectroscopy." Methods 29(2): 153166. 
Heikal, A.A., Hess, S.T., Baird, G.S., Tsien, R.Y. and Webb, W.W. (2000). "Molecular spectroscopy and dynamics of intrinsically fluorescent proteins: Coral red (dsRed) and yellow (Citrine)." Proc Natl Acad Sci USA 97(22): 11996-12001.

Heim, R., Cubitt, A.B. and Tsien, R.Y. (1995). "Improved green fluorescence." Nature 373(6516): 663-664.

Heim, R., Prasher, D.C. and Tsien, R.Y. (1994). "Wavelength mutations and posttranslational autoxidation of green fluorescent protein." Proc Natl Acad Sci USA 91(26): 12501-12504.

Hein, B., Willig, K.I. and Hell, S.W. (2008). "Stimulated emission depletion (sted) nanoscopy of a fluorescent protein-labeled organelle inside a living cell." Proc Natl Acad Sci USA 105(38): 14271-14276.

Hein, B., Willig, K.I., Wurm, C.A., Westphal, V., Jakobs, S. and Hell, S.W. (2010). "Stimulated Emission Depletion Nanoscopy of Living Cells Using SNAPTag Fusion Proteins." Biophys J 98: 158 - 163.

Heintzmann, R., Jovin, T.M. and Cremer, C. (2002). "Saturated patterned excitation microscopy - A concept for optical resolution improvement." $\underline{J}$ Opt Soc Am A Opt Image Sci Vis 19(8): 1599-1609.

Heisenberg, W. (1930). The physical principles of the quantum theory. Chicago, Chicago Univ Press.

Hell, S.W. (2003). "Toward fluorescence nanoscopy." Nat Biotechnol 21(11): 1347-1355.

Hell, S.W. (2004). "Strategy for far-field optical imaging and writing without diffraction limit." Phys Lett A 326(1-2): 140-145.

Hell, S.W. (2007). "Far-Field Optical Nanoscopy." Science 316(5828): 11531158.

Hell, S.W. (2009). "Microscopy and its focal switch." Nat Methods 6(1): 24-32.

Hell, S.W. and Andresen, V. (2001). "Space-multiplexed multifocal nonlinear microscopy." J Microsc 202: 457-463.

Hell, S.W., Jakobs, S. and Kastrup, L. (2003). "Imaging and writing at the nanoscale with focused visible light through saturable optical transitions." Appl Phys A 77: 859-860.

Hell, S.W. and Kroug, M. (1995). "Ground-state depletion fluorescence microscopy, a concept for breaking the diffraction resolution limit." Appl Phys B 60: 495-497.

Hell, S.W., Schmidt, R. and Egner, A. (2009). "Diffraction-unlimited threedimensional optical nanoscopy with opposing lenses." Nat Photonics 3: 381-387.

Hell, S.W. and Wichmann, J. (1994). "Breaking the diffraction resolution limit by stimulated-emission - stimulated-emission-depletion fluorescence microscopy." Opt Lett 19(11): 780-782.

Henderson, J.N., Ai, H.W., Campbell, R.E. and Remington, S.J. (2007). "Structural basis for reversible photobleaching of a green fluorescent protein homologue." Proc Natl Acad Sci USA 104(16): 6672-6677.

Henderson, J.N. and Remington, S.J. (2005). "Crystal structures and mutational analysis of amFP486, a cyan fluorescent protein from Anemonia majano." Proc Natl Acad Sci USA 102(36): 12712-12717.

Hess, S.T., Girirajan, T.P. and Mason, M.D. (2006). "Ultra-high resolution imaging by fluorescence photoactivation localization microscopy." Biophys J 91(11): 4258-4272. 
Hirschberg, Y. (1956). "Reversible formation and eradication of colors by irradiation at low temperatures a photochemical memory model." $\underline{\mathrm{J} \mathrm{Am}}$ Chem Soc 78: 2304-2312.

Hofmann, M., Eggeling, C., Jakobs, S. and Hell, S.W. (2005). "Breaking the diffraction barrier in fluorescence microscopy at low light intensities by using reversibly photoswitchable proteins." Proc Natl Acad Sci USA 102(49): 17565-17569.

Hooke, R. (1665). Micrographia.

Hopt, A. and Neher, E. (2001). "Highly nonlinear photodamage in two-photon fluorescence microscopy." Biophys J 80: 2029-2036.

Huang, B., Babcock, H. and Zhuang, X. (2010). "Breaking the diffraction barrier: super-resolution imaging of cells." Cell 143: 1047-1058.

Hunt, M.E., Scherrer, M.P., Ferrari, F.D. and Matz, M.V. (2010). "Very bright green fluorescent proteins from the Pontellid copepod Pontella mimocerami." PLoS One 5(7): e11517.

Immink, K.A.S. (1995). "EFMPlus: The coding format of the high-density compact disc." leee 1995 International Conference on Consumer Electronics Digest of Technical Papers: 80-81.

Inouye, S. and Tsuji, F.I. (1994). "Aequorea green fluorescent protein. Expression of the gene and fluorescence characteristics of the recombinant protein." FEBS Lett 341(2-3): 277-280.

Irie, M. (2000). "Diarylethenes for memories and switches." Chemistry Reviews 100(5): 1685-1716.

Irie, M., Fukaminato, T., Sasaki, T., Tamai, N. and Kawai, T. (2002). "Organic chemistry: a digital fluorescent molecular photoswitch." Nature 420(6917): 759-760.

Jablonski, A. (1935). "Ueber den Mechanismus der Photolumineszenz von Farbstoffphosphoren." Z Phys 94: 38-46.

Jablonski, A. and Szymanowski, W. (1935). "Thermal Rotations of Fluorescence Molecules and Duration of Luminiscence." Nature 135: 582.

Jakobs, S., Schauss, A.C. and Hell, S.W. (2003). "Photoconversion of matrix targeted GFP enables analysis of continuity and intermixing of the mitochondrial lumen." FEBS Lett 554: 194-200.

Jakobs, S., Subramaniam, V., Schonle, A., Jovin, T.M. and Hell, S.W. (2000). "EFGP and DsRed expressing cultures of Escherichia coli imaged by confocal, two-photon and fluorescence lifetime microscopy." FEBS Lett 479(3): 131-135.

Kask, P., Palo, K., Ullmann, D. and Gall, K. (1999). "Fluorescence-intensity distribution analysis and its application in biomolecular detection technology." Proc Natl Acad Sci USA 96: 13756-13761.

Kawata, S. and Kawata, Y. (2000). "Three-Dimensional Optical Data Storage Using Photochromic Materials." Chem Rev 100(5): 1777-1788.

Kirkland, A.I. and Hutchinsons, L. (2007). Atomic resolution transmission electron microscopy. Science of Microscopy. P. W. Hawkes and J. C. Spence, Springer. 1: 3-64.

Labas, Y.A., Gurskaya, N.G., Yanushevich, Y.G., Fradkov, A.F., Lukyanov, K.A., Lukyanov, S.A. and Matz, M.V. (2002). "Diversity and evolution of the green fluorescent protein family." Proc Natl Acad Sci USA 99(7): 42564261. 
Lamesch, P., Li, N., Milstein, S., Fan, C.Y., Hao, T., Szabo, G., Hu, Z.J., Venkatesan, K., Bethel, G., Martin, P., Rogers, J., Lawlor, S., McLaren, S., Dricot, A., Borick, H., Cusick, M.E., Vandenhaute, J., Dunham, I., Hill, D.E. and Vidal, M. (2007). "h0rfeome v3.1: a resource of human open reading frames representing over 10,000 human genes." Genomics 89(3): 307315.

Leung, D.W., Chen, E. and Goeddel, D.V. (1989). "A method for random mutagenesis of a defined DNA segment using a modified polymerase chain reaction." Technique 1: 11-15.

Lill, M.A. and Helms, V. (2002). "Proton shuttle in green fluorescent protein studied by dynamic simulations." Proc Natl Acad Sci USA 99(5): 27782781.

Lin, M.Z., McKeown, M.R., Ng, H.L., Aguilera, T.A., Shaner, N.C., Campbell, R.E., Adams, S.R., Gross, L.A., Ma, W., Alber, T. and Tsien, R.Y. (2009). "Autofluorescent proteins with excitation in the optical window for intravital imaging in mammals." Chem Biol 16(11): 1169-1179.

Lucy, L.B. (1974). "An iterative technique for rectification of observed distributions." Astron J 79: 745-754.

Lukyanov, K.A., Fradkov, A.F., Gurskaya, N.G., Matz, M.V., Labas, Y.A., Savitsky, A.P., Markelov, M.L., Zaraisky, A.G., Zhao, X., Fang, Y., Tan, W. and Lukyanov, S.A. (2000). "Natural animal coloration can Be determined by a nonfluorescent green fluorescent protein homolog." $\mathrm{J}$ Biol Chem 275(34): 25879-25882.

Magde, D., Webb, W.W. and Elson, E. (1972). "Thermodynamic fluctuations in a reacting system - measurement by fluorescence correlation spectroscopy." Phys Rev Lett 29(11): 705-708.

Malashkevich, V.N., Subach, O.M., Almo, S.C. and Verkhusha, V.V. (2010). "Crystal structure of TagRFP fluorescent protein." To be published PDB-ID $3 \mathrm{M} 22$

Matz, M.V., Fradkov, A.F., Labas, Y.A., Savitsky, A.P., Zaraisky, A.G., Markelov, M.L. and Lukyanov, S.A. (1999). "Fluorescent proteins from nonbioluminescent Anthozoa species." Nat Biotechnol 17(10): 969-973.

Matz, M.V., Labas, Y.A. and Ugalde, J. (2006). "Evolution of function and color in GFP-like proteins." Methods Biochem Anal 47: 139-161.

Menon, R. and Smith, H.I. (2006). "Absorbance-modulation optical lithography." $\underline{J}$ Opt Soc Am A Opt Image Sci Vis 23(9): 2290-2294.

Merzlyak, E.M., Goedhart, J., Shcherbo, D., Bulina, M.E., Shcheglov, A.S., Fradkov, A.F., Gaintzeva, A., Lukyanov, K.A., Lukyanov, S., Gadella, T.W. and Chudakov, D.M. (2007). "Bright monomeric red fluorescent protein with an extended fluorescence lifetime." Nat Methods 4(7): 555-557.

Mizuno, H., Mal, T.K., Tong, K.I., Ando, R., Furuta, T., Ikura, M. and Miyawaki, A. (2003). "Photo-induced peptide cleavage in the green-to-red conversion of a fluorescent protein." Mol Cell 12(4): 1051-1058.

Mizuno, H., Mal, T.K., Walchli, M., Kikuchi, A., Fukano, T., Ando, R., Jeyakanthan, J., Taka, J., Shiro, Y., Ikura, M. and Miyawaki, A. (2008). "Light-dependent regulation of structural flexibility in a photochromic fluorescent protein." Proc Natl Acad Sci USA 105(27): 9227-9232.

Moerner, W.E. (2002). "A dozen years of single-molecule spectroscopy in physics, chemistry, and biophysics." Journal of Physical Chemistry B 106(5): 910-927. 
Morise, H., Shimomura, O., Johnson, F.H. and Winant, J. (1974). "Intermolecular energy transfer in the bioluminescent system of Aequorea." Biochemistry 13(12): 2656-2662.

Morozova, K.S., Piatkevich, K.D., Gould, T.J., Zhang, J., Bewersdorf, J. and Verkhusha, V.V. (2010). "Far-red fluorescent protein excitable with red lasers for flow cytometry and superresolution STED nanoscopy." Biophys J 99(2): L13-15.

Nam, K.H., Kwon, O.Y., Sugiyama, K., Lee, W.H., Kim, Y.K., Song, H.K., Kim, E.E., Park, S.Y., Jeon, H. and Hwang, K.Y. (2007). "Structural characterization of the photoswitchable fluorescent protein Dronpa-C62S." Biochem Biophys Res Commun 354(4): 962-967.

Nienhaus, G.U., Nienhaus, K., Holzle, A., Ivanchenko, S., Renzi, F., Oswald, F., Wolff, M., Schmitt, F., Rocker, C., Vallone, B., Weidemann, W., Heilker, R., Nar, H. and Wiedenmann, J. (2006). "Photoconvertible fluorescent protein EosFP: biophysical properties and cell biology applications." Photochem Photobiol 82(2): 351-358.

Nienhaus, K., Nienhaus, G.U., Wiedenmann, J. and Nar, H. (2005). "Structural basis for photo-induced protein cleavage and green-to-red conversion of fluorescent protein EosFP." Proc Natl Acad Sci USA 102(26): 9156-9159.

Niwa, H., Inouye, S., Hirano, T., Matsuno, T., Kojima, S., Kubota, M., Ohashi, M. and Tsuji, F.I. (1996). "Chemical nature of the light emitter of the Aequorea green fluorescent protein." Proc Natl Acad Sci USA 93(24): 13617-13622.

Ormo, M., Cubitt, A.B., Kallio, K., Gross, L.A., Tsien, R.Y. and Remington, S.J. (1996). "Crystal structure of the Aequorea victoria green fluorescent protein." Science 273(5280): 1392-1395.

Pakhomov, A.A. and Martynov, V.I. (2009). "Posttranslational chemistry of proteins of the GFP family." Biochemistry (Mosc) 74(3): 250-259.

Palm, G.J., Zdanov, A., Gaitanaris, G.A., Stauber, R., Pavlakis, G.N. and Wlodawer, A. (1997). "The structural basis for spectral variations in green fluorescent protein." Nat Struct Biol 4(5): 361-365.

Patterson, G., Davidson, M., Manley, S. and Lippincott-Schwartz, J. (2010). "Superresolution imaging using single-molecule localization." Annu Rev Phys Chem 61: 345-367.

Patterson, G.H., Knobel, S.M., Sharif, W.D., Kain, S.R. and Piston, D.W. (1997). "Use of the green fluorescent protein and its mutants in quantitative fluorescence microscopy." Biophys J 73: 2782-2790.

Patterson, G.H. and Lippincott-Schwartz, J. (2002). "A Photoactivatable GFP for Selective Photolabeling of Proteins and Cells." Science 297(5588): 18731877.

Pawley, J.B. (2006). Handbook of biological confocal microscopy. New York, Springer.

Peak, J.G. and Peak, M.J. (1990). "Ultraviolet light induces double-strand breaks in DNA of cultured human P3 cells as measured by neutral filter elution." Photochem Photobiol 52(2): 387-393.

Petersen, J., Wilmann, P.G., Beddoe, T., Oakley, A.J., Devenish, R.J., Prescott, M. and Rossjohn, J. (2003). "The 2.0-angstrom crystal structure of eqfp611, a far red fluorescent protein from the sea anemone entacmaea quadricolor." J Biol Chem 278(45): 44626-44631. 
Pfennig, N. and Wagener, S. (1986). "An improved method of preparing wet mounts for photomicrographs of microorganisms." J. Microbiol. Meth. 4: 303-306.

Pouwels, L.J., Zhang, L., Chan, N.H., Dorrestein, P.C. and Wachter, R.M. (2008). "Kinetic isotope effect studies on the de novo rate of chromophore formation in fast- and slow-maturing GFP variants." Biochemistry 47(38): 10111-10122.

Prasher, D.C., Eckenrode, V.K., Ward, W.W., Prendergast, F.G. and Cormier, M.J. (1992). "Primary structure of the Aequorea victoria green-fluorescent protein." Gene 111(2): 229-233.

Prescott, M., Ling, M., Beddoe, T., Oakley, A.J., Dove, S., Hoegh-Guldberg, O., Devenish, R.J. and Rossjohn, J. (2003). "The 2.2 A crystal structure of a pocilloporin pigment reveals a nonplanar chromophore conformation." Structure 11(3): 275-284.

Rego, E.H., Shao, L., Macklin, J.J., Winoto, L., Johansson, G.A., Kamps-Hughes, N., Davidson, M.W. and Gustafsson, M.G. (2011). "Nonlinear structuredillumination microscopy with a photoswitchable protein reveals cellular structures at 50-nm resolution." Proc Natl Acad Sci USA. 109(3): E135143

Reid, B.G. and Flynn, G.C. (1997). "Chromophore formation in green fluorescent protein." Biochemistry 36(22): 6786-6791.

Reynolds, F.W. and Hesbacher, E.N. (1950). "Darkfield microscopy; some principles and applications." J Vener Dis Inf 31(1): 17-23.

Richardson, W.H. (1972). "Bayesian-based iterative method of image restoration." J Opt Soc Am 62: 55-59.

Riedl, J., Crevenna, A.H., Kessenbrock, K., Yu, J.H., Neukirchen, D., Bista, N., Bradke, F., Jenne, D., Holak, T.A., Werb, Z., Sixt, M. and WedlichSoldner, R. (2008). "Lifeact: a versatile marker to visualize F-actin." Nat Methods 5(7): 605-607.

Rosenow, M.A., Patel, H.N. and Wachter, R.M. (2005). "Oxidative chemistry in the GFP active site leads to covalent cross-linking of a modified leucine side chain with a histidine imidazole: implications for the mechanism of chromophore formation." Biochemistry 44(23): 8303-8311.

Royant, A. and Noirclerc-Savoye, M. (2011). "Stabilizing role of glutamic acid 222 in the structure of Enhanced Green Fluorescent Protein." J Struct Biol 174(2): 385-390.

Rust, M.J., Bates, M. and Zhuang, X.W. (2006). "Sub-diffraction-limit imaging by stochastic optical reconstruction microscopy (STORM)." Nat Methods 3: 793-795.

Sawano, A. and Miyawaki, A. (2000). "Directed evolution of green fluorescent protein by a new versatile PCR strategy for site-directed and semi-random mutagenesis." Nucleic Acids Res 28(16): E78.

Sawin, K.E. and Nurse, P. (1997). "Photoactivation of green fluorescent protein." Curr Biol 7(10): R606-607.

Schäfer, L.V., Groenhof, G., Klingen, A.R., Ullmann, G.M., Boggio-Pasqua, M., Robb, M.A. and Grubmüller, H. (2007). "Photoswitching of the fluorescent protein asFP595: mechanism, proton pathways, and absorption spectra." Angew Chem Int Ed Engl 46(4): 530-536. 
Schenk, A., Ivanchenko, S., Rocker, C., Wiedenmann, J. and Nienhaus, G.U. (2004). "Photodynamics of red fluorescent proteins studied by fluorescence correlation spectroscopy." Biophys J 86(1 Pt 1): 384-394.

Schmidt, R., Wurm, C.A., Jakobs, S., Engelhardt, J., Egner, A. and Hell, S.W. (2008). "Spherical nanosized focal spot unravels the interior of cells." Nat Methods 5(6): 539-544.

Schmidt, R., Wurm, C.A., Punge, A., Egner, A., Jakobs, S. and Hell, S.W. (2009). "Mitochondrial Cristae Revealed with Focused Light." Nano Lett 9(6): 2508-2510.

Schuettrigkeit, T., Till von Feilitzsch, Christian K. Kompa, Konstantin A. Lukyanov, Alexander P. Savitsky, Alexander A. Voityuk, Maria E. MichelBeyerle (2006). "Femtosecond study of light-induced fluorescence increase of the dark chromoprotein asFP595." Chem Phys 323(2-3): 149160.

Schwentker, M.A., Bock, H., Hofmann, M., Jakobs, S., Bewersdorf, J., Eggeling, C. and Hell, S.W. (2007). "Wide-field subdiffraction RESOLFT microscopy using fluorescent protein photoswitching." Microsc Res Tech 70(3): 269280.

Shagin, D.A., Barsova, E.V., Yanushevich, Y.G., Fradkov, A.F., Lukyanov, K.A., Labas, Y.A., Semenova, T.N., Ugalde, J.A., Meyers, A., Nunez, J.M., Widder, E.A., Lukyanov, S.A. and Matz, M.V. (2004). "GFP-like proteins as ubiquitous metazoan superfamily: evolution of functional features and structural complexity." Mol Biol Evol 21(5): 841-850.

Shaner, N.C., Campbell, R.E., Steinbach, P.A., Giepmans, B.N., Palmer, A.E. and Tsien, R.Y. (2004). "Improved monomeric red, orange and yellow fluorescent proteins derived from Discosoma $\mathrm{sp}$. red fluorescent protein." Nat Biotechnol 22(12): 1567-1572.

Shaner, N.C., Lin, M.Z., McKeown, M.R., Steinbach, P.A., Hazelwood, K.L., Davidson, M.W. and Tsien, R.Y. (2008). "Improving the photostability of bright monomeric orange and red fluorescent proteins." Nat Methods 5(6): 545-551.

Shaner, N.C., Patterson, G.H. and Davidson, M.W. (2007). "Advances in fluorescent protein technology." J Cell Sci 120(Pt 24): 4247-4260.

Shaner, N.C., Steinbach, P.A. and Tsien, R.Y. (2005). "A guide to choosing fluorescent proteins." Nat Methods 2(12): 905-909.

Shimomura, O., Johnson, F.H. and Saiga, Y. (1961). "Purification and properties of Cypridina luciferase." J Cell Comp Physiol 58: 113-123.

Shu, X., Shaner, N.C., Yarbrough, C.A., Tsien, R.Y. and Remington, S.J. (2006). "Novel chromophores and buried charges control color in mFruits." Biochemistry 45(32): 9639-9647.

Sniegowski, J.A., Lappe, J.W., Patel, H.N., Huffman, H.A. and Wachter, R.M. (2005). "Base catalysis of chromophore formation in Arg96 and Glu222 variants of green fluorescent protein." J Biol Chem 280(28): 26248-26255.

Song, L., Hennink, E.J., Young, I.T. and Tanke, H.J. (1995). "Photobleaching kinetics of fluorescein in quantitative fluorescence microscopy." Biophys J 68(6): 2588-2600.

Stiel, A.C., Andresen, M., Bock, H., Hilbert, M., Schilde, J., Schonle, A., Eggeling, C., Egner, A., Hell, S.W. and Jakobs, S. (2008). "Generation of Monomeric Reversibly Switchable Red Fluorescent Proteins for Far-Field Fluorescence Nanoscopy." Biophys J 95: 2989-2997. 
Stiel, A.C., Trowitzsch, S., Weber, G., Andresen, M., Eggeling, C., Hell, S.W., Jakobs, S. and Wahl, M.C. (2007). "1.8 angstrom bright-state structure of the reversibly switchable fluorescent protein dronpa guides the generation of fast switching variants." Biochem J 402(1): 35-42.

Strack, R.L., Strongin, D.E., Mets, L., Glick, B.S. and Keenan, R.J. (2010). "Chromophore formation in DsRed occurs by a branched pathway." J Am Chem Soc 132(24): 8496-8505.

Strongin, D.E., Bevis, B., Khuong, N., Downing, M.E., Strack, R.L., Sundaram, K., Glick, B.S. and Keenan, R.J. (2007). "Structural rearrangements near the chromophore influence the maturation speed and brightness of DsRed variants." Protein Eng Des Sel 20(11): 525-534.

Subach, F.V., Patterson, G.H., Manley, S., Gillette, J.M., Lippincott-Schwartz, J. and Verkhusha, V.V. (2009). "Photoactivatable mcherry for high-resolution two-color fluorescence microscopy." Nat Methods 6(2): 311-311.

Subach, F.V., Patterson, G.H., Renz, M., Lippincott-Schwartz, J. and Verkhusha, V.V. (2010). "Bright Monomeric Photoactivatable Red Fluorescent Protein for Two-Color Super-Resolution sptPALM of Live Cells." J Am Chem Soc 132(18): 6481-6491.

Subach, O.M., Gundorov, I.S., Yoshimura, M., Subach, F.V., Zhang, J., Gruenwald, D., Souslova, E.A., Chudakov, D.M. and Verkhusha, V.V. (2008). "Conversion of red fluorescent protein into a bright blue probe." Chem Biol 15(10): 1116-1124.

Testa, I., Schonle, A., Middendorff, C.V., Geisler, C., Medda, R., Wurm, C.A., Stiel, A.C., Jakobs, S., Bossi, M., Eggeling, C., Hell, S.W. and Egner, A. (2008). "Nanoscale separation of molecular species based on their rotational mobility." Opt Express 16(25): 21093-21104.

Thastrup, O., Tullin, S., Kongsbak Poulsen, L. and Bjørn, S. (1995). Fluorescent proteins. US Patent.

Tomosugi, W., Matsuda, T., Tani, T., Nemoto, T., Kotera, I., Saito, K., Horikawa, K. and Nagai, T. (2009). "An ultramarine fluorescent protein with increased photostability and pH insensitivity." Nat Methods 6(5): 351-353.

Tsien, R.Y. (1998). "The green fluorescent protein." Annu Rev Biochem 67(1): 509-544.

Tubbs, J.L., Tainer, J.A. and Getzoff, E.D. (2005). "Crystallographic structures of Discosoma red fluorescent protein with immature and mature chromophores: linking peptide bond trans-cis isomerization and acylimine formation in chromophore maturation." Biochemistry 44(29): 9833-9840.

van Thor, J.J., Georgiev, G.Y., Towrie, M. and Sage, J.T. (2005). "Ultrafast and low barrier motions in the photoreactions of the green fluorescent protein." J Biol Chem 280(39): 33652-33659.

van Thor, J.J. and Sage, J.T. (2006). "Charge transfer in green fluorescent protein." Photochem Photobiol Sci 5(6): 597-602.

van Thor, J.J., Zanetti, G., Ronayne, K.L. and Towrie, M. (2005). "Structural events in the photocycle of green fluorescent protein." $\underline{J}$ Phys Chem B 109(33): 16099-16108.

Vats, P. and Rothfield, L. (2007). "Duplication and segregation of the actin (MreB) cytoskeleton during the prokaryotic cell cycle." Proc Natl Acad Sci USA 104(45): 17795-17800. 
Verkhusha, V.V., Chudakov, D.M., Gurskaya, N.G., Lukyanov, S. and Lukyanov, K.A. (2004). "Common pathway for the red chromophore formation in fluorescent proteins and chromoproteins." Chem Biol 11(6): 845-854.

Verkhusha, V.V. and Sorkin, A. (2005). "Conversion of the monomeric red fluorescent protein into a photoactivatable probe." Chem Biol 12(3): 279285.

Vogelsang, J., Kasper, R., Steinhauer, C., Person, B., Heilemann, M., Sauer, M. and Tinnefeld, P. (2008). "A Reducing and Oxidizing System Minimizes Photobleaching and Blinking of Fluorescent Dyes." Angew Chem Int Ed 47(29): 5465-5469.

Wachter, R.M., King, B.A., Heim, R., Kallio, K., Tsien, R.Y., Boxer, S.G. and Remington, S.J. (1997). "Crystal structure and photodynamic behavior of the blue emission variant $\mathrm{Y} 66 \mathrm{H} / \mathrm{Y} 145 \mathrm{~F}$ of green fluorescent protein." Biochemistry 36(32): 9759-9765.

Wachter, R.M., Watkins, J.L. and Kim, H. (2010). "Mechanistic diversity of red fluorescence acquisition by GFP-like proteins." Biochemistry 49(35): 74177427.

Wang, L., Jackson, W.C., Steinbach, P.A. and Tsien, R.Y. (2004). "Evolution of new nonantibody proteins via iterative somatic hypermutation." Proc Natl Acad Sci USA 101(48): 16745-16749.

Wang, S. and Hazelrigg, T. (1994). "Implications for bcd mRNA localization from spatial distribution of exu protein in Drosophila oogenesis." Nature 369(6479): 400-403.

Westphal, V., Rizzoli, S.O., Lauterbach, M.A., Kamin, D., Jahn, R. and Hell, S.W. (2008). "Video-Rate Far-Field Optical Nanoscopy Dissects Synaptic Vesicle Movement." Science 320(5873): 246-249.

Widengren, J., Mets, U. and Rigler, R. (1995). "Fluorescence correlation spectroscopy of triplet-states in solution - a theoretical and experimentalstudy." J Phys Chem 99: 13368-13379.

Wiedenmann, J., Ivanchenko, S., Oswald, F., Schmitt, F., Rocker, C., Salih, A., Spindler, K.D. and Nienhaus, G.U. (2004). "EosFP, a fluorescent marker protein with UV-inducible green-to-red fluorescence conversion." Proc Natl Acad Sci USA 101(45): 15905-15910.

Wiehler, J., Jung, G., Seebacher, C., Zumbusch, A. and Steipe, B. (2003). "Mutagenic stabilization of the photocycle intermediate of green fluorescent protein (GFP)." Chembiochem 4(11): 1164-1171.

Yan, W., Xie, D. and Zeng, J. (2009). "The 559-to-600 nm shift observed in red fluorescent protein eqFP611 is attributed to cis-trans isomerization of the chromophore in an anionic protein pocket." Phys Chem Chem Phys 11(29): 6042-6050.

Yarbrough, D., Wachter, R.M., Kallio, K., Matz, M.V. and Remington, S.J. (2001). "Refined crystal structure of dsred, a red fluorescent protein from coral, at 2.0-angstrom resolution." Proc Natl Acad Sci USA 98(2): 462-467.

Yokoyama, Y. (2000). "Fulgides for Memories and Switches." Chemistry Reviews 100(5): 1717-1740.

Zacharias, D.A., Violin, J.D., Newton, A.C. and Tsien, R.Y. (2002). "Partitioning of Lipid-Modified Monomeric GFPs into Membrane Microdomains of Live Cells." Science 296: 913-916.

Zernike, F. (1935). "The phase contrast process in microscopic examinations." Physikalische Zeitschrift 36: 848-851. 
Zhang, L., Patel, H.N., Lappe, J.W. and Wachter, R.M. (2006). "Reaction progress of chromophore biogenesis in green fluorescent protein." $\underline{\mathrm{J} \mathrm{Am}}$ Chem Soc 128(14): 4766-4772. 


\section{List of Figures}

\begin{tabular}{llr}
\hline Figure II.1 & Excitation and emission spectrum of AlexaFluor 488 & 7 \\
\hline Figure II.2 & The Jablonski diagram & 7 \\
\hline Figure II.3 & $\begin{array}{l}\text { Scheme of a classical epifluorescence microscope } \\
\text { setup and a CLSM setup }\end{array}$ & 10 \\
\hline Figure II.4 & The STED microscope & 12 \\
\hline Figure II.5 & The principle of SMS microscopy & 14 \\
\hline Figure II.6 & The jellyfish Aequoria victoria & 15 \\
\hline Figure II.7 & The protein structure of fluorescent proteins & 17 \\
\hline Figure II.8 & $\begin{array}{l}\text { Maturation model of GFP and DsRed type } \\
\text { chromophores }\end{array}$ & 19 \\
\hline Figure II.9 & Protonation states and ESPT in avGFP & 21 \\
\hline Figure II.10 & $\begin{array}{l}\text { The emission wavelengths of different fluorescent } \\
\text { proteins }\end{array}$ & 23 \\
\hline Figure II.11 & The chromophore structure of BFP, CFP and YFP & 23 \\
\hline Figure II.12 & Structural basis of photoactivation & 27 \\
\hline Figure II.13 & Structural basis of green to red photoconversion & 28 \\
\hline Figure II.14 & Switching modes in RSFPs & 30 \\
\hline Figure II.15 & Chromophore structures of Dronpa and asFP595 & 30 \\
\hline
\end{tabular}

\begin{tabular}{llr}
\hline Figure IV.1 & A laser-based screening setup & 36 \\
\hline Figure IV.2 & Switching characteristics of RSFPs & 39 \\
\hline Figure IV.3 & Amino acid sequence of EGFP and rsEGFP & 41 \\
\hline Figure IV.4 & Spectral properties of rsEGFP & 42 \\
\hline Figure IV.5 & Relaxation kinetic of rs EGFP & 42 \\
\hline Figure IV.6 & $\begin{array}{l}\text { Absorption spectra of equilibrium-state rsEGFP at } \\
\text { different pH values }\end{array}$ & 42 \\
\hline Figure IV.7 & rsEGFP is a monomer & 43 \\
\hline Figure IV.8 & Maturation kinetic of rsEGFP at 37 ${ }^{\circ}$ C & 43 \\
\hline Figure IV.9 & $\begin{array}{l}\text { Expression of various functional rsEGFP fusion } \\
\text { proteins in mammalian PtK2 Cells }\end{array}$ & 44 \\
\hline Figure IV.10 & $\begin{array}{l}\text { Switching of Keartin19-rsEGFP fusion proteins } \\
\text { expressed in living PtK2 cells }\end{array}$ & 45 \\
\hline Figure IV.11 & Switching curves of Dronpa and rsEGFP & 46 \\
\hline Figure IV.12 & $\begin{array}{l}\text { Switching fatigue and bleaching characteristics of } \\
\text { Dronpa and rsEGFP }\end{array}$ & 46 \\
\hline Figure IV.13 & Rewritable data storage using rsEGFP & 47 \\
\hline Figure IV.14 & Histogram of the data spot fluorescence intensities & 48 \\
\hline Figure IV.15 & $\begin{array}{l}\text { RESOLFT microscopy of living E. coli cells } \\
\text { expressing rsEGFP-MreB }\end{array}$ \\
\hline Figure IV.16 & $\begin{array}{l}\text { RESOLFT microscopy of living PtK2 cells } \\
\text { expressing Keratin19-rsEGFP }\end{array}$ & 50 \\
\hline Figure IV.17 & $\begin{array}{l}\text { RESOLFT microscopy of living organotypic } \\
\text { hippocampal mice slice expressing Lifeact-rsEGFP }\end{array}$ & 51 \\
\hline
\end{tabular}


Figure IV.18 3D RESOLFT imaging of living E.coli cells 52 expressing rsEGFP-MreB

\begin{tabular}{|c|c|c|}
\hline Figure IV.19 & $\begin{array}{l}\text { Schematic of RESOLFT-type writing versus } \\
\text { diffraction limited writing }\end{array}$ & 53 \\
\hline Figure IV.20 & On-state specific bleaching of rsEGFP & 54 \\
\hline Figure IV.21 & $\begin{array}{l}\text { Subdiffractional writing using rsEGFP and visible } \\
\text { light }\end{array}$ & 54 \\
\hline Figure IV.22 & $\begin{array}{l}\text { Alignment of the amino acid sequences of EGFP, } \\
\text { rsEGFP and rsEGFP2 }\end{array}$ & 56 \\
\hline Figure IV.23 & Spectral properties of rsEGFP2 & 57 \\
\hline Figure IV.24 & Switching curves of rsEGFP2 & 58 \\
\hline Figure IV.25 & $\begin{array}{l}\text { Absorption spectra of equilibrium-state rsEGFP2 at } \\
\text { different } \mathrm{pH} \text { values }\end{array}$ & 58 \\
\hline Figure IV.26 & rsEGFP2 is a monomer & 59 \\
\hline Figure IV.27 & Maturation kinetic of rsEGFP2 at $37^{\circ} \mathrm{C}$ & 59 \\
\hline Figure IV.28 & $\begin{array}{l}\text { Expression of various functional rsEGFP2 fusion } \\
\text { proteins in mammalian PtK2 cells }\end{array}$ & 60 \\
\hline Figure IV.29 & $\begin{array}{l}\text { Comparison of off-switching halftimes of rsEGFP } \\
\text { and rsEGFP2 at different } 491 \mathrm{~nm} \text { light intensities }\end{array}$ & 60 \\
\hline Figure IV.30 & Switching fatigue of rsEGFP2 and rsEGFP & 61 \\
\hline Figure IV.31 & $\begin{array}{l}\text { RESOLFT imaging of Vimentin-rsEGFP2 in PtK2 } \\
\text { cells }\end{array}$ & 62 \\
\hline Figure IV.32 & $\begin{array}{l}\text { RESOLFT imaging of PtK2 cells ER using rsEGFP2 } \\
\text { as volume stain }\end{array}$ & 63 \\
\hline Figure IV.33 & RESOLFT time lapse imaging at 2 Hertz & 64 \\
\hline Figure IV.34 & $\begin{array}{l}\text { RESOLFT microscopy of living organotypic } \\
\text { hippocampal mice slice expressing lifeact-rsEGFP }\end{array}$ & 65 \\
\hline
\end{tabular}

\begin{tabular}{lll}
\hline Figure V.1 & Mutations of the rsEGFP & 71 \\
\hline
\end{tabular}

\begin{tabular}{llccc}
\hline Figure VI.1 & $\begin{array}{l}\text { Schematic overview of the } \\
\text { mutagenesis principle }\end{array}$ & site-directed & 91 \\
\hline Figure VI.2 & Scheme of the established RESOLFT & setup & 102 \\
\hline
\end{tabular}




\section{List of Tables}

\begin{tabular}{llr}
\hline Table IV.1 & $\begin{array}{l}\text { Foci diameter and maximum achievable light } \\
\text { intensities of the custom built screening setup }\end{array}$ & 37 \\
\hline Table IV.2 & Properties of EGFP, rsEGFP and Dronpa & 41 \\
\hline Table IV.3 & Propreties of EGFP, rsEGFP and rsEGFP2 & 57 \\
\hline & & 88 \\
\hline Table VI.1 & $\begin{array}{l}\text { The thermocycler protocol for standard PCR } \\
\text { amplification }\end{array}$ & 92 \\
\hline Table VI.2 & $\begin{array}{l}\text { The thermocycler protocol for site-directed } \\
\text { mutagenesis }\end{array}$ \\
\hline Table VI.3: & $\begin{array}{l}\text { The thermocycler protocol for error-prone } \\
\text { mutagenesis }\end{array}$ \\
\hline
\end{tabular}




\section{Acknowledgement}

In this chapter I would like to thank all the people who supported me during my work on this $\mathrm{PhD}$ thesis.

First I want to thank Prof. Stefan W. Hell for giving me the opportunity and the support to realize this project. Especially, I would like to thank my supervisor Prof. Stefan Jakobs. His advices, guidance and support were an inevitable help making this work possible. I also want to thank the thesis committee members Prof. Kai Tittmann and Dr. Dieter Klopfenstein for fruitful discussions and for giving new inspiration to my work.

Furthermore, I would like to thank my RESOLFT coworkers Ilaria Testa, Marcel Leutenegger, and Hannes Bock, for the great support and teamwork. Special thanks go to Christian Eggeling for his help and advices in all RESOLFT and physics related questions, as well as for his support in establishing the screening system. I also thank Nicolai Urban and Dr. Katrin Willig for providing and preparing the hypocampal brain slices of mice.

I am grateful to Chaitanya Ullal and Jaydev Jethwa for proofreading of the manuscript.

I would also like to thank Sylvia Löbermann, Rita Schmitz-Salue, Tanja Gilat, Ellen Rothermel, and Maria Sermond for helping and supporting me in the lab. Especially, I would like to thank the people of the RSFP-group Dr. Tanja Brakemann, Dr. Martin Andresen, Dr. André C. Stiel, and Dr. Nickels Jensen for the support, for the fruitful discussions, for motivating me and most of all for the great time, which I would not want to miss. Furthermore, I would like to thank Dr. Christian Wurm, Daniel Jans, Dr. Stefan Stoldt, Dr. Daniel Neumann, Marlen Stäglich, Franziska Stagge, Lena Große, Dr. Susann Kummer, Dr. Peter Ilgen, Sebastian Berning, Dr. Flavie Lavoie-Cardinal and all other former and present members of the Mitochondrial Structures and Dynamics Group and the Department for NanoBiophotonics for the wonderful working atmosphere.

My special thanks go to my family, especially my parents and Claudia. This work would not have been possible without their help, support, patience, encouragement, understanding and motivation. 


\section{Publications}

Grotjohann, T.*, Testa, I.*, Leutenegger, M.*, Bock, H., Urban, N.T., LavoieCardinal, F., Willig, K.I., Eggeling, C., Jakobs, S. and Hell, S.W. (2011). "Diffraction-unlimited all-optical imaging and writing with a photochromic GFP." Nature 478(7368): 204-208.

Brakemann, T.*, Stiel, A.C. ${ }^{*}$ Weber, G., Andresen, M., Testa, I., Grotjohann, T., Leutenegger, M., Plessmann, U., Urlaub, H., Eggeling, C., Wahl, M.C., Hell, S.W. and Jakobs, S. (2011). "A reversibly photoswitchable GFP-like protein with fluorescence excitation decoupled from switching." Nat Biotechnol 29(10):942947.

* these authors contributed equally to this work 


\section{Curriculum vitae}

Tim Grotjohann

date of birth:

born in: $10^{\text {th }}$ February, 1981

Hannover adress

Südring 38

37120 Bovenden

Germany

phone no.: 0551/2006444

email: tgrotjo@gwdg.de

\section{education:}

Since 2008

Feb. 2008

2001-2008

2000-2001

Jul. 2000

1987-2000

PhD thesis:

"Generation of Novel Photochromic GFPs: Fluorescent Probes for RESOLFT-type Microscopy at Low Light Intensities"

Georg-August-University Göttingen, Germany prepared at the Max-Planck-Institute for Biophysical Chemistry, Germany, Department of NanoBiophotonics, Mitochondrial Structure and Dynamics Group

Diploma (sehr gut)

"Etablierung von fluoreszenzmikroskopischen "High Content Screening"-Systemen zur Identifikation von pharmakologisch aktiven Substanzen in Insektenzellen“

University of Bielefeld, Germany

Diploma studies in biology

University of Bielefeld, Gemany

alternative civilian service

at the Ökologische Schutzstation Steinhuder Meer e.V.

Abitur at the Hölty-Gymnasium Wunstorf, Germany

schooling:

primary school: Grundschule Kolenfeld

5. - 6. class at: Orientierungsstufe Süd Wunstorf

secondary school: Hölty-Gymnasium Wunstorf 